\title{
33. GEOCHEMISTRY OF BASALTS FROM THE SHIKOKU AND DAITO BASINS, DEEP SEA DRILLING PROJECT LEG 58
}

\author{
Nicholas G. Marsh, Andrew D. Saunders, ${ }^{1}$ and John Tarney, Department of Geological Sciences, \\ University of Birmingham, U.K. \\ and \\ Henry J. B. Dick, Department of Geology and Geophysics, Woods Hole Oceanographic Institution, \\ Woods Hole, Massachusetts
}

\begin{abstract}
Leg 58 successfully recovered basalt at Sites 442,443 , and 444 , in the Shikoku Basin, and at Site 446 in the Daito Basin. Only at Site 442 did penetration reach unequivocal oceanic layer 2; at the other sites, only off-axis sills and flows were sampled. Petrographic observations indicate that back-arc basalts from the Shikoku Basin, with the exception of the kaersutite-bearing upper sill at Site 444 , are mineralogically similar to basalts being erupted at normal mid-ocean ridges. However, the Shikoku Basin basalts are commonly very vesicular, indicating a high volatile content in the magmas. Site 446 in the Daito Basin penetrated a succession of 23 sills which include both kaersutite-bearing and kaersutite-free basalt varieties.

A total of 187 samples from the four sites has been analyzed for major and trace elements using X-ray-fluorescence techniques. Chemically, the basalts from Sites 442 and 443 and the lower sill of Site 444 are subalkaline tholeiites and resemble $\mathrm{N}$-type ocean-ridge basalts found along the East Pacific Rise and at $22^{\circ} \mathrm{N}$ on the Mid-Atlantic Ridge (MAR), although they are not quite as depleted in certain hygromagmatophile (HYG) elements. They do not show any chemical affinities with island-arc tholeiites. The basalts from Site 446 and from the upper sill at Site 444 show alkaline and tholeiitic tendencies, and are enriched in the more-HYG elements; they chemically resemble enriched or E-type basalts and their differentiates found along sections of the MAR (e.g., $45^{\circ} \mathrm{N}$ ) and on ocean islands (e.g., Iceland and the Azores).

Most of the intra-site variation may be attributed to crystal settling within individual massive flows and sills, to high-level fractional crystallization in sub-ridge magma chambers, or, where there is evidence of a long period of magmatic quiescence between units, to batch partial melting. However, the basalts from Sites 442 and 443 and from the lower sill at Site 444 cannot easily be related to those from Site 446 and the upper sill at Site 444 , and it is possible that the different basalt types were derived from chemically distinct mantle sources. From comparison of the Leg 58 data with those already available for other intra-oceanic back-arc basins, it appears that the mantle sources giving rise to back-arc-basin basalts are chemically as diverse as those for mid-ocean ridges. In addition, the high vesicularity of the Shikoku Basin basalts supports previous observations that the mantle source of back-arc-basin basalts may be contaminated by a hydrous component from the adjacent subduction zone.
\end{abstract}

\section{INTRODUCTION}

During Leg 58, basaltic rocks were recovered at four of the five sites occupied in the Shikoku and Daito Basins. At Sites 442,443 , and 444 , in the intra-oceanic

\footnotetext{
${ }^{1}$ Present address: Department of Geology, Bedford College, University of London.
}

Shikoku back-arc or marginal basin, lying between the Iwo Jima and Kyushu-Palau Ridges, sampled basalts are no older than 21,17 , and 15 m.y., respectively, from both sides of the north-south-trending axial zone. Only at Site 442 were basalts penetrated which belong unequivocally to oceanic layer 2; at Sites 443 and 444, recovered basalts probably represent off-axis intrusive or extrusive activity. At Site 446, in the Daito Basin, sills of post-early Eocene age were penetrated, but basement 
was not reached; in this region it is thought to be oceanic (Karig, 1975).

It has been established by detailed geophysical investigation and by dredging operations that most back-arc or marginal basins are of extensional origin, and are floored by basaltic crust broadly comparable in structure and composition to that of the major ocean basins (Karig, 1971, 1974; Hart et al., 1972; Barker, 1972; Hawkins, 1974; Gill, 1976). However, there is still no consensus as to whether all marginal-basin basalts are encompassed by the range of basalt compositions presently being erupted at mid-ocean ridges, or whether they have geochemical characteristics which are, to a small degree, transitional towards orogenic basalts, as indicated by detailed chemical investigations of lavas from back-arc centers in the East Scotia Sea (Saunders and Tarney, 1979) and Bransfield Strait (Weaver et al., 1979). Similar studies of basalts from the Mariana Trough (Hart et al., 1972) and Lau Basin (Hawkins, 1976, 1977), however, do not corroborate the evidence from the Scotia Sea and Bransfield Strait basins. It is important, therefore, to assess the compositions of magmas being erupted in several back-arc basins before any general conclusions about their genesis can be made. The prominent role of back-arc basins in the formation of many ophiolites (Dewey, 1976; Saunders et al., 1979) emphasizes the importance of being able to chemically characterize and distinguish back-arc-basin basalts from ocean-ridge basalts, and this can be achieved only when sufficient data on modern back-arc-basin basalts are available.

Comprehensive studies of basalts from the North Atlantic Ocean have illustrated the chemical variability of magmas being erupted along the Mid-Atlantic Ridge (Schilling, 1973, 1975a,b; Hart et al., 1973; White et al., 1976; Bougault et al., 1979; Tarney et al., 1978, 1979, in press; Wood et al., 1979). It is therefore important, before any detailed comparisons between back-arc-basin and ocean-ridge basalts are made, that the composition of ocean-ridge basalts be clearly delineated. Sun et al. (1979) and Tarney et al. (in press) have defined three main basalt types presently being erupted in the North Atlantic: normal, or N-type, depleted ocean-ridge basalts; enriched, or E-type, basalts; and T-type basalts, which have a composition transitional between $\mathrm{N}$ - and E-type basalts. These terms will be defined in detail later. The variations in chemistry appear to be in part due to chemical heterogeneities in the mantle source, in addition to variations caused by partial melting and fractional crystallization (see discussions by Bougault et al., 1979; O'Nions et al., 1976; Tarney et al., 1978, 1979, in press; Wood et al., 1979). It is necessary to assess whether or not back-arc-basin basalts exhibit a similar range in chemistry.

Leg 58 has recovered basaltic material which affords an excellent opportunity to undertake detailed petrological and geochemical studies of basalts from a "typical" Western Pacific intra-oceanic back-arc basin. In this paper we shall be concerned essentially with X-rayfluorescence data on major and trace elements. Basalts from Leg 49 in the North Atlantic were analyzed using the same analytical equipment (Tarney et al., 1978), allowing regional comparisons without inter-laboratory error. Instrumental conditions were arranged to provide the highest precision possible, especially for the trace elements. Thus, reported geochemical differences, both within and between sites, are significant. However, we have confined ourselves to an essentially qualitative assessment of the results.

\section{REGIONAL AND TECTONIC SETTING}

\section{The Shikoku Basin}

The Shikoku Basin (Figure 1) has the form of an elongate trough some $1200 \mathrm{~km}$ long, $500 \mathrm{~km}$ wide, and $4.5 \mathrm{~km}$ deep, bounded by the Iwo Jima Ridge to the east and by the Kyushu-Palau Ridge to the west. The northern end of the basin is delineated by the Nankai Trough and by the Island of Shikoku. Southward, the basin continues without any physical barrier into the Parece Vela Basin.

The Shikoku Basin has been identified as an inter-arc basin separating a composite frontal arc system - comprising the Iwo Jima Ridge, Bonin Trough, and Bonin Ridge - from the remnant Kyushu-Palau Ridge. Subduction of Pacific Ocean floor beneath the Bonin Ridge is presently occurring along the Izu-Bonin Trench, and it is apparent that the Bonin Trough is an incipient back-arc basin (Karig, 1971). The Nankai Trough probably represents the site of subduction of Shikoku Basin floor beneath southern Japan (Karig, Ingle, et al., 1975; Kobayashi and Isezaki, 1976).

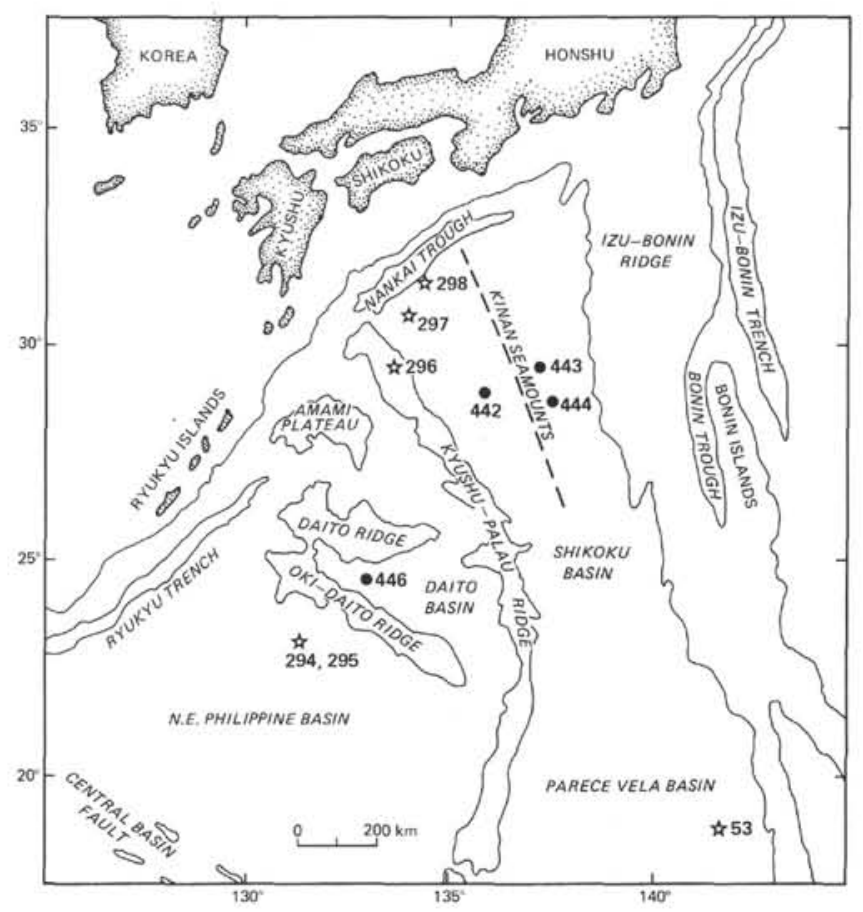

Figure 1. Map of the northern Philippine Sea, showing the location of Leg 58 sites in the Shikoku and Daito Basins. 
Geophysical studies have shown that the Shikoku Basin is floored by oceanic crust, although the absence of detectable shallow earthquakes beneath the axial zone of the basin (Katsumata and Sykes, 1969) suggests that spreading is not now taking place. However, the axial zone has the high heat flow and topography characteristic of many presently active oceanic spreading centers (Kobayashi and Isezaki, 1976), although the magnetic lineations observed within the basin are of lower amplitude than those found in most major ocean basins. Linear, weak magnetic anomalies running parallel to the Kyushu-Palau Ridge are well established for the western side of the basin, but because of rough topography the interpretation of anomalies in the eastern side of the basin remains equivocal (Watts and Weissel, 1975; Kobayashi and Isezaki, 1976; Shih, this volume). Available data suggest that spreading in the Shikoku Basin began between 25 and $27 \mathrm{Ma}$ and ceased about $17 \mathrm{Ma}$ (Watts and Weissel, 1975; Kobayashi and Isezaki, 1976). This effectively separated the continental crustal slivers of the Kyushu-Palau and Iwo Jima Ridges (Murauchi et al., 1976). Alternative interpretations of the data are suggested by Tomada et al. (1975), who consider that the spreading occurred later, between 22 and $10 \mathrm{Ma}$, and by Murauchi et al. (1976), who have proposed that spreading occurred in two distinct episodes, 24 to $19 \mathrm{Ma}$ and 12 to $16 \mathrm{Ma}$. The interpretations agree, however, in suggesting that spreading is not now occurring in the Shikoku Basin. It would appear that the locus of spreading has migrated westward into what is now the Bonin Trough.

\section{Northeast Philippine Basin}

The northeast corner of the Philippine Basin is roughly triangular; it is bordered by the Ryukyu Trench to the northwest and by the Kyushu-Palau Ridge to the east (Figure 1). The area comprises a complex group of basins and topographic highs, including the Oki-Daito and Daito Ridges, which apparently are structurally similar to island arcs (Murauchi et al., 1968). The intervening basins (e.g., Daito Basin) are sufficiently deep to be floored by old ocean-crust, possibly of early-Tertiary or late-Cretaceous age (Karig, 1975). However, a thick pile of sediments now obscures the oceanic layer 2, although heat-flow measurements (Watanabe et al., 1970) show values similar to those of old ocean crust.

Rocks previously dredged from the Daito Ridge include greenschists, hornblende schists, serpentinites, andesite, and diorite. Andesite, basalt, and granodiorite have been recovered from the Oki-Daito Ridge. From the geophysical evidence and dredge hauls it has been suggested that the Daito and Oki-Daito Ridges formed as island arcs (Murauchi et al., 1968; Karig, 1975; Mizuno et al., 1975, in press). These arcs and the adjoining pieces of ocean crust drifted some $2000 \mathrm{~km}$ northward during the last $52 \mathrm{~m}$.y. as a result of sea-floor spreading in the West Philippine Sea, before being trapped against the Kyushu-Palau Ridge.

\section{ANALYTICAL TECHNIQUES}

\section{Sample Preparation}

Minicores or quarter-core sections were washed in distilled water to remove surface contamination, and dried at $100^{\circ} \mathrm{C}$ prior to being ground to a fine powder (less than $60 \mu \mathrm{m}$ ) in the shipboard tungsten-carbide TEMA swing mill. Powder briquettes $46 \mathrm{~mm}$ in diameter were made by binding 15 grams of rock powder with about 30 drops of a 7 per cent aqueous solution of polyvinyl alcohol, under a pressure of 15 tons against the polished, hardened faces of a steel die.

Representative samples from Sites 442, 443, and 444, and all the samples from Site 446, were made into fusion beads for the purpose of major-element analysis. This technique eliminates mineralogical effects and reduces matrix absorption. The rock powders were dried overnight at $110^{\circ} \mathrm{C}$, and ignition loss was determined by heating at $1000^{\circ} \mathrm{C}$ in an electric furnace for 1 hour. Beads were prepared by mixing sufficient rock powder and flux in a $\mathrm{Pt} / \mathrm{Au}$ crucible to give an exact ratio of 1:5 after fusion ( 0.9 gram rock, 4.5 gram flux, ignited material). Fusion was carried out at $1200^{\circ} \mathrm{C}$, with frequent swirling, for 20 minutes in a vertical-tube electric furnace. The flux, 80 per cent $\mathrm{Li}$ metaborate, 20 per cent $\mathrm{Li}$ tetraborate (Johnson Matthey Spectroflux 100B), is described by Bennett and Oliver (1976) and is designed to dissolve the widest possible range of silicate and ceramic materials.

After fusion, the melt was pressed in aluminum molds, using equipment similar to that described by Harvey et al. (1973); the beads were transferred to heated insulating blocks for annealing at $200^{\circ} \mathrm{C}$ for 30 minutes, and finally allowed to cool in the blocks. The lithium borate beads are slightly hygroscopic and must be stored in sealed polypropylene bags in a desiccator to prevent atmospheric attack of the bead surface.

\section{X-Ray-Fluorescence Analysis}

The equipment used was a Philips PW1450 automatic spectrometer with a PW1466 60-position sample changer. Count data from the spectrometer were processed using a 32K Digital PDP11-03 computer, and the University of Birmingham ICL1906A computer. Trace elements were determined on 46-mm powder briquettes, using molybdenum ( $\mathrm{Y}, \mathrm{Sr}, \mathrm{Rb}, \mathrm{Th}, \mathrm{Pb}, \mathrm{Ga}, \mathrm{Zn}, \mathrm{Ba})$ and tungsten $(\mathrm{Ni}, \mathrm{Cr}, \mathrm{Ce}, \mathrm{La}, \mathrm{Zr}, \mathrm{Nb})$ anode $\mathrm{X}$-ray tubes. Major- and minor-element analyses of the basalts from Sites 442,443 , and 444 were determined on $46-\mathrm{mm}$ powder briquettes and representative fusion beads using a chromium-anode X-ray tube. Major- and minor-element analyses of basalts from Site 446 were determined on fusion beads, using a rhodium-anode X-ray tube. Instrumental repeatability was ensured by ratioing counts to a reference sample, re-analyzing every fourth sample ( $\mathrm{Rh}$ and $\mathrm{Cr}$ tubes) or at the beginning of every loading of 60 samples (Mo and W tubes). 


\section{Instrumental Conditions}

Details of instrumental conditions for analysis of trace elements are given in Tarney et al. (1978).

The Rh-anode X-ray tube, operated at $30 \mathrm{kV}, 70 \mathrm{~mA}$, provides equivalent or superior sensitivity for those elements previously analyzed using a $\mathrm{Cr}$ anode, with the exception of $\mathrm{Ti}, \mathrm{Ca}$, and $\mathrm{K}$. However, these elements are extremely sensitive to $\mathrm{Cr}$ excitation; it is usually necessary to greatly reduce tube power to avoid saturating the detector, and therefore no problems are encountered with the reduced efficiency of the Rh anode. An additional advantage is that the analysis of $\mathrm{Mn}$ is possible without filtering out the $\mathrm{Cr} \mathrm{K}_{\beta}$ radiation from the tube target.

The $K_{\alpha}$ wavelengths of all major elements were analyzed using the flow-proportional detector (argon/methane) with automatic pulse-height selection. The fine collimator $(0.15 \mathrm{~mm})$ was used for all elements except $\mathrm{Na}$ and $\mathrm{Al}$, for which the coarse collimator $(0.55 \mathrm{~mm})$ was employed. The crystals used were: $\operatorname{LiF}_{200}(\mathrm{Mn}), \mathrm{LiF}_{220}$ (Fe), PET ( $\mathrm{Ti}, \mathrm{Ca}, \mathrm{K}, \mathrm{Si}, \mathrm{Al})$, TlAP (Mg, Na), and $\mathrm{Ge}_{111}$ (P). The germanium crystal was chosen for $\mathrm{P}$ to avoid the overlap of the escape peak arising from the secondorder $\mathrm{CaK}_{\beta}$ on $\mathrm{PK}_{\alpha}$. A symmetrical, 50 per cent width window was used for the pulse-height discriminator for all elements, with the exception of $\mathrm{Mg}$, for which a setting of 20 to 65 per cent is needed to reduce the overlap of the third-order $\mathrm{CaK}_{\alpha}$. However, both these $\mathrm{Ca}$ interferences are considerably less than when using $\mathrm{Cr}$ excitation.

\section{Calibration}

Initial trace-element calibrations were prepared from a wide range of rock types spiked with an appropriate pure-element compound (Leake, Hendry, et al., 1969). These values were then related using either the $\mathrm{WL}_{\beta}$ or $\mathrm{MoK}_{\alpha}$ Compton scatter lines to provide correction for total mass absorption for all emission lines shorter than the iron absorption edge (Reynolds, 1967). Ce and La calibrations were prepared using basalts previously analyzed by isotope dilution. The accuracy of the various calibration lines was monitored by analyzing several international reference samples of suitable compositions.

For the major elements, primary calibrations and " $\alpha$ " (matrix-dependent constant) correction factors were calculated from binary and ternary mixtures of appropriate pure chemicals and referred to an average basalt composition. The validity of these calibrations was checked against departmentally analyzed material and international reference standards. Blank values for minor elements were checked using pure $\mathrm{SiO}_{2}, \mathrm{Al}_{2} \mathrm{O}_{3}$, and $\mathrm{BeO}$, and were found to be low but significant for $\mathrm{Na}_{2} \mathrm{O}, \mathrm{K}_{2} \mathrm{O}$, and $\mathrm{P}_{2} \mathrm{O}_{5}$. For these elements at very low levels the values produced from undiluted powder briquettes are preferred.

\section{Precision and Accuracy}

The precision of measurement and sample preparation is illustrated by the standard deviation of six separate beads of the internal ocean basalt reference sample BOB-1 (Table 1). The trace-element data in Table 1 were determined on powder briquettes. The instrumental precision with the techniques used is high, even at relatively low trace-element concentrations. However, a potential problem of instrumental analysis is long-term drift when periods greater than 12 months are considered. This has been kept to a minimum by using 30 samples from Leg 49 (Tarney et al., 1979) together with other basaltic material, selected to give as wide a concentration range as possible for all elements. These samples are analyzed together with the unknowns, ensuring compatibility of data between different legs. For major-element analysis it is not desirable to reanalyze fusion beads over long periods of time, hence a number of internal reference samples are fused and analysed with each batch of unknowns.

Accuracy of analysis can only be judged against international reference samples which, for trace elements, do not cover the range of concentrations encountered in most DSDP legs. Data for trace elements in basalt

TABLE 1

Analyses of Standard Reference Samples ${ }^{\mathrm{a}}$

\begin{tabular}{|c|c|c|c|}
\hline \multirow{2}{*}{$\begin{array}{l}\text { Component } \\
\text { (\% or ppm) }\end{array}$} & \multicolumn{3}{|c|}{ Standard } \\
\hline & BOB-1 & AVG-1 & JB-1 \\
\hline $\mathrm{SiO}_{2}$ & $51.02 \pm 0.078$ & 60.19 & 53.13 \\
\hline $\mathrm{TiO}_{2}$ & $1.28 \pm 0.004$ & 1.01 & 1.30 \\
\hline $\mathrm{Al}_{2} \mathrm{O}_{3}$ & $16.55 \pm 0.012$ & 17.44 & 14.48 \\
\hline $\mathrm{Fe}_{2} \mathrm{O}_{3}$ & $8.48 \pm 0.013$ & 6.98 & 9.02 \\
\hline $\mathrm{MnO}$ & $0.14 \pm 0.001$ & 0.10 & 0.15 \\
\hline $\mathrm{MgO}$ & $7.58 \pm 0.030$ & 1.56 & 7.79 \\
\hline $\mathrm{CaO}$ & $11.39 \pm 0.027$ & 4.87 & 9.41 \\
\hline $\mathrm{Na}_{2} \mathrm{O}$ & $3.10 \pm 0.074$ & 4.40 & 2.87 \\
\hline $\mathrm{K}_{2} \mathrm{O}$ & $0.37 \pm 0.003$ & 2.99 & 1.45 \\
\hline $\mathrm{P}_{2} \mathrm{O}_{5}$ & $0.16 \pm 0.001$ & 0.49 & 0.25 \\
\hline \multirow[t]{2}{*}{ Total } & 100.07 & 100.03 & 99.85 \\
\hline & BOB-1 & BCR-1 & JB-1 \\
\hline $\mathrm{Ni}$ & $106.4 \pm 2.1$ & 19 & 134 \\
\hline $\mathrm{Cr}^{\mathrm{b}}$ & $257 \pm 10$ & 18 & 359 \\
\hline $\mathrm{Rb}$ & $4.8 \pm 0.6$ & 48 & 42 \\
\hline $\mathrm{Sr}$ & $202.1 \pm 1.0$ & 329 & 442 \\
\hline $\mathrm{Y}$ & $26.9 \pm 0.5$ & 36 & 23 \\
\hline $\mathrm{Zr} \mathrm{c}$ & $107.6 \pm 3.0$ & 191 & 150 \\
\hline $\mathrm{Nb}$ & $5.3 \pm 1.6$ & 12 & 35 \\
\hline $\mathrm{Ba}^{\mathrm{d}}$ & $50.4 \pm 3.6$ & 695 & 500 \\
\hline $\mathrm{La}$ & $6.4 \pm 1.9$ & 26 & 38 \\
\hline $\mathrm{Ce}$ & $16.6 \pm 1.7$ & 55 & 66 \\
\hline $\mathrm{Zn}$ & $61.5 \pm 2.1$ & 120 & 80 \\
\hline Ga & $17.6 \pm 0.7$ & 25 & 17 \\
\hline
\end{tabular}

a $\mathrm{Fe}_{2} \mathrm{O}_{3}$ is total iron as $\mathrm{Fe}_{2} \mathrm{O}_{3}$; mean and 1 sigma standard deviation of six fusion beads of BOB-1, and means of three fusion beads of international reference samples; all values are for ignited samples; average concentration of $\mathrm{BOB}-1$ determined on 22 consecutive days (with 1 sigma variation); average concentration of two samples of international reference material.

b Not corrected for $\mathrm{V}$ interference.

cCorrected for $\mathrm{Sr}$ interference.

dCorrected for Ce interference. 
BCR-1 are given in Tarney et al. (1979) and for a wider range of materials in Hendry (1975). Major elements for AGV-1 and JB-1 are given in Table 1.

\section{SHIKOKU BASIN}

Three sites, comprising a total of six holes, were drilled in the Shikoku Basin during Leg 58. The igneous stratigraphy of the recovered sections from each of these sites is given in Figures 2, 17, and 27 in this paper, and although detailed petrographic descriptions are given in the relevant site chapters (this volume), brief resumes are included in the following sections.

\section{Site $\mathbf{4 4 2}$}

Site 442 (Figure 1) is approximately $50 \mathrm{~km}$ west of the axial zone of the Shikoku Basin, at $28^{\circ} 59.00^{\prime} \mathrm{N}, 136^{\circ}$ 03.43 'E, on magnetic anomaly 6 (Kobayashi and Nakata, 1977; 19-20 m.y.: LaBrecque et al., 1977). Three holes were drilled; Hole 442 was a test hole for the reentry cone, and Holes $442 \mathrm{~A}$ and $442 \mathrm{~B}$ cored approximately 158 meters of massive basalt flows and pillow basalts beneath some 300 meters of sediment, at a water depth of about 4635 meters.

Site 442 is the only site of Leg 58 from which pillow basalts typical of oceanic Layer 2 were definitely recovered. Two lithologic units have been recognized: Unit 1, comprising mainly massive flows or sills, and unit 2, the underlying pillow lava sequence (Figure 2). The basalts from both units are vesicular and sparsely olivine bearing. Unit 1 is separated from unit 2 by approximately 2 meters of brown mudstone, for which microfossils indicate an age between 18 and $21 \mathrm{~m} . \mathrm{y}$. This is in agreement with the magnetic-anomaly age
(19-20 m.y.) of the basement at this site. The oldest sediments overlying unit 1 are limestones containing microfossils giving an age between 15 and 17 m.y.

Unit 1 consists of approximately 59 meters of fine- to medium-grained basalt, which has been divided into 16 sub-units. Many of these sub-units have fine-grained or occasionally aphanitic chill zones, and the absence of glassy margins suggests that these basalts were emplaced as sills or as massive flows (Site 442 report, this volume). However, the presence of glassy selvages and variolitic basalts in Core 6 of Hole 442B indicates that the unit may consist, at least in part, of pillow lavas.

The sub-units of unit 1 grade from fine-grained basalts to medium-grained dolerites with an inequigranular, subophitic texture. Seriate textures are common. In the finer-grained samples, intersertal to intergranular augite and plagioclase $\left(\mathrm{An}_{55-75}\right)$ and interstitial magnetite are embedded in a cryptocrystalline groundmass which is often replaced by clays. Strongly zoned clinopyroxene crystals, colored green in their centers to brown at the edges, and "bow-tie" zoned clinopyroxenes are present in the coarsest-grained sections of some sub-units. In these coarse-grained rocks, it is difficult to ascertain whether such crystals are phenocrysts or merely large groundmass crystals. However, they frequently form glomerocrystic intergrowths with zoned plagioclase crystals (core compositions of $\mathrm{An}_{70-85}$ ). Chrome spinel is a common accessory phase throughout unit 1 , generally appearing as inclusions in plagioclase phenocrysts. Chrome-spinel inclusions also occur in clinopyroxene phenocrysts. The basalts and dolerites of unit 1 tend to be very vesicular, often with two distinct populations of vesicles. Most sub-units have 10 to 30 per cent small vesicles $(<1 \mathrm{~mm})$ and generally 1 to 5 per cent

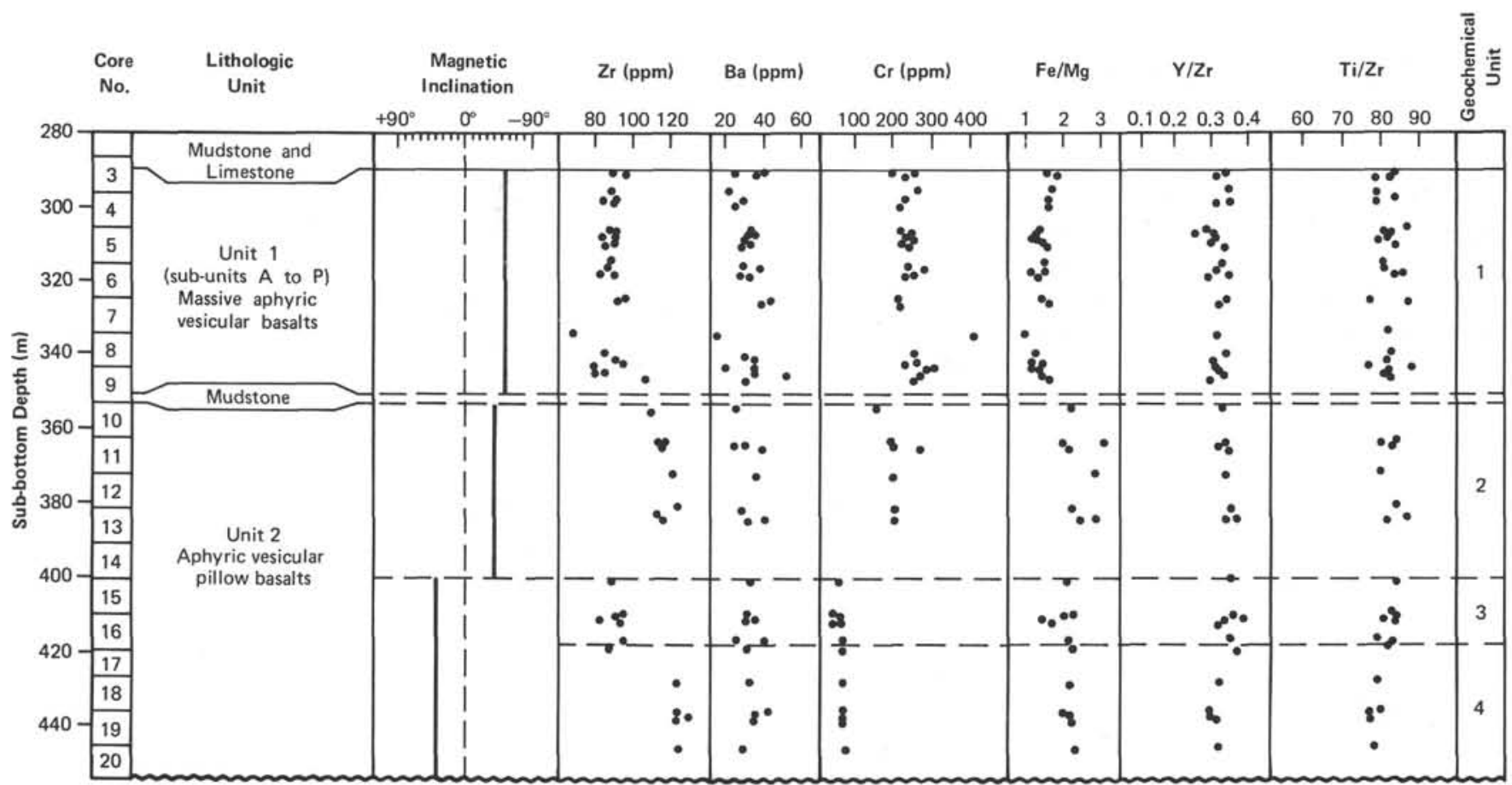

Figure 2. Down-hole variation in lithology, magnetic inclination, and selected geochemical parameters at Site 442. 
large vesicles $(2-5 \mathrm{~mm})$. Pipe vesicles are also found near the chill margins of several sub-units.

The pillow basalts of unit 2 have glassy margins, generally less than $1 \mathrm{~cm}$ thick, and contain 1 to 2 per cent small plagioclase laths at their inner margins. The glassy zones grade abruptly into variolitic zones toward the pillow interiors. The varioles consist of small anhedral plagioclase laths and clinopyroxene microlites in a glassy matrix. The cores of the pillows are usually fine grained, with an intersertal texture comprising 25 per cent plagioclase, $\left(\mathrm{An}_{55-70}\right), 30$ per cent augite, 2 to 3 per cent granular magnetite, and a glassy or (more commonly) a clay-rich matrix. Vesicles are again common, composing up to 40 per cent of the rock, and are usually less than $1 \mathrm{~mm}$ across. However, larger vesicles up to 10 $\mathrm{mm}$ across do occur, and pipe vesicles are common, usually in a zone between 1 and $10 \mathrm{~cm}$ from the glassy margin.

Low-temperature alteration is a general feature of all the basalts recovered at this site. The cryptocrystalline or glassy matrixes are commonly replaced by clay minerals. The basalts are commonly cut by calcite- and claylined fractures or veins, and the vesicles are generally filled or lined by similar material. Zeolites are rare, and pyrite was found in small amounts in vesicles and fractures of sub-unit $1 \mathrm{~K}$ only, which also contained some chlorite and talc-like alteration products (Site 442 report, this volume).

Major- and trace-element data for the basalts recovered at Site 442 are listed in Tables 2 and 3. In the following discussion, emphasis will be placed on the basalts recovered from Hole 442B, the range of compositions of which generally encompass those from Hole 442A. The basalts have been grouped in different geochemical units according to variations in their minor- and trace-element contents, as illustrated in Figure 2 . These variations broadly coincide with the lithologic and magnetic units, although lithologic unit 2 (Hole 442B) has been subdivided into three geochemical units. The geochemical units and the lithologic units do not necessarily coincide.

Site 442 basalts are hypersthene and olivine normative (subalkaline), with practically no normative nepheline or quartz (Figure 3); the minor transgressions into the $\mathrm{Ne}$ and Q fields on Figure 3 may be due to the presence of secondary carbonate and clays, respectively. The basalts also have a high normative-plagioclase content, even though plagioclase phenocrysts are not a major phase, and this is shown in Figure 4, where the basalts plot as plagioclase tholeiites on the pyroxene-plagioclase-olivine field of Shido et al. (1971). Geochemical unit 442-1 is generally more olivine normative than the underlying pillow lavas (units $442-2$ to $442-4$ ), and this is also reflected in the lower $\mathrm{Fe} / \mathrm{Mg}$ ratios, and higher $\mathrm{Cr}$ and $\mathrm{Ni}$ abundances in Unit 442-1 (Figures 5 and 6).

There is considerable downhole variation in absolute element abundances at Site 442. The essential features of this variation are illustrated in Figure 2. There is a clear correlation between units based on magnetic inclination and geochemistry, although geochemical unit
TABLE 2

Analyses, Element Ratios, and CIPW Norms for Basalts from Hole $442 \mathrm{~A}^{\mathrm{a}}$

\begin{tabular}{|c|c|c|c|c|c|c|c|c|}
\hline $\begin{array}{l}\text { Core } \\
\text { Section } \\
\text { Interval (cm) }\end{array}$ & $\begin{array}{r}442 \mathrm{~A}-31 \\
1 \\
85\end{array}$ & $\begin{array}{r}32 \\
2\end{array}$ & $\begin{array}{r}32 \\
2 \\
80\end{array}$ & $\begin{array}{r}33 \\
1 \\
124\end{array}$ & $\begin{array}{r}33 \\
2 \\
57\end{array}$ & $\begin{array}{r}33 \\
3 \\
68\end{array}$ & $\begin{array}{r}33 \\
4 \\
111\end{array}$ & $\begin{array}{r}34 \\
1 \\
78\end{array}$ \\
\hline $\mathrm{SiO}_{2}(\%)$ & 49.6 & 48.6 & 49.4 & 47.5 & 49.9 & 49.8 & 49.9 & 49.8 \\
\hline $\mathrm{TiO}_{2}$ & 1.24 & 1.12 & 1.16 & 1.09 & 1.23 & 1.22 & 1.20 & 1.15 \\
\hline $\mathrm{Al}_{2} \mathrm{O}_{3}$ & 16.2 & 15.8 & 16.0 & 15.4 & 15.9 & 15.8 & 15.2 & 16.1 \\
\hline $\mathrm{Fe}_{2} \mathrm{O}_{3}$ & 8.66 & 8.38 & 7.29 & 7.96 & 9.66 & 9.13 & 10.09 & 8.62 \\
\hline $\mathrm{MnO}^{\circ}$ & 0.14 & 0.14 & 0.10 & 0.14 & 0.13 & 0.12 & 0.13 & 0.11 \\
\hline $\mathrm{MgO}$ & 6.12 & 5.38 & 6.77 & 6.16 & 6.53 & 7.17 & 6.55 & 7.04 \\
\hline $\mathrm{CaO}$ & 13.46 & 15.19 & 13.79 & 16.71 & 11.47 & 11.60 & 11.74 & 12.37 \\
\hline $\mathrm{Na}_{2} \mathrm{O}$ & 2.70 & 2.67 & 2.78 & 2.40 & 2.77 & 2.82 & 2.84 & 2.72 \\
\hline $\mathrm{K}_{2} \mathrm{O}$ & 0.26 & 0.40 & 0.25 & 0.24 & 0.38 & 0.33 & 0.52 & 0.23 \\
\hline $\mathrm{P}_{2} \mathrm{O}_{5}$ & 0.18 & 0.16 & 0.15 & 0.16 & 0.15 & 0.14 & 0.14 & 0.14 \\
\hline Total & 98.55 & 97.83 & 97.72 & 97.77 & 98.13 & 98.20 & 98.32 & 98.30 \\
\hline Ni (ppm) & 45 & 57 & 130 & 53 & 49 & 77 & 35 & 64 \\
\hline $\mathrm{Cr}$ & 220 & 234 & 240 & 216 & 233 & 245 & 214 & 245 \\
\hline $\mathrm{Zn}$ & 78 & 63 & 69 & 116 & 63 & 66 & 65 & 68 \\
\hline Ga & 17 & 17 & 17 & 16 & 18 & 18 & 19 & 18 \\
\hline $\mathrm{Rb}$ & 4 & 9 & 4 & 4 & 7 & 6 & 26 & 2 \\
\hline Sr & 186 & 183 & 182 & 186 & 189 & 182 & 178 & 186 \\
\hline $\mathrm{Y}$ & 33 & 28 & 26 & 28 & 29 & 26 & 27 & 27 \\
\hline $\mathrm{Zr}$ & 84 & 86 & 86 & 82 & 86 & 89 & 88 & 87 \\
\hline $\mathrm{Nb}$ & 3 & 4 & $<1$ & 3 & 1 & 3 & 3 & 3 \\
\hline $\mathrm{Ba}$ & 37 & 28 & 29 & 21 & 27 & 30 & 31 & 22 \\
\hline $\mathrm{La}$ & 4 & 2 & 4 & 3 & 6 & 3 & 5 & 5 \\
\hline $\mathrm{Ce}$ & 10 & 11 & 13 & 13 & 19 & 13 & 12 & 11 \\
\hline $\mathrm{Pb}$ & 5 & 3 & 5 & 7 & $<1$ & 3 & 3 & 2 \\
\hline Th & $<1$ & $<1$ & 1 & $<1$ & $<1$ & $<1$ & $<1$ & $<1$ \\
\hline $\mathrm{Zr} / \mathrm{Nb}$ & 28 & 21 & - & 27 & 86 & 30 & 29 & 29 \\
\hline $\mathrm{Ti} / \mathrm{Zr}$ & 89 & 78 & 81 & 80 & 86 & 82 & 82 & 79 \\
\hline $\mathrm{Y} / \mathrm{Zt}$ & 0.39 & 0.33 & 0.30 & 0.34 & 0.34 & 0.29 & 0.31 & 0.31 \\
\hline $\mathrm{Ce} / \mathrm{Z}_{\mathrm{t}}$ & 0.12 & 0.13 & 0.15 & 0.16 & 0.22 & 0.15 & 0.14 & 0.13 \\
\hline $\mathrm{Ba} / \mathrm{Zt}_{\mathrm{t}}$ & 0.44 & 0.33 & 0.34 & 0.26 & 0.31 & 0.34 & 0.35 & 0.25 \\
\hline $\mathrm{CeN}_{\mathrm{N}} / \mathrm{Y}_{\mathrm{N}}$ & 0.74 & 0.96 & 1.23 & 1.14 & 1.61 & 1.23 & 1.09 & 1.00 \\
\hline $\mathrm{Fe}_{\text {tot }} / \mathrm{Mg}$ & 1.64 & 1.81 & 1.25 & 1.50 & 1.72 & 1.48 & 1.79 & 1.42 \\
\hline $\mathrm{K} / \mathrm{Rb}$ & 546 & 371 & 515 & 506 & 445 & 451 & 167 & 971 \\
\hline $\mathrm{Ba} / \mathrm{Sr}$ & 0.20 & 0.15 & 0.16 & 0.11 & 0.14 & 0.16 & 0.17 & 0.12 \\
\hline Q & 0.0 & 0.0 & 0.0 & 0.0 & 0.0 & 0.0 & 0.0 & 0.0 \\
\hline Or & 1.6 & 2.4 & 1.5 & 1.5 & 2.3 & 2.0 & 3.1 & 1.4 \\
\hline$A b$ & 23.2 & 18.6 & 24.1 & 12.1 & 23.9 & 24.3 & 24.4 & 23.4 \\
\hline An & 31.7 & 30.5 & 31.1 & 31.2 & 30.4 & 30.2 & 27.8 & 31.7 \\
\hline $\mathrm{Ne}$ & 0.0 & 2.4 & 0.0 & 4.7 & 0.0 & 0.0 & 0.0 & 0.0 \\
\hline Di & 28.5 & 37.2 & 30.7 & 42.8 & 21.8 & 22.4 & 25.0 & 24.3 \\
\hline Hy & 5.3 & 0.0 & 1.0 & 0.0 & 13.5 & 10.7 & 8.4 & 9.5 \\
\hline Oi & 4.6 & 3.9 & 7.1 & 3.1 & 2.8 & 5.3 & 5.9 & 4.8 \\
\hline Mt & 1.5 & 1.5 & 1.3 & 1.4 & 1.7 & 1.6 & 1.8 & 1.5 \\
\hline II & 2.4 & 2.2 & 2.3 & 2.1 & 2.4 & 2.4 & 2.3 & 2.2 \\
\hline Ap & 0.4 & 0.4 & 0.4 & 0.4 & 0.4 & 0.3 & 0.3 & 0.3 \\
\hline
\end{tabular}

${ }^{a}$ Normative values based on $\mathrm{Fe}_{2} \mathrm{O}_{3} / \mathrm{FeO}$ ratio of $0.15 ; \mathrm{Fe}_{2} \mathrm{O}_{3}$ is total iron as $\mathrm{Fe}_{2} \mathrm{O}_{3}$. $\mathrm{CeN}_{N} / \mathrm{Y}_{\mathrm{N}}$ represents ratio of chondrite-normalized values.

442-1 exhibits a large amount of within-unit variation: $\mathrm{Cr}$ ranges from 193 to over $300 \mathrm{ppm}$ and one lithologic sub-unit (442-1K) contains $412 \mathrm{ppm} \mathrm{Cr}$ and $201 \mathrm{ppm} \mathrm{Ni}$. $\mathrm{Zr}$ varies between 67 and $106 \mathrm{ppm}$, and the $\mathrm{Fe} / \mathrm{Mg}$ ratio varies from 0.97 to 2.41 . The singularly high $\mathrm{Ni}, \mathrm{Cr}$, and normative 01 contents (Figures 3, 5, and 6) and the low $\mathrm{Fe} / \mathrm{Mg}$ ratio and $\mathrm{Zr}$ levels in lithologic sub-unit $442-1 \mathrm{~K}$ (sample 442-8-1, $32 \mathrm{~cm}$ ) may be due to the development of a within-flow cumulus assemblage. The rock at this interval has a doleritic appearance, and there is evidence of a concentration of crystals where the sample was taken.

Geochemical units $442-2$ and $442-4$ have significantly higher $\mathrm{Zr}$ contents and $\mathrm{Fe} / \mathrm{Mg}$ ratios, and complementary lower contents of $\mathrm{Ni}$ and $\mathrm{Cr}$, than 442-1. Geochemical units 442-3 and 442-4 have very low $\mathrm{Cr}$ (35-64 $\mathrm{ppm})$ and $\mathrm{Ni}(32-63 \mathrm{ppm})$ levels, and a high $\mathrm{Fe} / \mathrm{Mg}$ ratio (1.47-2.32), indicative of considerable fractionation of olivine, pyroxene, and (or) chrome spinel prior to eruption and emplacement of the magma. Indeed, none of the geochemical units of Site 442 has the high $\mathrm{MgO}$ $(>9.0 \%)$ and $\mathrm{Ni}(>200 \mathrm{ppm})$ indicative of the "primary" basaltic liquid of Kay et al. (1970), apart 
from 442-8-1, $32 \mathrm{~cm}$, which as we have seen could easily be a cumulus assemblage derived by within-flow differentiation.

The chemical data for Site 442 may be conveniently plotted against an index of fractionation to illustrate variations within and between the four distinct geochemical units. The $\mathrm{Fe} / \mathrm{Mg}$ ratio is one such index that has been used in the past, but the strong dependence of this ratio on the composition of the fractionating phases, rather than their quantity, makes it less desirable as a fractionation index than a suitable incompatible trace element. Incompatible or hygromagmatophile elements are those with bulk-distribution coefficients $(D)$ significantly less than unity; ideally, $D$ should approach zero. Hygromagmatophile (HYG) elements may be grouped according to their relative bulk-distribution coefficients into more-HYG $(D \leq 0.01)$ and less-HYG types $(D \sim$ 0.1 ); in ocean-ridge basalts, the more-HYG elements include $\mathrm{Rb}, \mathrm{K}$, Th, $\mathrm{La}, \mathrm{Ce}, \mathrm{Nb}, \mathrm{Ta}(\mathrm{Zr}, \mathrm{Hf})$; and the lessHYG elements include ( $\mathrm{Zr}, \mathrm{Hf}$ ), Sm, Ti, P, Y, and the heavy rare-earth elements (REE) (Wood et al, 1979). Zirconium is thus considered to have a low $D$ value in ocean-ridge basalts, and probably back-arc basin basalts also (Saunders and Tarney, 1979; Weaver et al., 1979), and in consideration also of its apparent immobility during the hydrous alteration of basalts (Cann, 1970; Pearce and Cann, 1971), it therefore provides a suitable index of fractionation for the study of basaltic suites (cf. Weaver et al., 1972).

Figures 5 to 14 present a visual summary of the data, using zirconium as an index of fractionation. Figure 15 shows down-hole variation in $\mathrm{Zr}, \mathrm{P}_{2} \mathrm{O}_{5}$, and $\mathrm{K}_{2} \mathrm{O}$. Although there is only a moderate range of $\mathrm{Zr}$ contents in the Site 442 basalts, the salient points may be summarized as follows. $\mathrm{TiO}_{2}, \mathrm{Ce}, \mathrm{Nb}$, and $\mathrm{Y}$ (Figure 16) exhibit strong colinearity with $\mathrm{Zr}$, and the ratios do not change significantly from unit to unit. By inference, it may be expected that the other hygromagmatophile elements $\mathrm{P}, \mathrm{K}, \mathrm{Rb}$, and $\mathrm{Ba}$ would also show a colinear relationship with $\mathrm{Zr}$, but in fact this is not the case (Figures 10 to 14). $\mathrm{P}_{2} \mathrm{O}_{5}$ varies independently of $\mathrm{Zr}$ (and of the other incompatible elements) especially in geochemical units 442-2, 442-3, and 442-4 (Figure 10). This is clearly illustrated in Figure 15, where $\mathrm{P}_{2} \mathrm{O}_{5}$ is shown to vary only slightly, and colinearly with $\mathrm{Zr}$, in unit $442-1$, but shows erratic behavior in the other units. We suggest that this variation is due to alteration of the pillow basalts, possibly at very low temperatures $\left(<25^{\circ} \mathrm{C}\right) . \mathrm{K}$, $\mathrm{Rb}, \mathrm{Ba}$, and $\mathrm{Sr}$ show similar erratic behavior, which is probably also due to alteration by sea water: the mobility of these elements in oceanic basalts is well documented (Hart, 1971; Hart et al., 1974).

Included on Table 4 are representative element ratios of basalts being erupted at a present-day ocean ridge, and each biaxial plot in this paper contains ratio lines to aid comparison with Leg 58 basalts. Three distinct types are presented for comparative purposes.

Normal, or $N$-type ocean ridge basalts, which are the so-called mid-ocean-ridge basalts (MORB) now being erupted along most ocean ridges, have low initial ${ }^{87} \mathrm{Sr}$ / ${ }^{86 \mathrm{Sr}}$ ratios (0.7023-0.7027; Hart, 1971), have low abun- dances of many HYG elements $(\mathrm{K}, \mathrm{Rb}, \mathrm{Ba}, \mathrm{Th}, \mathrm{U}$, light REE, Nb and Ta; White and Schilling, 1978; Bougault et al., 1979), and are depleted in the more-HYG elements relative to chondrites (Schilling, 1971; Frey et al., 1974; Wood et al., 1979). Representative analyses of $\mathrm{N}$-type ocean-ridge basalts were taken from Rhodes et al. (1976).

Enriched, or E-type tholeiitic and alkalic basalts, are presently being erupted along certain sections of the mid-Atlantic Ridge (e.g., the Azores, parts of Iceland, and MAR $45^{\circ} \mathrm{N}$ ). They are characterized by variable but generally high abundances of most HYG elements; ratios of the more-HYG elements are approximately chondritic (Wood et al., 1979), and initial ${ }^{87} \mathrm{Sr} / 86 \mathrm{Sr}$ ratios are moderately high (0.7035; White et al., 1976).

Transitional or T-type ridge segments lie between the normal and enriched areas, and include most of Iceland, the Reykjanes Ridge $\left(60-64^{\circ} \mathrm{N}\right)$ and the FAMOUS area (MAR $35-39^{\circ} \mathrm{N}$ ). These segments are erupting basalts with trace-element abundances and isotope chemistry intermediated between $\mathrm{N}$ - and E-type basalts. In addition to ratios for ocean-ridge basalts, representative ratios from basalts from the East Scotia Sea (Saunders and Tarney, 1979) and from the Bransfield Strait (Weaver et al., 1979) back-arc basins are presented in Table 5. The data for the MAR $45^{\circ} \mathrm{N}$ (E-type) and MAR $63^{\circ} \mathrm{N}$ (T-type) basalts are from Tarney et al. (1979) and were determined using the same XRF spectrometer as the present Leg 58 samples, as were the Scotia Sea and Bransfield Strait lavas. Inter-laboratory error is thus avoided.

In $\mathrm{Zr} / \mathrm{Nb}$ ratios (Figure 9), the basalts of Site 442 closely resemble $\mathrm{N}$-type ocean-ridge basalts recovered during DSDP Leg 34 from the Nazca plate (Rhodes et al., 1976). $\mathrm{Ce} / \mathrm{Zr}$ and $\mathrm{TiO}_{2} / \mathrm{Zr}$ ratios are also similar to $\mathrm{N}$-type ocean-ridge basalts, and also closely resemble those from the East Scotia Sea (Figures 7 and 8). Thus, in relative abundances of $\mathrm{Ti}, \mathrm{Zr}, \mathrm{Nb}$, and $\mathrm{Ce}$, the Site 442 basalts most closely resemble $\mathrm{N}$-type ocean-ridge basalts.

Full rare-earth-element data are not yet available for the Site 442 basalts, althoughWood et al. (this volume) have presented some preliminary patterns for Sites 443 , 444 , and 446 . However, by using $\mathrm{Y}$ as a heavy REE, approximately equivalent to $\mathrm{Ho}$, a plot of Ce versus $\mathrm{Y}$ (Figure 16) shows that the Site 442 basalts have chondrite-normalized $\mathrm{Ce} / \mathrm{Y}$ ratios (represented as $\mathrm{Ce}_{\mathrm{N}} / \mathrm{Y}_{\mathrm{N}}$ ) of about 1 (see also Table 2).

Other chemical criteria frequently used to characterize and discriminate between different basalt types are the relative abundances of $\mathrm{K}, \mathrm{Rb}, \mathrm{U}$, Th, and $\mathrm{Ba}$. Unfortunately, apart from $\mathrm{Th}$ and $\mathrm{Ba}$, these elements are easily mobilized during alteration by sea water (Bass et al., 1973; Hart et al., 1974; Mitchell and Aumento, 1977) and from Figures 11, 12, and 13 it is apparent that alteration precludes their use in the present study. However, $\mathrm{Ba}$ levels in the Site 442 basalts are low (generally less than $40 \mathrm{ppm}$ ), giving the low $\mathrm{Ba} / \mathrm{Zr}$ ratios (Figure 13) typical of N-type ocean-ridge basalts. Wood et al. (this volume) have also shown that the $\mathrm{Th} / \mathrm{Hf}$ ratios of the Site 442 basalts are similar to those of $\mathrm{N}$-type basalts. 
TABLE 3

Analyses, Element Ratios, and CIPW Norms for Basalts from Hole $442 \mathrm{~B}^{\mathrm{a}}$

\begin{tabular}{|c|c|c|c|c|c|c|c|c|c|c|c|c|}
\hline $\begin{array}{l}\text { Core } \\
\text { Section } \\
\text { Interval (cm) }\end{array}$ & $\begin{array}{r}3 \\
3 \\
40\end{array}$ & $\begin{array}{r}3 \\
3 \\
140\end{array}$ & $\begin{array}{r}3 \\
4 \\
13\end{array}$ & $\begin{array}{r}4 \\
1 \\
57\end{array}$ & $\begin{array}{r}4 \\
2 \\
110\end{array}$ & $\begin{array}{r}4 \\
3 \\
21\end{array}$ & $\begin{array}{r}5 \\
1 \\
120\end{array}$ & $\begin{array}{l}5 \\
2 \\
1\end{array}$ & $\begin{array}{r}5 \\
2 \\
60\end{array}$ & $\begin{array}{r}5 \\
2 \\
106\end{array}$ & $\begin{array}{r}5 \\
3 \\
94\end{array}$ & $\begin{array}{r}5 \\
4 \\
79\end{array}$ \\
\hline $\mathrm{SiO}_{2}(\%)$ & 50.2 & 50.0 & 50.0 & 49.5 & 50.2 & 49.5 & 50.0 & 49.9 & 50.2 & 49.8 & 49.6 & 49.6 \\
\hline $\mathrm{TiO}_{2}$ & 1.38 & 1.23 & 1.27 & 1.18 & 1.19 & 1.18 & 1.29 & 1.23 & 1.26 & 1.15 & 1.18 & 1.18 \\
\hline $\mathrm{Al}_{2} \mathrm{O}_{3}$ & 15.9 & 16.5 & 15.9 & 16.5 & 16.0 & 15.9 & 16.0 & 15.9 & 15.9 & 15.8 & 15.6 & 16.0 \\
\hline $\mathrm{Fe}_{2} \mathrm{O}_{3}$ & 9.23 & 9.07 & 9.98 & 8.87 & 8.91 & 9.17 & 8.74 & 8.48 & 8.66 & 8.39 & 8.59 & 9.01 \\
\hline $\mathrm{MnO}$ & 0.17 & 0.13 & 0.14 & 0.15 & 0.11 & 0.12 & 0.11 & 0.10 & 0.10 & 0.11 & 0.12 & 0.12 \\
\hline $\mathrm{MgO}$ & 6.08 & 6.63 & 6.39 & 6.00 & 6.46 & 6.64 & 7.49 & 7.63 & 7.93 & 7.32 & 6.71 & 6.54 \\
\hline $\mathrm{CaO}$ & 12.15 & 12.49 & 11.69 & 12.82 & 11.98 & 12.42 & 11.60 & 11.43 & 11.11 & 12.30 & 12.84 & 12.80 \\
\hline $\mathrm{Na}_{2} \mathrm{O}$ & 2.72 & 2.76 & 2.91 & 2.88 & 3.03 & 3.27 & 2.89 & 2.97 & 2.80 & 2.79 & 2.78 & 2.70 \\
\hline $\mathrm{K}_{2} \mathrm{O}$ & 0.36 & 0.29 & 0.33 & 0.31 & 0.33 & 0.29 & 0.25 & 0.25 & 0.22 & 0.34 & 0.31 & 0.23 \\
\hline $\mathrm{P}_{2} \mathrm{O}_{5}$ & 0.18 & 0.15 & 0.15 & 0.17 & 0.14 & 0.14 & 0.14 & 0.14 & 0.13 & 0.13 & 0.14 & 0.14 \\
\hline Total & 98.44 & 99.19 & 98.83 & 98.40 & 98.33 & 98.58 & 98.54 & 98.00 & 98.37 & 98.16 & 97.86 & 98.37 \\
\hline $\mathrm{Ni}(\mathrm{ppm})$ & 44 & 57 & 52 & 60 & 42 & 53 & 84 & 103 & 157 & 80 & 74 & 60 \\
\hline $\mathrm{Cr}$ & 193 & 247 & 221 & 258 & 222 & 216 & 222 & 237 & 232 & 244 & 226 & 233 \\
\hline $\mathrm{Zn}$ & 76 & 83 & 85 & 70 & 66 & 67 & 67 & 70 & 84 & 59 & 100 & 79 \\
\hline $\mathrm{Ga}$ & 20 & 18 & 18 & 18 & 20 & 17 & 18 & 19 & 19 & 15 & 15 & 14 \\
\hline $\mathrm{Rb}$ & 8 & 5 & 7 & 7 & 8 & 6 & 3 & 2 & 2 & 5 & 5 & 3 \\
\hline $\mathrm{Sr}$ & 192 & 186 & 180 & 189 & 187 & 184 & 188 & 183 & 179 & 176 & 179 & 185 \\
\hline $\mathrm{Y}$ & 32 & 29 & 30 & 30 & 29 & 28 & 25 & 23 & 27 & 26 & 27 & 28 \\
\hline $\mathrm{Zr}$ & 98 & 89 & 96 & 89 & 85 & 90 & 89 & 91 & 91 & 84 & 90 & 84 \\
\hline $\mathrm{Nb}$ & $<1$ & 1 & 1 & 4 & 3 & 2 & $<1$ & 1 & 4 & 3 & 3 & 2 \\
\hline $\mathrm{Ba}$ & 40 & 24 & 37 & 22 & 28 & 26 & 33 & 32 & 35 & 30 & 32 & 29 \\
\hline $\mathrm{La}$ & 4 & 2 & 5 & 3 & 3 & 2 & 5 & 2 & 2 & 2 & 3 & 3 \\
\hline $\mathrm{Ce}$ & 14 & 16 & 12 & 17 & 12 & 14 & 13 & 12 & 11 & 11 & 9 & 16 \\
\hline $\mathrm{Pb}$ & 3 & 7 & 1 & 6 & 3 & 4 & $<1$ & $<1$ & 1 & 1 & 4 & 3 \\
\hline Th & 2 & $<1$ & 1 & 2 & $<1$ & $<1$ & 2 & $<1$ & $<1$ & $<1$ & 1 & 2 \\
\hline $\mathrm{Zr} / \mathrm{Nb}$ & - & 89 & 96 & 22 & 28 & 45 & - & 91 & 23 & 28 & 30 & 42 \\
\hline $\mathrm{Ti} / \mathrm{Zr}$ & 84 & 83 & 79 & 79 & 84 & 79 & 87 & 81 & 83 & 82 & 79 & 84 \\
\hline $\mathrm{Y} / \mathrm{Zr}$ & 0.33 & 0.33 & 0.31 & 0.34 & 0.34 & 0.31 & 0.28 & 0.25 & 0.30 & 0.31 & 0.30 & 0.33 \\
\hline $\mathrm{Ce} / \mathrm{Zr}$ & 0.14 & 0.18 & 0.13 & 0.19 & 0.14 & 0.16 & 0.15 & 0.13 & 0.12 & 0.13 & 0.10 & 0.19 \\
\hline $\mathrm{Ba} / \mathrm{Zr}$ & 0.41 & 0.27 & 0.39 & 0.25 & 0.33 & 0.29 & 0.37 & 0.35 & 0.38 & 0.36 & 0.36 & 0.35 \\
\hline $\mathrm{Ce}_{\mathrm{N}} / \mathrm{Y}_{\mathrm{N}}$ & 1.07 & 1.36 & 0.98 & 1.39 & 1.02 & 1.23 & 1.28 & 1.28 & 1.00 & 1.04 & 0.82 & 1.40 \\
\hline $\mathrm{Fe}_{\text {tot }} / \mathrm{Mg}$ & 1.76 & 1.59 & 1.81 & 1.71 & 1.60 & 1.60 & 1.35 & 1.29 & 1.27 & 1.33 & 1.48 & 1.60 \\
\hline $\mathrm{K} / \mathrm{Rb}$ & 370 & 476 & 394 & 371 & 344 & 405 & 695 & 1021 & 897 & 566 & 523 & 636 \\
\hline $\mathrm{Ba} / \mathrm{Sr}$ & 0.21 & 0.13 & 0.21 & 0.12 & 0.15 & 0.14 & 0.18 & 0.17 & 0.20 & 0.17 & 0.18 & 0.16 \\
\hline Q & 0.0 & 0.0 & 0.0 & 0.0 & 0.0 & 0.0 & 0.0 & 0.0 & 0.0 & 0.0 & 0.0 & 0.0 \\
\hline Or & 2.1 & 1.7 & 2.0 & 1.9 & 2.0 & 1.8 & 1.5 & 1.5 & 1.3 & 2.1 & 1.9 & 1.4 \\
\hline $\mathrm{Ab}$ & 23.4 & 23.5 & 24.9 & 24.8 & 26.1 & 26.5 & 24.8 & 25.6 & 24.1 & 24.0 & 24.0 & 23.2 \\
\hline An & 30.7 & 32.0 & 29.8 & 31.6 & 29.6 & 28.2 & 30.5 & 30.0 & 30.7 & 30.2 & 29.7 & 31.4 \\
\hline $\mathrm{Ne}$ & 0.0 & 0.0 & 0.0 & 0.0 & 0.0 & 0.9 & 0.0 & 0.0 & 0.0 & 0.0 & 0.0 & 0.0 \\
\hline $\mathrm{Di}$ & 24.1 & 24.1 & 22.9 & 26.1 & 24.5 & 27.3 & 21.9 & 21.9 & 19.8 & 25.3 & 28.0 & 26.3 \\
\hline $\mathrm{Hy}$ & 13.9 & 8.4 & 10.4 & 4.4 & 7.5 & 0.0 & 10.2 & 9.3 & 15.2 & 7.0 & 5.5 & 8.1 \\
\hline $\mathrm{Ol}$ & 0.2 & 5.1 & 4.6 & 6.2 & 5.3 & 10.4 & 5.9 & 6.8 & 3.8 & 6.7 & 5.8 & 4.5 \\
\hline Mt & 1.6 & 1.6 & 1.8 & 1.6 & 1.6 & 1.6 & 1.5 & 1.5 & 1.5 & 1.5 & 1.5 & 1.6 \\
\hline Il & 2.7 & 2.4 & 2.4 & 2.3 & 2.3 & 2.3 & 2.5 & 2.4 & 2.4 & 2.2 & 2.3 & 2.3 \\
\hline Ap & 0.4 & 0.4 & 0.4 & 0.4 & 0.3 & 0.3 & 0.3 & 0.3 & 0.3 & 0.3 & 0.3 & 0.3 \\
\hline
\end{tabular}

The colinearity exhibited by the hygromagmatophile elements (excluding those which have been subject to mobilization during alteration by sea water) in the Site 442 basalts suggests that the magmas giving rise to the four geochemical units were derived from similar parental magmas, or by fractional crystallization of a common parental magma. However, unit 442-1 could not have been derived from the same magma that produced the other units, because it is separated from them by 2 meters of mudstone. This could have taken as long as 2 m.y. to have been deposited (Site 442 report, this volume). In addition, units 442-3 and 442-2 are separated by a magnetic reversal, which suggests that these two units may not be comagmatic, although they are probably cogenetic.

\section{Site 443}

Site 443 (Figure 1) lies approximately $95 \mathrm{~km}$ east of the axial zone of the Shikoku Basin, at $29^{\circ} 19.65^{\prime} \mathrm{N}$, $137^{\circ} 26.43^{\prime} \mathrm{E}$, at a water depth of 4372 meters. Approximately 116 meters of basaltic flows and 460 meters of sediment were drilled in the single hole. The site is on a positive magnetic lineation tentatively identified as anomaly 6A (21-22 m.y.; LaBrecque et al., 1977; Kobayashi and Nakata, 1977). The oldest microfossils recovered at this site indicate an age of 14 to 17 m.y., but it is 
TABLE 3 - Continued

\begin{tabular}{|c|c|c|c|c|c|c|c|c|c|c|c|c|c|}
\hline $\begin{array}{r}6 \\
1 \\
53\end{array}$ & $\begin{array}{r}6 \\
2 \\
32\end{array}$ & $\begin{array}{r}6 \\
2 \\
147\end{array}$ & $\begin{array}{r}6 \\
3 \\
28\end{array}$ & $\begin{array}{r}7 \\
1 \\
46\end{array}$ & $\begin{array}{r}7 \\
1 \\
110\end{array}$ & $\begin{array}{r}8 \\
1 \\
32\end{array}$ & $\begin{array}{r}8 \\
5 \\
74\end{array}$ & $\begin{array}{r}8 \\
6 \\
141\end{array}$ & $\begin{array}{r}8 \\
7 \\
104\end{array}$ & $\begin{array}{r}9 \\
1 \\
34\end{array}$ & $\begin{array}{r}9 \\
1 \\
107\end{array}$ & $\begin{array}{r}9 \\
2 \\
37\end{array}$ & $\begin{array}{r}9 \\
3 \\
54\end{array}$ \\
\hline 49.7 & 49.8 & 49.8 & 50.1 & 50.3 & 50.2 & 47.6 & 49.4 & 49.5 & 50.2 & 49.3 & 49.1 & 49.2 & 50.2 \\
\hline 1.19 & 1.16 & 1.19 & 1.24 & 1.20 & 1.35 & 0.91 & 1.16 & 1.24 & 1.21 & 1.14 & 1.08 & 1.13 & 1.47 \\
\hline 15.6 & 15.6 & 15.8 & 16.3 & 15.7 & 15.8 & 14.9 & 15.5 & 15.2 & 16.0 & 16.1 & 14.8 & 15.3 & 15.0 \\
\hline 8.98 & 9.05 & 8.49 & 8.03 & 9.92 & 10.05 & 9.88 & 9.51 & 9.38 & 8.90 & 8.31 & 9.17 & 8.42 & 11.08 \\
\hline 0.12 & 0.13 & 0.11 & 0.13 & 0.16 & 0.16 & 0.18 & 0.15 & 0.13 & 0.13 & 0.14 & 0.13 & 0.12 & 0.14 \\
\hline 6.89 & 6.97 & 8.19 & 7.14 & 7.94 & 7.58 & 11.83 & 8.88 & 9.28 & 7.43 & 7.78 & 7.64 & 6.73 & 7.85 \\
\hline 12.94 & 12.88 & 10.97 & 11.90 & 10.19 & 10.51 & 9.38 & 10.34 & 10.13 & 10.73 & 10.97 & 12.50 & 13.98 & 10.03 \\
\hline 2.78 & 2.73 & 2.72 & 2.99 & 2.82 & 2.78 & 2.09 & 2.64 & 2.70 & 3.24 & 2.59 & 2.66 & 2.72 & 2.97 \\
\hline 0.25 & 0.26 & 0.20 & 0.29 & 0.20 & 0.17 & 0.11 & 0.11 & 0.15 & 0.37 & 0.26 & 0.52 & 0.33 & 0.30 \\
\hline 0.15 & 0.15 & 0.13 & 0.15 & 0.16 & 0.14 & 0.12 & 0.13 & 0.16 & 0.14 & 0.12 & 0.13 & 0.14 & 0.17 \\
\hline 98.63 & 98.73 & 97.64 & 98.20 & 98.61 & 98.68 & 96.96 & 97.86 & 97.84 & 98.38 & 96.74 & 97.80 & 98.07 & 99.21 \\
\hline 64 & 71 & 82 & 49 & 50 & 59 & 201 & 100 & 105 & 66 & 137 & 118 & 100 & 118 \\
\hline 237 & 277 & 247 & 232 & 209 & 211 & 412 & 255 & 258 & 228 & 300 & 282 & 270 & 251 \\
\hline 75 & 106 & 71 & 76 & 75 & 80 & 59 & 58 & 63 & 41 & 59 & 61 & 85 & 99 \\
\hline 18 & 18 & 20 & 18 & 19 & 18 & 19 & 16 & 18 & 17 & 18 & 14 & 17 & 20 \\
\hline 2 & 7 & $<1$ & 3 & 2 & 2 & $<1$ & 2 & $<1$ & 5 & 11 & 9 & 7 & 3 \\
\hline 130 & 152 & 193 & 195 & 202 & 161 & 135 & 159 & 169 & 154 & 167 & 164 & 173 & 177 \\
\hline 28 & 27 & 28 & 26 & 31 & 30 & 21 & 28 & 27 & 20 & 24 & 25 & 28 & 31 \\
\hline 88 & 86 & 83 & 89 & 94 & 93 & 67 & 84 & 91 & 94 & 78 & 79 & 84 & 106 \\
\hline 3 & 2 & 4 & 3 & 3 & 4 & 6 & 1 & 1 & 4 & 4 & 6 & 4 & 2 \\
\hline 29 & 37 & 28 & 32 & 44 & 40 & 14 & 29 & 34 & 19 & 35 & 35 & 52 & 31 \\
\hline 3 & 3 & 4 & 4 & 4 & 3 & 5 & 3 & 3 & 2 & 5 & 2 & 2 & 3 \\
\hline 10 & 8 & 12 & 12 & 14 & 10 & 13 & 13 & 12 & 13 & 14 & 10 & 12 & 20 \\
\hline 3 & 8 & 1 & 6 & 3 & 5 & 1 & 4 & 5 & 6 & 5 & 4 & 6 & 3 \\
\hline$<1$ & $<1$ & $<1$ & 1 & $<1$ & $<1$ & $<1$ & $<1$ & 2 & $<1$ & 1 & $<1$ & 2 & 1 \\
\hline 29 & 43 & 21 & 30 & 31 & 23 & 11 & 84 & 91 & 23 & 19 & 13 & 21 & 53 \\
\hline 81 & 81 & 86 & 84 & 77 & 87 & 82 & 83 & 82 & 77 & 88 & 82 & 81 & 83 \\
\hline 0.32 & 0.31 & 0.34 & 0.29 & 0.33 & 0.32 & 0.31 & 0.33 & 0.30 & 0.21 & 0.31 & 0.32 & 0.33 & 0.29 \\
\hline 0.11 & 0.09 & 0.14 & 0.13 & 0.15 & 0.11 & 0.19 & 0.15 & 0.13 & 0.14 & 0.18 & 0.13 & 0.14 & 0.19 \\
\hline 0.33 & 0.43 & 0.34 & 0.36 & 0.47 & 0.43 & 0.21 & 0.35 & 0.37 & 0.20 & 0.45 & 0.44 & 0.62 & 0.29 \\
\hline 0.88 & 0.73 & 1.05 & 1.13 & 1.11 & 0.82 & 1.52 & 1.14 & 1.09 & 1.60 & 1.43 & 0.98 & 1.05 & 1.58 \\
\hline 1.51 & 1.51 & 1.20 & 1.30 & 1.45 & 1.54 & 0.97 & 1.24 & 1.17 & 1.39 & 1.24 & 1.39 & 1.45 & 1.64 \\
\hline 1042 & 311 & - & 805 & 822 & 693 & - & 469 & - & 618 & 192 & 478 & 396 & 830 \\
\hline 0.22 & 0.24 & 0.15 & 0.16 & 0.22 & 0.25 & 0.10 & 0.18 & 0.20 & 0.12 & 0.21 & 0.21 & 0.30 & 0.18 \\
\hline 0.0 & 0.0 & 0.0 & 0.0 & 0.0 & 0.0 & 0.0 & 0.0 & 0.0 & 0.0 & 0.0 & 0.0 & 0.0 & 0.0 \\
\hline 1.5 & 1.6 & 1.2 & 1.8 & 1.2 & 1.0 & 0.6 & 0.7 & 0.9 & 2.2 & 1.6 & 3.1 & 2.0 & 1.8 \\
\hline 23.8 & 23.4 & 23.6 & 25.8 & 24.2 & 23.8 & 18.2 & 22.8 & 23.3 & 27.9 & 22.7 & 23.0 & 22.5 & 25.3 \\
\hline 29.7 & 29.8 & 31.1 & 30.7 & 30.2 & 30.4 & 31.8 & 30.9 & 29.6 & 28.6 & 32.7 & 27.6 & 29.2 & 26.9 \\
\hline 0.0 & 0.0 & 0.0 & 0.0 & 0.0 & 0.0 & 0.0 & 0.0 & 0.0 & 0.0 & 0.0 & 0.0 & 0.5 & 0.0 \\
\hline 28.0 & 27.7 & 19.3 & 23.1 & 16.4 & 17.6 & 12.4 & 16.8 & 16.8 & 20.0 & 18.4 & 28.5 & 33.1 & 18.1 \\
\hline 5.5 & 6.6 & 15.8 & 7.7 & 19.6 & 19.4 & 19.6 & 17.9 & 17.5 & 7.6 & 16.8 & 2.9 & 0.0 & 16.2 \\
\hline 6.4 & 6.0 & 4.2 & 6.1 & 3.2 & 2.1 & 12.6 & 5.8 & 6.6 & 8.7 & 3.1 & 10.0 & 8.0 & 5.5 \\
\hline 1.6 & 1.6 & 1.5 & 1.4 & 1.8 & 1.8 & 1.8 & 1.7 & 1.7 & 1.6 & 1.5 & 1.6 & 1.5 & 1.9 \\
\hline 2.3 & 2.2 & 2.3 & 2.4 & 2.3 & 2.6 & 1.8 & 2.3 & 2.4 & 2.3 & 2.2 & 2.1 & 2.2 & 2.8 \\
\hline 0.4 & 0.4 & 0.3 & 0.3 & 0.4 & 0.3 & 0.3 & 0.3 & 0.4 & 0.3 & 0.3 & 0.3 & 0.3 & 0.4 \\
\hline
\end{tabular}

possible that some older sediments were not cored (Site 443 report, this volume). Sites 442 and 443 lie on the same spreading line, on opposite sides of the spreading axis of the basins.

The basalts recovered at Site 443 appear to be from massive flows or sills (Figure 17). Essentially all the basalts are olivine-bearing, and in general vesicles are scarce (although in lithologic unit 3 they reach $30 \%$ ). On the basis of shipboard petrography, the basalt sequence has been divided into six lithologic units. Unit 1 consists of one small piece of glassy basalt immediately underlying the sediments in Core 49 . Unit 2 comprises eight sub-units, each of which is considered to be an in- dividual, massive basalt flow. The various sub-units have similar mineralogies and textures, containing both olivine and plagioclase phenocrysts, having less than 5 per cent vesicles, and ranging in texture from cryptocrystalline at chilled margins to intergranular and even subophitic at the centers of the thickest flows. The most massive sub-unit, $2 \mathrm{E}$, shows accumulation of olivine in its middle and lower sections, indicative of gravitational differentiation.

Unit 3 has been divided into nine sub-units, the six uppermost comprising three pillow-basalt sub-units $(3 \mathrm{~A}, 3 \mathrm{C}, 3 \mathrm{E})$ each with an underlying massive-basalt sub-unit $(3 \mathrm{~B}, 3 \mathrm{D}, 3 \mathrm{~F})$ of similar lithology. The pillow 
TABLE 3 - Continued

\begin{tabular}{|c|c|c|c|c|c|c|c|c|c|c|c|c|}
\hline $\begin{array}{l}\text { Core } \\
\text { Section } \\
\text { Interval }(\mathrm{cm})\end{array}$ & $\begin{array}{r}10 \\
1 \\
110\end{array}$ & $\begin{array}{r}11 \\
1 \\
146\end{array}$ & $\begin{array}{r}11 \\
2 \\
70\end{array}$ & $\begin{array}{r}11 \\
3 \\
24\end{array}$ & $\begin{array}{r}12 \\
1 \\
52\end{array}$ & $\begin{array}{r}13 \\
1 \\
34\end{array}$ & $\begin{array}{r}13 \\
2 \\
92\end{array}$ & $\begin{array}{r}13 \\
3 \\
19\end{array}$ & $\begin{array}{r}15 \\
1 \\
40\end{array}$ & $\begin{array}{r}16 \\
1 \\
24\end{array}$ & $\begin{array}{r}16 \\
1 \\
47\end{array}$ & $\begin{array}{r}16 \\
1 \\
111\end{array}$ \\
\hline $\mathrm{SiO}_{2}(\%)$ & 49.9 & 48.8 & 49.8 & 49.8 & 49.1 & 49.9 & 49.2 & 49.5 & 49.7 & 50.0 & 49.7 & 50.2 \\
\hline $\mathrm{TiO}_{2}$ & 1.62 & 1.60 & 1.55 & 1.59 & 1.60 & 1.74 & 1.66 & 1.58 & 1.23 & 1.31 & 1.23 & 1.13 \\
\hline $\mathrm{Al}_{2} \mathrm{O}_{3}$ & 16.0 & 16.4 & 16.1 & 16.3 & 16.3 & 16.1 & 16.1 & 16.1 & 16.3 & 16.7 & 16.6 & 16.3 \\
\hline $\mathrm{Fe}_{2} \mathrm{O}_{3}$ & 10.94 & 11.89 & 10.34 & 10.31 & 11.48 & 10.94 & 11.45 & 10.79 & 9.88 & 10.67 & 9.83 & 8.61 \\
\hline $\mathrm{MnO}$ & 0.17 & 0.19 & 0.16 & 0.18 & 0.19 & 0.19 & 0.16 & 0.14 & 0.16 & 0.16 & 0.17 & 0.12 \\
\hline $\mathrm{MgO}$ & 5.26 & 4.52 & 5.89 & 5.56 & 4.66 & 5.60 & 4.66 & 5.20 & 5.52 & 5.58 & 5.51 & 6.80 \\
\hline $\mathrm{CaO}$ & 12.18 & 12.43 & 11.97 & 12.17 & 12.12 & 12.01 & 12.45 & 12.27 & 12.65 & 11.70 & 12.67 & 12.17 \\
\hline $\mathrm{Na}_{2} \mathrm{O}$ & 2.89 & 2.66 & 2.83 & 2.86 & 2.59 & 2.75 & 2.73 & 2.79 & 2.45 & 2.83 & 2.39 & 2.58 \\
\hline $\mathrm{K}_{2} \mathrm{O}$ & 0.34 & 0.41 & 0.33 & 0.33 & 0.50 & 0.36 & 0.39 & 0.40 & 0.39 & 0.48 & 0.38 & 0.30 \\
\hline $\mathrm{P}_{2} \mathrm{O}_{5}$ & 0.21 & 0.29 & 0.22 & 0.23 & 0.32 & 0.25 & 0.33 & 0.35 & 0.40 & 0.19 & 0.22 & 0.14 \\
\hline Total & 99.52 & 99.18 & 99.18 & 99.31 & 98.85 & 99.82 & 99.16 & 99.11 & 98.73 & 99.55 & 98.67 & 98.31 \\
\hline $\mathrm{Ni}(\mathrm{ppm})$ & 50 & 58 & 70 & 71 & 67 & 66 & 48 & 51 & 32 & 39 & 42 & 59 \\
\hline $\mathrm{Cr}$ & 150 & 192 & 195 & 207 & 194 & 201 & 197 & 195 & 49 & 35 & 52 & 50 \\
\hline $\mathrm{Zn}$ & 94 & 108 & 105 & 110 & 99 & 100 & 103 & 110 & 83 & 88 & 79 & 82 \\
\hline $\mathrm{Ga}$ & 20 & 23 & 21 & 21 & 24 & 20 & 21 & 22 & 21 & 22 & 19 & 20 \\
\hline $\mathrm{Rb}$ & 7 & 9 & 5 & 5 & 8 & 6 & 6 & 7 & 7 & 21 & 8 & 4 \\
\hline $\mathrm{Sr}$ & 162 & 185 & 175 & 178 & 181 & 171 & 186 & 182 & 219 & 208 & 210 & 200 \\
\hline Y & 35 & 38 & 37 & 39 & 40 & 43 & 41 & 39 & 31 & 33 & 33 & 27 \\
\hline $\mathrm{Zr}$ & 105 & 114 & 116 & 115 & 120 & 124 & 114 & 115 & 88 & 95 & 88 & 81 \\
\hline $\mathrm{Nb}$ & 3 & 3 & 4 & 5 & 3 & 5 & 4 & 4 & 3 & 2 & 2 & 3 \\
\hline $\mathrm{Ba}$ & 25 & 30 & 24 & 39 & 36 & 28 & 40 & 31 & 33 & 45 & 31 & 30 \\
\hline $\mathrm{La}$ & 6 & 6 & 4 & 4 & 4 & 6 & 6 & 8 & 7 & 5 & 4 & 4 \\
\hline $\mathrm{Ce}$ & 13 & 15 & 18 & 14 & 17 & 19 & 18 & 18 & 15 & 17 & 13 & 16 \\
\hline $\mathrm{Pb}$ & 5 & 3 & 7 & 4 & 2 & 6 & 3 & 2 & 3 & 3 & 4 & 5 \\
\hline Th & 1 & $<1$ & 1 & $<1$ & $<1$ & $<1$ & 2 & 1 & $<1$ & 2 & $<1$ & 4 \\
\hline $\mathrm{Zr} / \mathrm{Nb}$ & 35 & 38 & 29 & 23 & 40 & 25 & 28 & 29 & 29 & 48 & 44 & 27 \\
\hline $\mathrm{Ti} / \mathrm{Zr}$ & 92 & 84 & 80 & 83 & 80 & 84 & 87 & 82 & 84 & 83 & 84 & 84 \\
\hline $\mathrm{Y} / \mathrm{Zr}$ & 0.33 & 0.33 & 0.32 & 0.34 & 0.33 & 0.35 & 0.36 & 0.34 & 0.35 & 0.35 & 0.38 & 0.33 \\
\hline $\mathrm{Ce} / \mathrm{Zr}$ & 0.12 & 0.13 & 0.16 & 0.12 & 0.14 & 0.15 & 0.16 & 0.16 & 0.17 & 0.18 & 0.15 & 0.20 \\
\hline $\mathrm{Ba} / \mathrm{Zr}$ & 0.24 & 0.26 & 0.21 & 0.34 & 0.30 & 0.23 & 0.35 & 0.27 & 0.38 & 0.47 & 0.35 & 0.37 \\
\hline $\mathrm{Ce}_{\mathrm{N}} / \mathrm{Y}_{\mathrm{N}}$ & 0.91 & 0.97 & 1.19 & 0.88 & 1.04 & 1.09 & 1.08 & 1.13 & 1.19 & 1.27 & 0.97 & 1.46 \\
\hline $\mathrm{Fe}_{\text {tot }} / \mathrm{Mg}$ & 2.41 & 3.05 & 2.04 & 2.15 & 2.86 & 2.27 & 2.85 & 2.41 & 2.08 & 2.22 & 2.07 & 1.47 \\
\hline $\mathrm{K} / \mathrm{Rb}$ & 404 & 379 & 553 & 541 & 521 & 495 & 540 & 473 & 464 & 188 & 396 & 623 \\
\hline $\mathrm{Ba} / \mathrm{Sr}$ & 0.15 & 0.16 & 0.14 & 0.22 & 0.20 & 0.16 & 0.22 & 0.17 & 0.15 & 0.22 & 0.15 & 0.15 \\
\hline Q & 0.0 & 0.0 & 0.0 & 0.0 & 0.0 & 0.0 & 0.0 & 0. & 0. & 0.0 & 0.3 & 0.0 \\
\hline Or & 2.0 & 2.4 & 2.0 & 1.9 & 3.0 & 2.1 & 2.3 & 2.4 & 2.3 & 2.8 & 2.3 & 1.8 \\
\hline $\mathrm{Ab}$ & 24.6 & 22.7 & 24.1 & 24.4 & 22.2 & 23.3 & 23.3 & 23.8 & 21.0 & 24.1 & 20.5 & 22.2 \\
\hline An & 29.8 & 31.9 & 30.5 & 30.9 & 31.6 & 30.6 & 30.8 & 30.6 & 32.8 & 31.5 & 33.9 & 32.5 \\
\hline $\mathrm{Ne}$ & 0.0 & 0.0 & 0.0 & 0.0 & 0.0 & 0.0 & 0.0 & 0.0 & 0.0 & 0.0 & 0.0 & 0.0 \\
\hline Di & 24.4 & 23.7 & 22.9 & 23.4 & 22.5 & 22.7 & 24.4 & 23.6 & 23.2 & 21.1 & 23.4 & 22.8 \\
\hline $\mathrm{Hy}$ & 10.2 & 9.5 & 11.1 & 10.1 & 13.4 & 13.0 & 10.8 & 10.6 & 14.3 & 10.7 & 14.1 & 14.7 \\
\hline OI & 2.5 & 2.9 & 3.1 & 3.0 & 0.4 & 1.5 & 1.4 & 2.3 & 0.0 & 4.0 & 0.0 & 1.2 \\
\hline Mt & 1.9 & 2.1 & 1.8 & 1.8 & 2.0 & 1.9 & 2.0 & 1.9 & 1.7 & 1.9 & 1.7 & 1.5 \\
\hline Il & 3.1 & 3.1 & 3.0 & 3.0 & 3.1 & 3.3 & 3.2 & 3.0 & 2.4 & 2.5 & 2.4 & 2.2 \\
\hline $\mathrm{Ap}$ & 0.5 & 0.7 & 0.5 & 0.5 & 0.8 & 0.6 & 0.8 & 0.8 & 0.9 & 0.5 & 0.5 & 0.3 \\
\hline
\end{tabular}

basalts are sparsely olivine- and plagioclase-phyric, and chrome spinel occurs as an accessory phase. Vesicles are common to abundant (15-30\%). The flows are several meters thick, and the thickest again shows subophitic textures and olivine accumulation in its middle and lower sections. The bottom three sub-units of unit 3 are sparsely phyric massive flows or sills, in lithology similar to sub-units $3 \mathrm{~A}$ to $3 \mathrm{~F}$, although they do contain 2 to 3 per cent resorbed plagioclase phenocrysts.

Unit 4 is a single 15 -m-thick flow or sill consisting of intersertal to subophitic basalt. Plagioclase and olivine phenocrysts are common, and pilotaxitic alignment of groundmass pyroxene and feldspar is observed. Unit 5, on the other hand, consists of five flows which are either aphyric or sparsely plagioclase-phyric. Again, however, vesicles are scarce. The last unit drilled, unit 6, comprises two olivine- and plagioclase-phyric basalt subunits, both of which could be flows or sills. Only the top few centimeters of subunit $6 \mathrm{~B}$ were recovered.

The Site 443 basalts are generally fine to medium grained with intersertal to intergranular textures. Porphyritic varieties contain 1 to 10 per cent plagioclase phenocrysts $\left(\mathrm{An}_{70}\right.$ or greater), and 2 to 5 per cent olivine phenocrysts (probably $\mathrm{Fo}_{80-90}$ ). Due to alteration, the presence of groundmass olivine is uncertain. Plagioclase $\left(\mathrm{An}_{60-70}\right)$ and augite are the two major groundmass phases, with about 2 to 5 per cent interstitial magnetite. Finer-grained sections have a groundmass containing 
TABLE 3 - Continued

\begin{tabular}{|c|c|c|c|c|c|c|c|c|}
\hline $\begin{array}{r}16 \\
2 \\
15\end{array}$ & $\begin{array}{r}17 \\
1 \\
14\end{array}$ & $\begin{array}{r}17 \\
1 \\
64\end{array}$ & $\begin{array}{r}17 \\
1 \\
142\end{array}$ & $\begin{array}{r}18 \\
1 \\
46\end{array}$ & $\begin{array}{r}19 \\
1 \\
45\end{array}$ & $\begin{array}{r}19 \\
2 \\
36\end{array}$ & $\begin{array}{r}19 \\
2 \\
51\end{array}$ & $\begin{array}{r}20 \\
1 \\
50\end{array}$ \\
\hline 49.4 & 49.6 & 50.1 & 50.2 & 50.2 & 50.7 & 50.4 & 50.2 & 50.1 \\
\hline 1.26 & 1.24 & 1.21 & 1.65 & 1.63 & 1.65 & 1.65 & 1.59 & 1.62 \\
\hline 15.8 & 16.5 & 17.1 & 15.5 & 16.0 & 15.8 & 16.4 & 16.2 & 15.9 \\
\hline 9.39 & 10.15 & 9.49 & 11.09 & 10.21 & 10.33 & 10.11 & 10.38 & 10.96 \\
\hline 0.13 & 0.15 & 0.15 & 0.17 & 0.16 & 0.15 & 0.16 & 0.19 & 0.17 \\
\hline 6.26 & 5.54 & 5.38 & 5.75 & 5.53 & 6.05 & 5.38 & 5.46 & 5.47 \\
\hline 11.31 & 12.66 & 12.95 & 11.95 & 12.31 & 11.41 & 12.26 & 12.13 & 12.04 \\
\hline 2.61 & 2.37 & 2.70 & 2.68 & 2.82 & 3.24 & 3.08 & 3.13 & 2.76 \\
\hline 0.42 & 0.37 & 0.48 & 0.33 & 0.29 & 0.32 & 0.32 & 0.38 & 0.47 \\
\hline 0.15 & 0.21 & 0.19 & 0.25 & 0.26 & 0.18 & 0.21 & 0.20 & 0.22 \\
\hline 96.71 & 98.79 & 99.78 & 99.61 & 99.42 & 99.81 & 99.92 & 99.86 & 99.70 \\
\hline 39 & 43 & 50 & 47 & 48 & 59 & 57 & 63 & 41 \\
\hline 42 & 57 & 58 & 59 & 59 & 58 & 59 & 60 & 64 \\
\hline 109 & 78 & 149 & 94 & 88 & 106 & 81 & 84 & 79 \\
\hline 20 & 20 & 20 & 20 & 22 & 22 & 22 & 24 & 20 \\
\hline 8 & 6 & 8 & 6 & 3 & 5 & 7 & 14 & 10 \\
\hline 204 & 211 & 210 & 185 & 189 & 173 & 189 & 186 & 179 \\
\hline 30 & 33 & 31 & 37 & 39 & 36 & 38 & 38 & 39 \\
\hline 93 & 94 & 87 & 121 & 123 & 123 & 129 & 123 & 124 \\
\hline$<1$ & 2 & 3 & 3 & 1 & 5 & 4 & 3 & 1 \\
\hline 34 & 24 & 39 & 30 & 31 & 42 & 34 & 34 & 29 \\
\hline 3 & 4 & 5 & 6 & 5 & 3 & 4 & 4 & 6 \\
\hline 18 & 16 & 15 & 17 & 16 & 17 & 19 & 15 & 19 \\
\hline 4 & 5 & 4 & 5 & 1 & 1 & 3 & 7 & $<1$ \\
\hline$<1$ & 2 & $<1$ & 2 & $<1$ & $<1$ & $<1$ & 3 & $<1$ \\
\hline - & 47 & 29 & 40 & 123 & 25 & 32 & 41 & 124 \\
\hline 81 & 79 & 83 & 82 & 79 & 80 & 77 & 77 & 78 \\
\hline 0.32 & 0.35 & 0.36 & 0.31 & 0.32 & 0.29 & 0.29 & 0.31 & 0.31 \\
\hline 0.19 & 0.17 & 0.17 & 0.14 & 0.13 & 0.14 & 0.15 & 0.12 & 0.15 \\
\hline 0.37 & 0.26 & 0.45 & 0.25 & 0.25 & 0.34 & 0.26 & 0.28 & 0.23 \\
\hline 1.47 & 1.19 & 1.19 & 1.13 & 1.01 & 1.16 & 1.23 & 0.97 & 1.20 \\
\hline 1.74 & 2.12 & 2.05 & 2.24 & 2.14 & 1.98 & 2.18 & 2.20 & 2.32 \\
\hline 440 & 506 & 495 & 457 & 800 & 525 & 381 & 225 & 387 \\
\hline 0.17 & 0.11 & 0.19 & 0.16 & 0.16 & 0.24 & 0.18 & 0.18 & 0.16 \\
\hline 0.0 & 0.2 & 0.0 & 0.2 & 0.0 & 0. & 0. & 0. & 0.0 \\
\hline 2.6 & , & 2.8 & 2.0 & 1.7 & 1.9 & 1.9 & 2.2 & 2.8 \\
\hline 22.8 & 20.3 & 22.9 & 22.8 & 24.0 & 27.5 & 26.1 & 26.5 & 23.4 \\
\hline 31.2 & 33.8 & 33.2 & 29.5 & 30.4 & 27.7 & 29.9 & 29.0 & 29.8 \\
\hline 0.0 & 0.0 & 0.0 & 0.0 & 0.0 & 0.0 & 0.0 & 0.0 & 0.0 \\
\hline 21.1 & 23.4 & 24.7 & 23.4 & 24.2 & 22.9 & 24.4 & 24.7 & 23.7 \\
\hline 16.4 & 14.5 & 7.6 & 15.5 & 13.0 & 9.4 & 8.2 & 6.1 & 12.1 \\
\hline 0.5 & 0.0 & 3.6 & 0.0 & 0.4 & 4.4 & 3.3 & 5.3 & 1.8 \\
\hline 1.7 & 1.8 & 1.7 & 1.9 & 1.8 & 1.8 & 1.8 & 1.8 & 1.9 \\
\hline 2.5 & 2.4 & 2.3 & 3.1 & 3.1 & 3.1 & 3.1 & 3.0 & 3.1 \\
\hline 0.4 & 0.5 & 0.4 & 0.6 & 0.6 & 0.4 & 0.5 & 0.5 & 0.5 \\
\hline
\end{tabular}

a Normative values based on $\mathrm{Fe}_{2} \mathrm{O}_{3} / \mathrm{FeO}$ ratio of $0.15 ; \mathrm{Fe}_{2} \mathrm{O}_{3}$ is total iron as $\mathrm{Fe}_{2} \mathrm{O}_{3}$.

$\mathrm{Ce}_{\mathrm{N}} / \mathrm{Y}_{\mathrm{N}}$ represents ratio of chondrite-normalized values.

plagioclase and pyroxene microlites, with granular or skeletal magnetite set in a cryptocrystalline matrix, often partially altered to clays. Chrome spinel is an accessory phase in units 3 and 4 .

Veins and fractures lined by calcite and clays are common throughout the recovered section, as are carbonate- and clay-filled amygdules. In unit 4 (Core 60 ), native copper occurs in a carbonate vein together with well-formed pyrite octahedra. Other veins in this unit are lined by a talc-like mineral which has also replaced the olivine phenocrysts. Olivine in other units is invariably partially or totally replaced by pseudo- morphs of clay and chloritic material, with occasional zeolites or calcite. In unit 2, the cores of some large plagioclase phenocrysts are saussuritized.

The basalts recovered at Site 443 (Table 6) are olivine- and hypersthene-normative sub-alkaline tholeiites; no nepheline- or quartz-normative varieties have been found (Figure 3). On the basis of their chemistry, the basalts have been divided into five geochemical units (Figure 17): units 443-1 and 443-2 correspond to lithologic unit 2; geochemical unit 443-3 corresponds to most of lithologic unit 3; geochemical unit 443-4 consists of the lower part of lithologic unit 3 , and lithologic units 4 


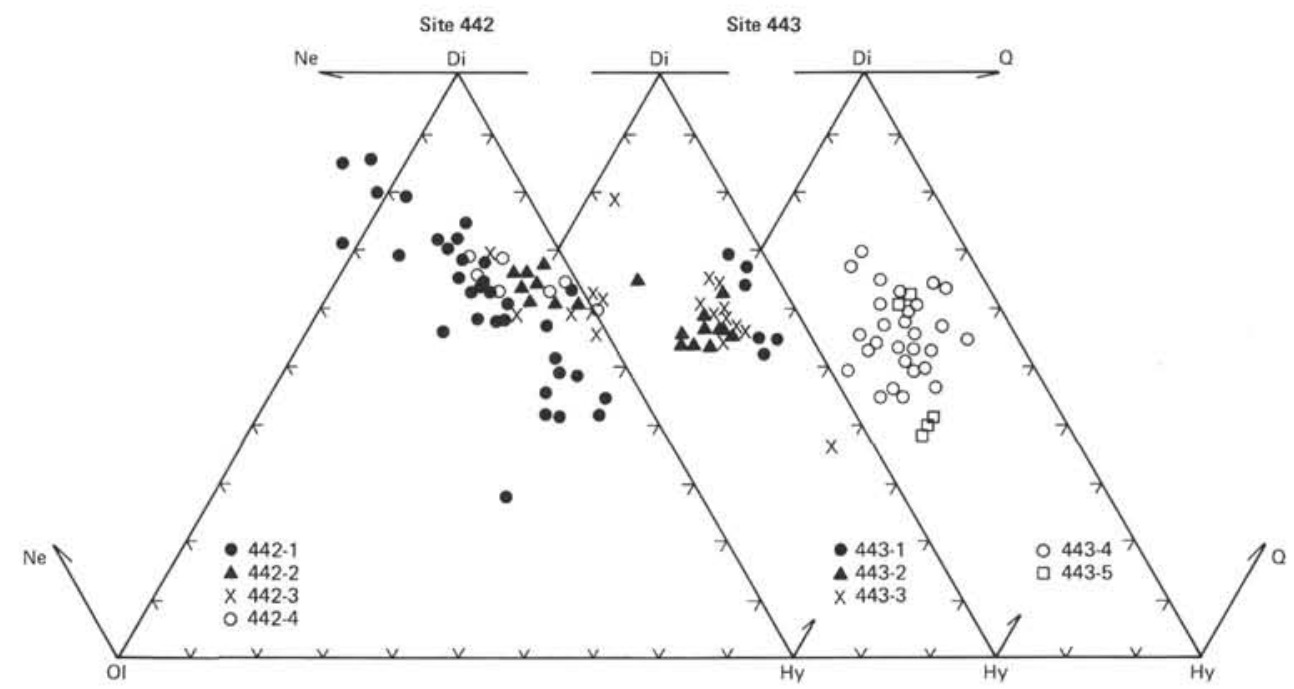

Figure 3. Normative nepheline ( $\mathrm{Ne})$, olivine $(\mathrm{Ol})$, diopside (Di), hypersthene (Hy), quartz $(Q)$ tetrahedra for basalts from Sites 442 and 443 . Normative values based on $\mathrm{Fe}_{2} \mathrm{O}_{3} / \mathrm{FeO}$ ratio of 0.15 .

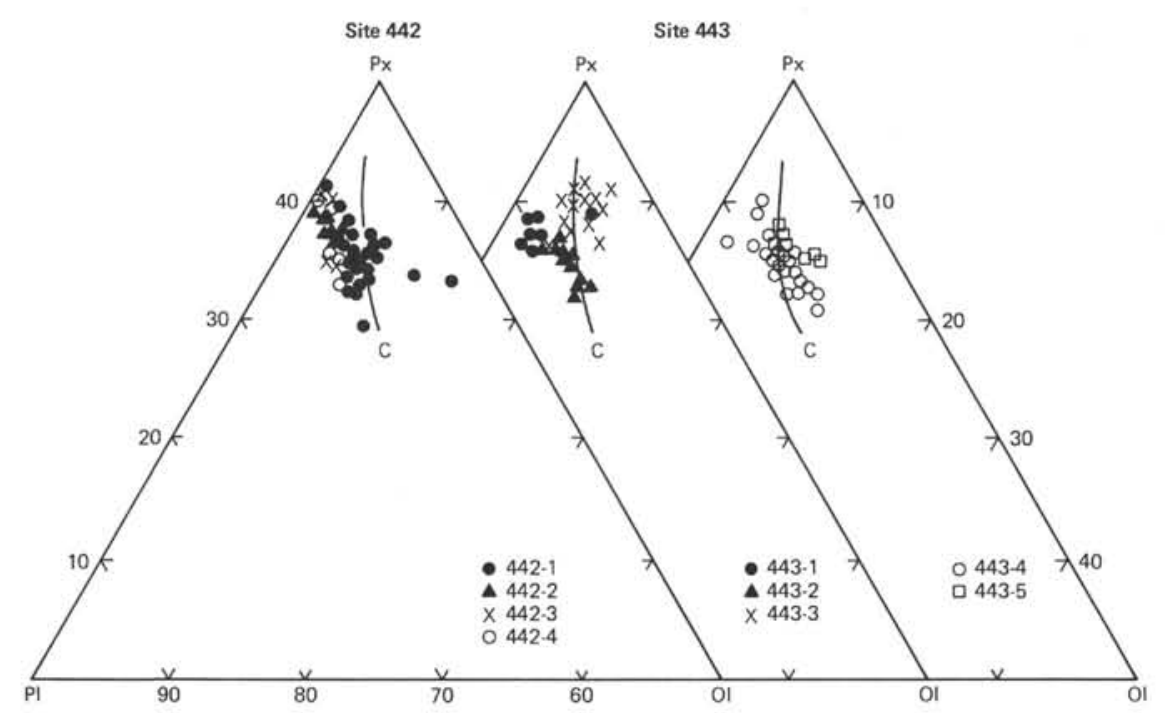

Figure 4. Normative plagioclase, pyroxene, olivine diagrams for basalts from Sites 442 and 443. The Fo-An cotectic (C) is from Shido et al. (1971).

and 5; and geochemical unit 443-5 corresponds to lithologic unit 6 . Geochemical unit 443-4 cannot be distinguished from 443-5 using the present XRF data, but because they are petrographically distinct and because the two units have different Th/Hf ratios (Wood et al., this volume) we have retained the division for the purpose of the present discussion.

On the diagram of normative plagioclase-pyroxeneolivine, the basalts range in composition from olivine tholeiites (443-3, 443-4 and 443-5), through olivine-plagioclase tholeiites straddling the 1-atmosphere Fo-An cotectic (443-2), to plagioclase tholeiites (443-1)(Figure 4). This division broadly corresponds with the observed phenocryst assemblages, although many of the basalts from Site 443 are only sparsely phyric or even aphyric.
Thus, the phenocryst assemblage of lithologic unit 2 is dominated by plagioclase, whereas in the lower lithologic units, olivine phenocrysts are more abundant.

As with the Site 442 basalts, there is considerable down-hole variation in chemistry at Site 443 (Figure 17). From the $\mathrm{Fe} / \mathrm{Mg}$ ratio and $\mathrm{Zr}, \mathrm{Cr}$, and $\mathrm{Ni}$ abundances (Figures 17 and 18), it is evident that geochemical units 443-4 and 443-5 contain the most-primitive basalts (with the lowest $\mathrm{Fe} / \mathrm{Mg}$ ratio and $\mathrm{Zr}$, and highest $\mathrm{Ni}$ and $\mathrm{Cr}$ ) and that units 443-1 and 443-3 contain the most-evolved basalts recovered at this site. Unit 443-2 occupies an intermediate position. However, there is considerable within-unit variation. Thus, unit 443-3 basalts contain 54 to $156 \mathrm{ppm} \mathrm{Ni}$, one sample (Core $58-3,29 \mathrm{~cm}$ ) containing $405 \mathrm{ppm} \mathrm{Ni}, 428 \mathrm{ppm} \mathrm{Cr}$, and 14.01 per cent 


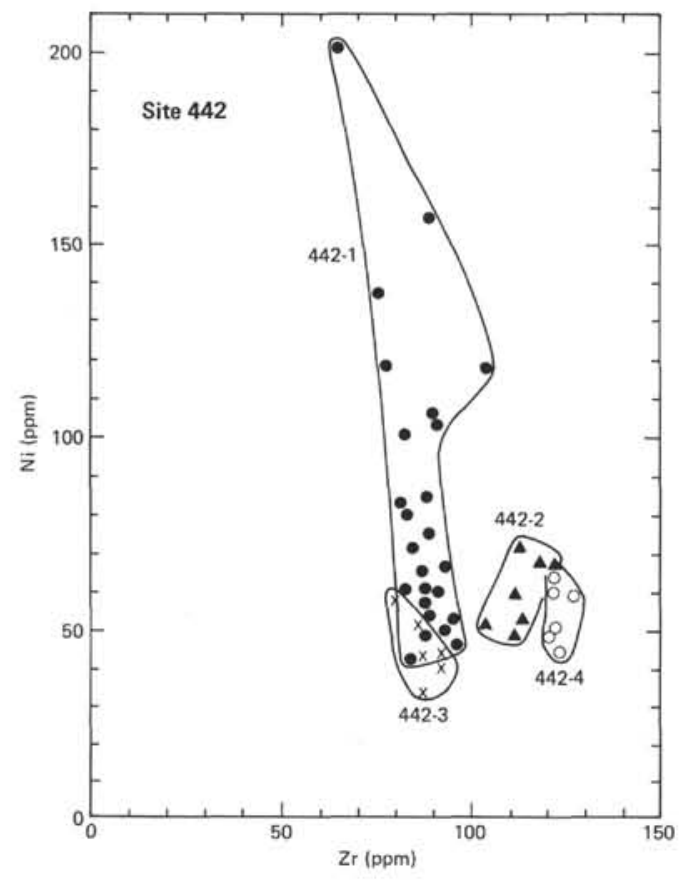

Figure 5. Ni versus $\mathrm{Zr}$, Site 442. The numerals indicate geochemical units in down-hole order (see Figure 2).

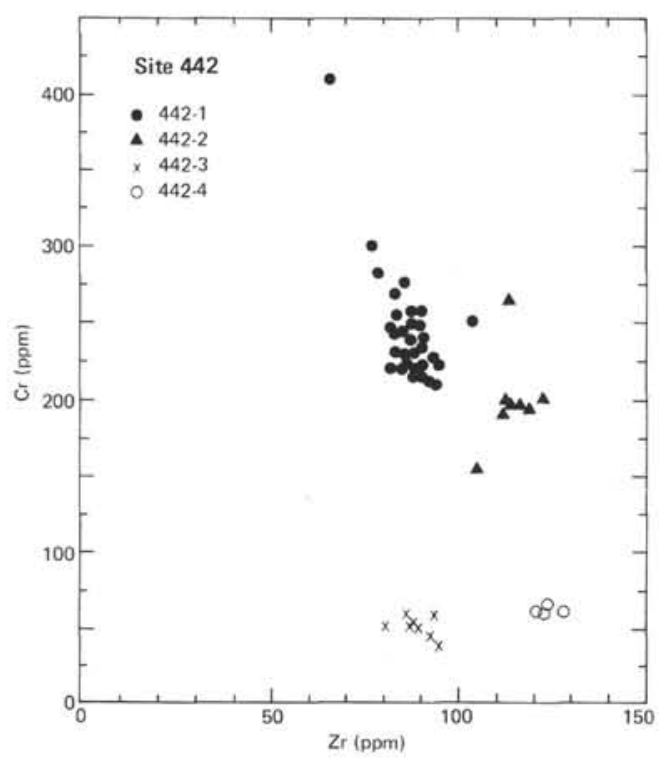

Figure 6. Cr versus $\mathrm{Zr}$, Site 442.

$\mathrm{MgO}$. $\mathrm{Ni}$ and $\mathrm{Cr}$ also show strong variations in Units 443-1, 443-4, and 443-5. Whereas fractional crystallization (involving removal of pyroxene, olivine, and (or) chrome spinel from the melt) may be responsible for some of the observed variations in $\mathrm{Ni}$ and $\mathrm{Cr}$, it is evident that some within-flow differentiation has also occurred, particularly in the thickest flows of lithologic unit 3. Thus, the high $\mathrm{Ni}$ and $\mathrm{Cr}$ levels observed in 58-3, $29 \mathrm{~cm}$ are probably due to the presence of cumulus olivine and chrome spinel. However, the olivine accumula-

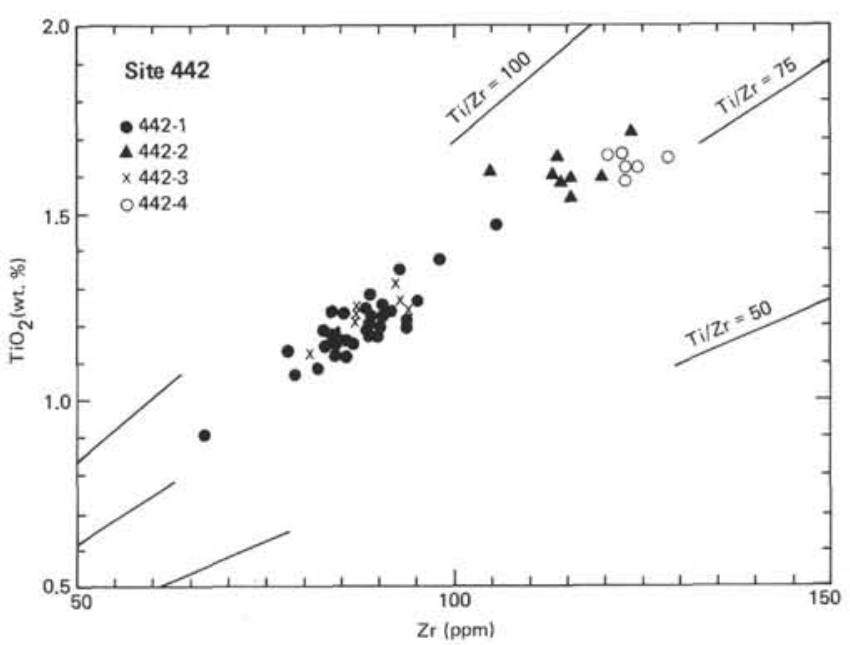

Figure 7. $\mathrm{TiO}_{2}$ versus $\mathrm{Zr}$, Site 442 .

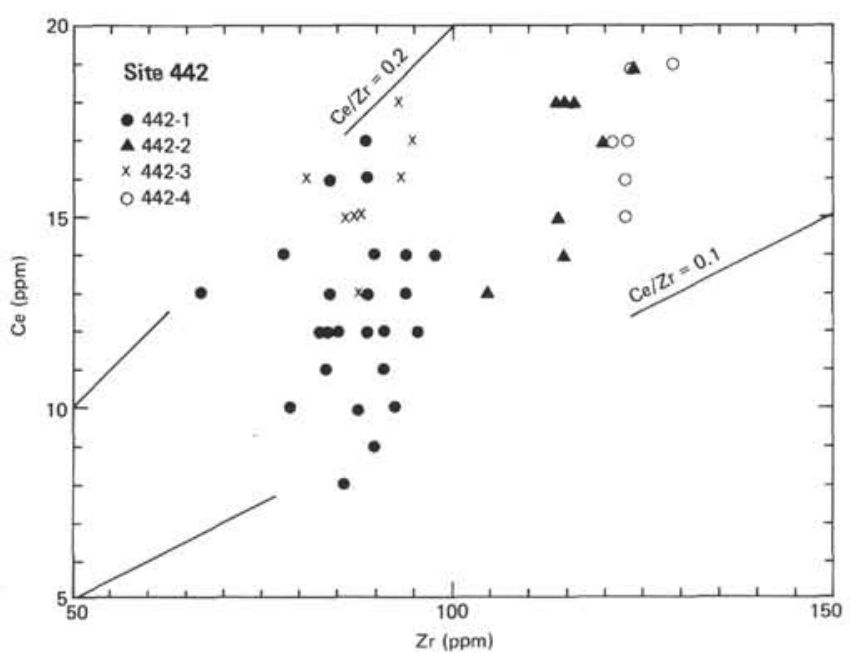

Figure 8. Ce versus $\mathrm{Zr}$, Site 442.

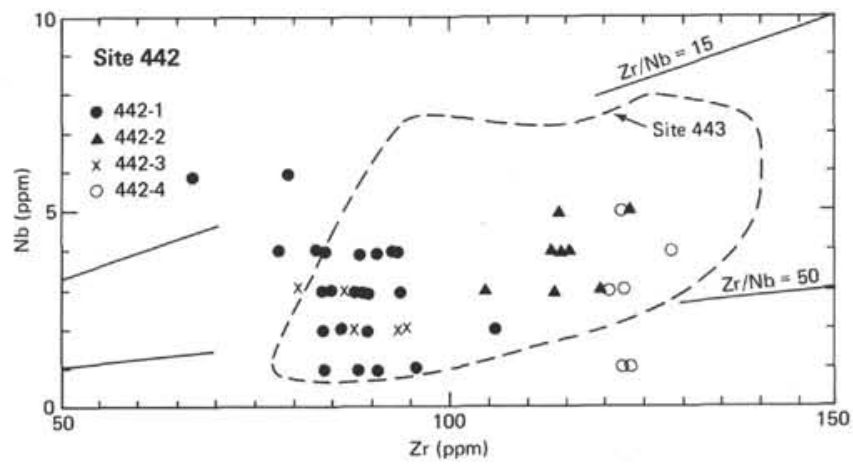

Figure 9. $\mathrm{Nb}$ versus $\mathrm{Zr}$, Sites 442 and 443. Individual data points for Site 443 omitted for clarity.

tion which was observed petrographically in unit $2 \mathrm{E}$ has not been geochemically confirmed. In general, it is apparent that the basalts from Site 443 have undergone 


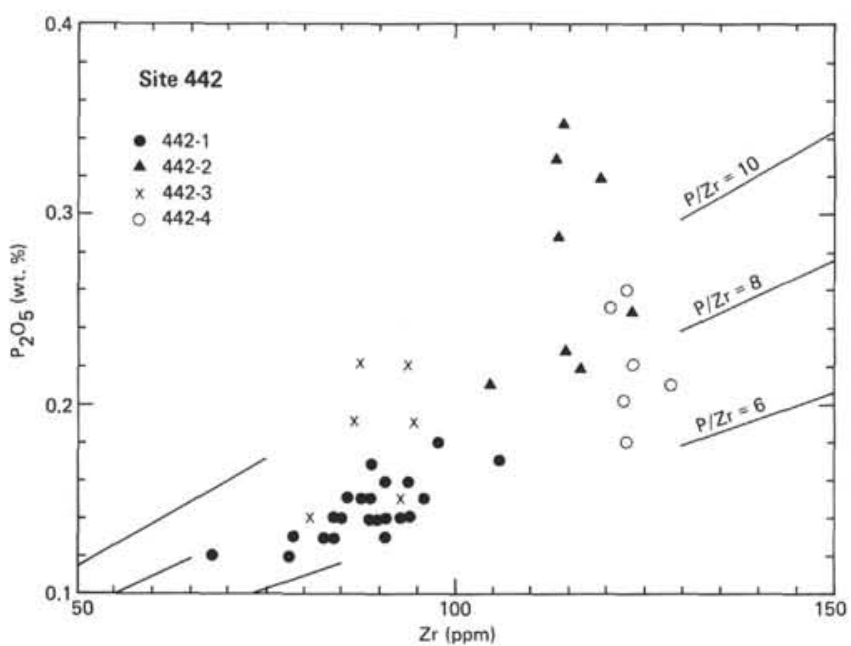

Figure 10. $\mathrm{P}_{2} \mathrm{O}_{5}$ versus $\mathrm{Zr}$, Site 442. The scatter of data points along the $\mathrm{P}_{2} \mathrm{O}_{5}$ axis in geochemical units 2, 3 and 4 appears to be a function of alteration of these basalts. See also Figure 15.

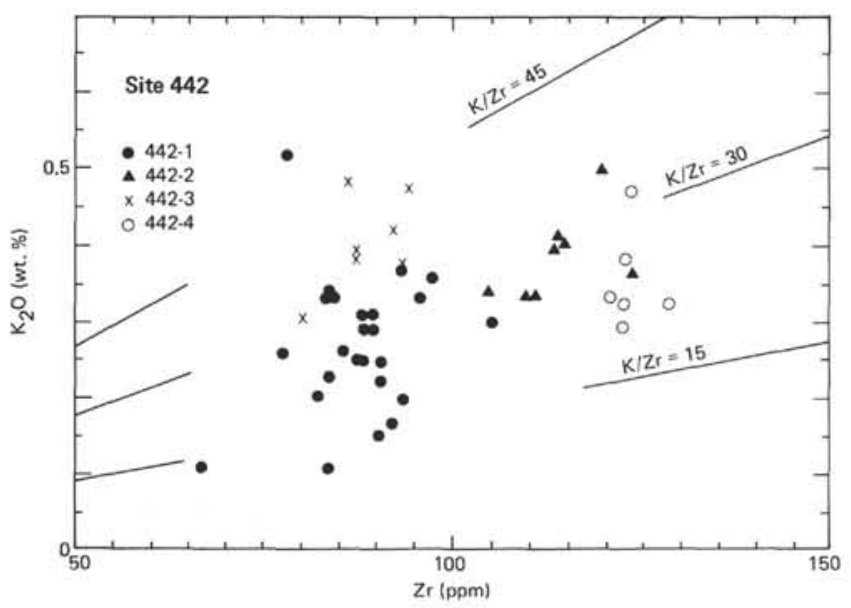

Figure 11. $\mathrm{K}_{2} \mathrm{O}$ versus $\mathrm{Zr}$, Site 442. Scatter of data points is indicative of sea-water alteration.

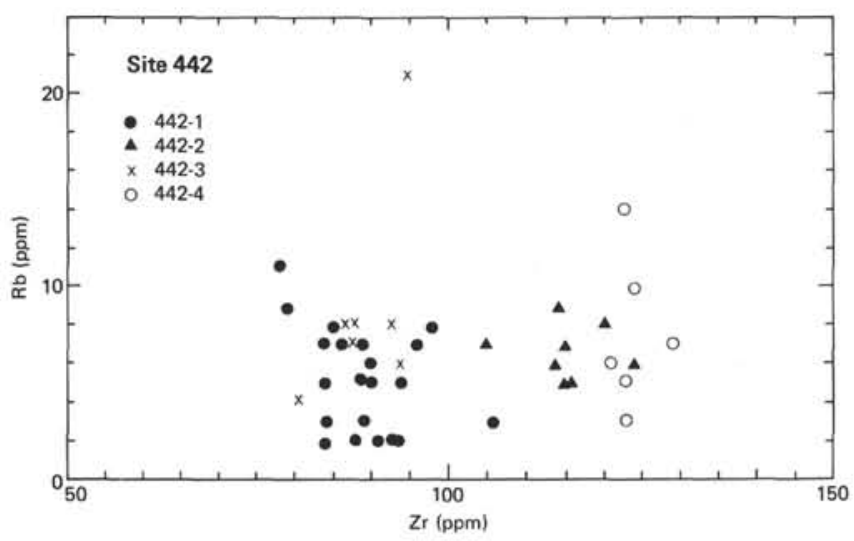

Figure 12. Rb versus $Z r$, Site 442 . Scatter of data points along $R b$ axis in geochemical units 3 and 4 is indicative of sea-water alteration.

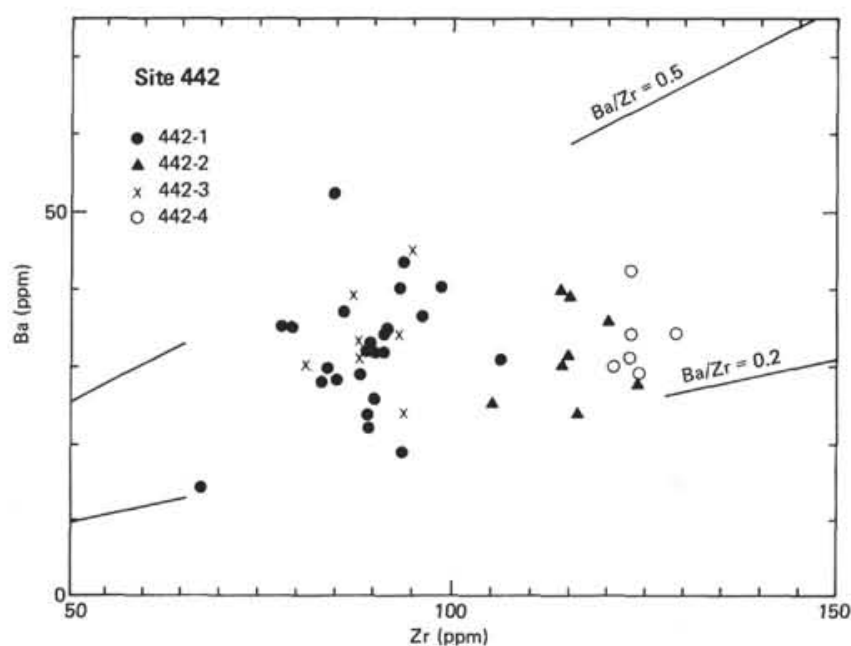

Figure 13. Ba versus $\mathrm{Zr}$, Site 442. As in figures 11 and 12, scatter of data points may be a result of sea-water alteration.

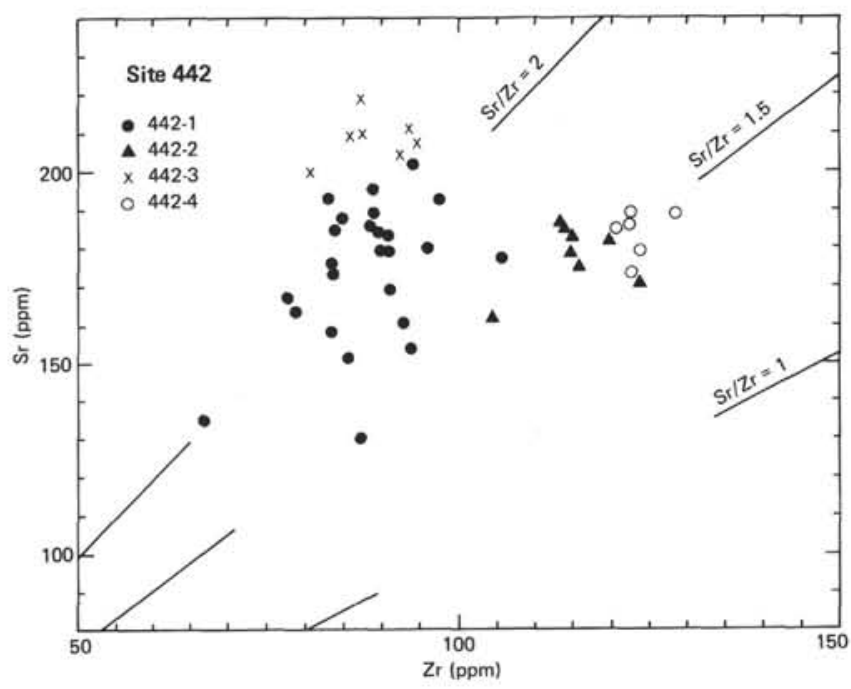

Figure 14. Sr versus $\mathrm{Zr}$, Site 442.

considerable fractionation prior to their eruption: their $\mathrm{Ni}$ and $\mathrm{Cr}$ contents are too low, and $\mathrm{Fe} / \mathrm{Mg}$ ratios too high, for the liquids to have been in equilibrium with upper-mantle olivine compositions $\left(\mathrm{Fo}_{88-90}\right.$; Green, 1970; O’Hara, 1973).

Using $\mathrm{Zr}$ as an index of fractionation, hygromagmatophile trace elements have been plotted in Figures 19 to 25. $\mathrm{TiO}_{2}, \mathrm{P}_{2} \mathrm{O}_{5}, \mathrm{Ce}, \mathrm{La}, \mathrm{Nb}$, and $\mathrm{Y}$ all show strong positive correlations with $\mathrm{Zr}$, similar to the Site $\mathbf{4 4 2}$ data. In general, the ratios of these elements between the different geochemical units are constant, particularly for the more-HYG elements $(\mathrm{Ce}, \mathrm{La}, \mathrm{Nb}$, and $\mathrm{Zr})$. The plot of $\mathrm{Zr}$ versus $\mathrm{Y}$ (Figure 20), however, suggests that geochemical units 443-2 and 443-5 have slightly higher $\mathrm{Y} / \mathrm{Zr}$ ratios than units $443-3$ and $443-1$; unit 443-4 shows a range of $\mathrm{Y} / \mathrm{Zr}$ ratios covering both extremes. A strong correlation exists between $\mathrm{P}_{2} \mathrm{O}_{5}$ and $\mathrm{Zr}$ (Figure 21): the incoherent scatter of $\mathrm{P}_{2} \mathrm{O}_{5}$ in lithologic unit 2 at Site 442 is not observed at Site 443 . 


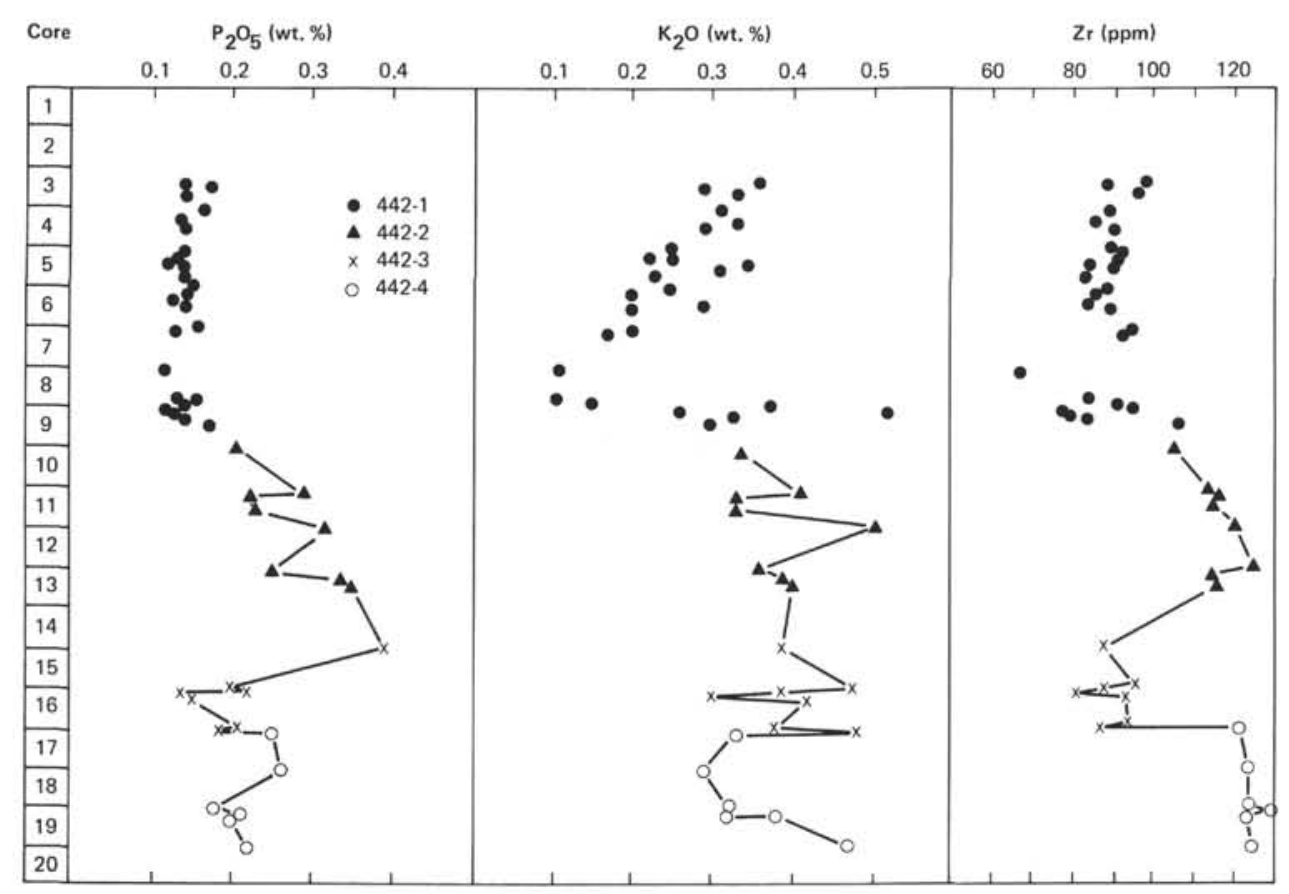

Figure 15. Down-hole variation at Site 442 in the abundancies of $\mathrm{P}_{2} \mathrm{O}_{5}, \mathrm{~K}_{2} \mathrm{O}$, and $\mathrm{Zr}$. $\mathrm{P}_{2} \mathrm{O}_{5}$ and $\mathrm{Zr}$ show a broad correlation in unit 442-1 but the distribution of $\mathrm{P}_{2} \mathrm{O}_{5}$ is incoherent in units 2, 3 and $4 . \mathrm{K}_{2} \mathrm{O}$ also exhibits considerable scatter, probably indicative of sea-water alteration.

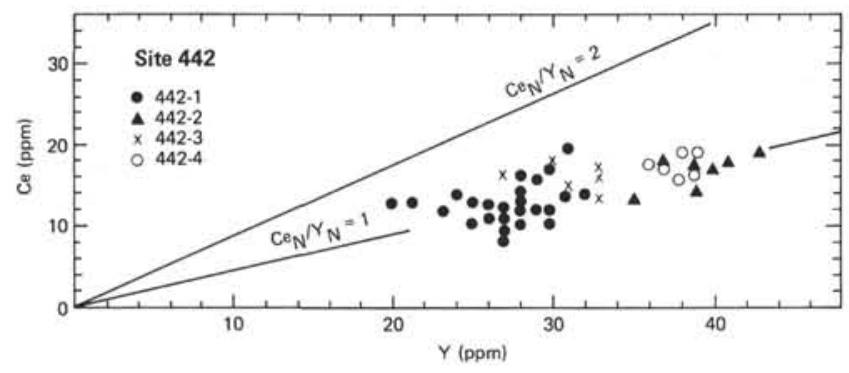

Figure 16. Ce versus $Y$, Site 442. $C e_{N} / Y_{N}$ indicates chondrite normalized $\mathrm{Ce} / Y$ ratios (approximately equivalent to $\mathrm{Ce}_{\mathrm{N}} / \mathrm{Ho}_{\mathrm{N}}$ ratio).

$\mathrm{K}_{2} \mathrm{O}, \mathrm{Rb}$, and $\mathrm{Sr}$ do not show clear correlations with $\mathrm{Zr}$ (Figures 22 and 23) and, as with the Site 442 basalts, we suspect that this is essentially because of alteration by sea water. The least-altered samples show low $\mathrm{K} / \mathrm{Zr}$, $\mathrm{Ba} / \mathrm{Zr}$, and $\mathrm{Rb} / \mathrm{Zr}$ ratios, similar to those of $\mathrm{N}$-type ocean-ridge basalts (Table 4; Erlank and Kable, 1976).

$\mathrm{Ce}_{\mathrm{N}} / \mathrm{Y}_{\mathrm{N}}$ ratios for the basalts of Site 443 are, within the error of XRF analysis at such low levels of Ce, constant throughout the five units, at about 1 (Figure 26). This is in agreement with preliminary, more-comprehensive REE data which indicate that the $\mathrm{Ce}_{\mathrm{N}} / \mathrm{Yb}_{\mathrm{N}}$ ratio is approximately 1.5 in unit 443-3, and 1.2 in unit 443-4 (Wood et al., this volume; unpublished data of the authors). The $\mathrm{Ce}_{\mathrm{N}} / \mathrm{Y}_{\mathrm{N}}$ ratios for the Site 443 and 442 basalts are practically indistinguishable, and are similar to the low values reported for N-type ocean-ridge basalts (e.g., Kay et al., 1970; Sun et al., 1979). Other trace-element ratios, in addition to the $\mathrm{K} / \mathrm{Zr}, \mathrm{Ba} / \mathrm{Zr}$, and $\mathrm{Rb} / \mathrm{Zr}$ ratios already mentioned, indicate close similarities between the Site 443, 442, and N-type oceanridge basalts. $\mathrm{Zr} / \mathrm{Nb}$ ratios are high (25-40; Figure 9), $\mathrm{Ce} / \mathrm{Zr}$ ratios are low (Figure 26 ), and $\mathrm{Y} / \mathrm{Zr}$ ratios approach chondritic values (Figure 20). However, neither the Site 442 nor Site 443 basalts show the strong depletion of all more-HYG elements that is observed in $\mathrm{N}$-type basalts. Thus, $\mathrm{Ce}_{\mathrm{N}} / \mathrm{Y}_{\mathrm{N}}$ is approximately 1 , and not significantly less than 1 , as in $\mathrm{N}$-type ocean-ridge basalts (Sun et al., 1979).

With the exception of the slight variation in $\mathrm{Y} / \mathrm{Zr}$ ratio, the constant ratios of most hygromagmatophile elements among the units of Site 443 indicate that most of the basalts could be derived from a common basaltic parent by varying degrees of low-pressure fractional crystallization of olivine, plagioclase, and (or) chrome spinel. The rapid decrease in $\mathrm{Ni}$ and $\mathrm{Cr}$ relative to $\mathrm{Zr}$ (Figure 18) supports this suggestion. However, to vary the $\mathrm{Y} / \mathrm{Zr}$ ratio (and the $\mathrm{Th} / \mathrm{Hf}$ ratio; Wood et al., this volume) requires either variable degrees of partial melting, or open-system crystal fractionation (O'Hara, 1977), in order to retain $\mathrm{Y}$ in the refractory mineral assemblage. We suggest, judging from $\mathrm{Y}$ and $\mathrm{Zr}$ distributions in basalts recovered on DSDP Leg 49 in the North Atlantic Ocean (Tarney et al., 1978), that units 443-1 and 443-3 represent magma batches derived by smaller degrees of partial melting of the mantle source, and that units 443-2 and 443-5 represent slightly higher degrees of partial melting of the same mantle source. Unit 443-4 occupies an intermediate position, and it is apparent that most of the units subsequently underwent con- 
TABLE 4

Representative Analyses and Element Ratios of Basalts from the Daito Basin and from the Atlantic Ocean ${ }^{\mathrm{a}}$

\begin{tabular}{|c|c|c|c|c|c|c|c|c|c|c|c|c|}
\hline \multirow[b]{2}{*}{ Component } & \multicolumn{4}{|c|}{$\begin{array}{l}\text { Daito Basin, } \\
\text { Alkalic Group }\end{array}$} & \multicolumn{4}{|c|}{$\begin{array}{l}\text { Daito Basin, } \\
\text { Tholeiitic Group }\end{array}$} & \multicolumn{4}{|c|}{ Atlantic Ocean Basalts } \\
\hline & $446-1$ & $446-4$ & $446-5$ & $446-10 \mathrm{a}$ & $446-6$ & $446-8$ & $446-9$ & $446-14$ & 1 & 2 & 3 & 4 \\
\hline $\mathrm{SiO}_{2}$ & 45.8 & 44.8 & 42.4 & 47.1 & 49.6 & 47.8 & 46.3 & 49.6 & 50.01 & 49.30 & 47.63 & 46.45 \\
\hline $\mathrm{TiO}_{2}^{2}$ & 3.67 & 4.9 & 4.07 & 3.72 & 3.61 & 4.16 & 4.95 & 3.17 & 1.37 & 1.74 & 5.22 & 2.94 \\
\hline $\mathrm{Al}_{2} \mathrm{O}_{3}$ & 14.7 & 13.3 & 10.3 & 14.9 & 13.0 & 13.1 & 13.7 & 13.3 & 16.18 & 14.01 & 12.54 & 16.66 \\
\hline $\mathrm{Fe}_{2} \mathrm{O}_{3}$ & 10.88 & 11.85 & 12.77 & 9.76 & 13.01 & 14.49 & 14.97 & 12.53 & 10.18 & 12.13 & 16.71 & 11.00 \\
\hline $\mathrm{MnO}$ & 0.36 & 0.19 & 0.22 & 0.24 & 0.17 & 0.21 & 0.23 & 0.23 & - & 0.19 & 0.23 & 0.07 \\
\hline $\mathrm{MgO}$ & 4.80 & 6.25 & 12.43 & 4.97 & 6.33 & 5.82 & 5.56 & 6.19 & 7.71 & 8.09 & 5.61 & 5.30 \\
\hline $\mathrm{CaO}$ & 11.14 & 12.44 & 11.25 & 11.53 & 9.88 & 10.16 & 8.30 & 10.86 & 11.33 & 11.65 & 9.23 & 10.38 \\
\hline $\mathrm{Na}_{2} \mathrm{O}$ & 3.16 & 2.81 & 1.49 & 2.97 & 2.69 & 2.50 & 3.07 & 2.56 & 2.79 & 2.30 & 3.04 & 3.75 \\
\hline $\mathrm{K}_{2} \mathrm{O}$ & 2.04 & 1.55 & 0.74 & 1.95 & 0.27 & 0.20 & 1.03 & 0.28 & 0.22 & 0.18 & 0.83 & 1.24 \\
\hline $\mathrm{P}_{2} \mathrm{O}_{5}$ & 0.37 & 0.99 & 0.73 & 0.35 & 0.34 & 0.43 & 0.51 & 0.37 & 0.13 & 0.22 & 0.56 & 0.48 \\
\hline L.O.I. & 2.53 & 0.48 & 2.88 & 2.03 & 1.07 & 0.94 & 1.11 & 1.09 & 0.87 & 0.83 & n.d. & 1.32 \\
\hline Total & 99.40 & 99.53 & 99.33 & 99.45 & 100.02 & 99.86 & 99.70 & 100.08 & 100.79 & 100.64 & 101.6 & 99.59 \\
\hline $\mathrm{Ni}$ & 29 & 45 & 293 & 28 & 72 & 33 & 20 & 94 & 100 & 106 & 15 & 9 \\
\hline $\mathrm{Cr}$ & 18 & 69 & 1137 & 30 & 141 & 23 & 3 & 175 & 200 & 317 & 66 & 70 \\
\hline $\mathrm{Rb}$ & 36 & 22 & 16 & 30 & 1 & 2 & 9 & 11 & 1 & 4 & 16 & 22 \\
\hline $\mathrm{Sr}$ & 689 & 1088 & 480 & 756 & 367 & 447 & 453 & 426 & 124 & 103 & 445 & 693 \\
\hline Y & 27 & 26 & 19 & 28 & 31 & 37 & 41 & 33 & 33 & 35 & 36 & - \\
\hline $\mathrm{Zr}$ & 206 & 188 & 150 & 211 & 194 & 302 & 378 & 229 & 90 & 110 & 290 & 220 \\
\hline $\mathrm{Nb}$ & 27 & 32 & 24 & 25 & 19 & 28 & 32 & 24 & 3 & 13 & 31 & - \\
\hline $\mathrm{Ba}$ & 313 & 362 & 212 & 332 & 121 & 143 & 225 & 102 & 12 & 70 & 180 & 302 \\
\hline $\mathrm{La}$ & 22 & 25 & 22 & 24 & 18 & 32 & 34 & 26 & 3 & 6 & 28 & 29 \\
\hline $\mathrm{Ce}$ & 45 & 58 & 42 & 48 & 42 & 60 & 70 & 50 & 9 & 19 & 57 & 51 \\
\hline $\mathrm{Fe} / \mathrm{Mg}$ & 2.63 & 2.20 & 1.19 & 2.28 & 2.38 & 2.89 & 3.12 & 2.35 & 1.53 & 1.74 & 3.46 & 2.41 \\
\hline $\mathrm{Zr} / \mathrm{Nb}$ & 8 & 6 & 6 & 8 & 10 & 11 & 12 & 10 & 30 & 8 & 9 & - \\
\hline $\mathrm{Ce} / \mathrm{Zr}$ & 0.22 & 0.31 & 0.28 & 0.23 & 0.22 & 0.20 & 0.19 & 0.22 & 0.10 & 0.17 & 0.20 & 0.23 \\
\hline $\mathrm{Ce}_{\mathrm{N}} / \mathrm{Y}_{\mathrm{N}}$ & 4.09 & 5.48 & 5.43 & 4.21 & 3.33 & 3.98 & 4.19 & 3.72 & 0.67 & 1.33 & 3.90 & - \\
\hline $\mathrm{K} / \mathrm{Rb}$ & 470 & 586 & 383 & 539 & 2217 & 822 & 949 & 209 & 1826 & 374 & 431 & 468 \\
\hline $\mathrm{Ba} / \mathrm{Sr}$ & 0.45 & 0.33 & 0.44 & 0.44 & 0.33 & 0.32 & 0.50 & 0.24 & 0.10 & 0.68 & 0.40 & 0.44 \\
\hline $\mathrm{P} / \mathrm{Zr}$ & 7.8 & 23.0 & 21.2 & 7.2 & 7.7 & 6.2 & 5.9 & 7.1 & 6.3 & 8.7 & 8.4 & 9.5 \\
\hline $\mathrm{Ba} / \mathrm{Zr}$ & 1.52 & 1.93 & 1.41 & 1.57 & 0.62 & 0.47 & 0.60 & 0.45 & 0.13 & 0.64 & 0.62 & 1.37 \\
\hline
\end{tabular}

aAnalyses of Daito Basin basalts from Tables 6 and 7. Atlantic Ocean basalts: 1, typical "depleted" N-type ocean-ridge basalt; major elements from Engel et al. (1965); trace elements from Sun et al. (1979) and Bougault et al. (1979), with typical values for $\mathrm{Ni}$ and $\mathrm{Cr}$; 2 , transitional or "T-type" ocean-ridge basalt, Reykjanes Ridge, Leg 49, Site 409, unit 3 (Wood et al., 1979); 3, "enriched" ferrobasalt, Eldgjá, Iceland, sample ISL 79 (Wood et al., in press); 4, "enriched" alkali basalt, Fayal, Azores, sample FA47 (Joron et al., in prep.). Fe $2 \mathrm{O}_{3}$ is total iron as $\mathrm{Fe}_{2} \mathrm{O}_{3}$. $\mathrm{Ce}_{\mathrm{N}} / \mathrm{Y}_{\mathrm{N}}$ represents ratio of chondrite-normalized values.

siderable fractional crystallization (particularly units 443-3 and 443-1) prior to eruption.

\section{Site 444}

Site 444 (Figure 1) lies approximately $100 \mathrm{~km}$ south of Site 443, and along the same depositional, structural, and topographic trends. Located at $28^{\circ} 38.25^{\prime} \mathrm{N}, 137^{\circ}$ $41.03^{\prime} \mathrm{E}$, in 4843 meters of water, Hole $444 \mathrm{~A}$ drilled two basaltic units, some 50 meters thick, and 285 meters of sediments (Figure 27). The sediments above and between the two basaltic units are approximately $15 \mathrm{~m} . \mathrm{y}$. old, and Waples (this volume), judging from changes in the sedimentary organic chemistry profile, has suggested that the basalt of unit 1 was emplaced as a sill some $13 \mathrm{~m} . \mathrm{y}$. ago. In addition, the sediment immediately above unit 2 appears to have undergone thermal alteration, suggesting sub-sediment emplacement of unit 2 also. Hence, it appears that both units were emplaced as sills and are not true basement, which, from magneticanomaly data, has been tentatively dated as 21 to 22 m.y. at this site.
Unit 1 is an aphyric dolerite sill with petrography distinct from the other rocks recovered from the Shikoku Basin. The principal constituents are plagioclase $\left(\mathrm{An}_{60-82}\right)$ and augite; olivine varies from less than 1 per cent at the top of the sill to 15 per cent in the middle and lower sections. The groundmass is essentially replaced by cryptocrystalline chloritic or clayey matrix. Opaque minerals include magnetite and elongate, saw-toothed aggregates of ilmenite. Apatite needles are found as occasional inclusions in plagioclase, and chrome spinel occurs as inclusions in some relict olivines. Small, green-brown, platey crystals have been tentatively identified as kaersutite amphibole (Dick et al., this volume). The interval $20-1,53-81 \mathrm{~cm}$ is distinct from the remainder of the unit in containing less pyroxene (which has a strong pink-brown color indicative of a high $\mathrm{TiO}_{2}$ component), more magnetite and ilmenite, and aggregates of kaersutite up to $1 \mathrm{~cm}$ across. Although the very altered nature of the groundmass in this section suggests that the different mineralogy may be due to alteration, latestage magmatic differentiation may also have produced the observed mineralogy. In fact, the chemistry sup- 
TABLE 5

Representative Analyses and Element Ratios of Basalts from the Shikoku, East Scotia Sea, and Bransfield Strait Back-Arc Basins ${ }^{\mathrm{a}}$

\begin{tabular}{|c|c|c|c|c|c|c|c|c|c|c|}
\hline \multirow[b]{2}{*}{ Component } & \multicolumn{6}{|c|}{ Shikoku Basin } & \multicolumn{2}{|c|}{ East Scotia Sea } & \multicolumn{2}{|c|}{ Bransfield Strait } \\
\hline & $442-1$ & $442-3$ & $443-3$ & $443-4$ & $444-1$ & $444-2$ & D 20.43B & D24.14 & B138.2 & P $640.1 b$ \\
\hline $\mathrm{SiO}_{2}$ & 50.2 & 50.2 & 50.3 & 49.7 & 50.3 & 50.2 & 50.7 & 53.8 & 51.9 & 52.9 \\
\hline $\mathrm{TiO}_{2}^{-}$ & 1.26 & 1.13 & 1.83 & 1.22 & 1.94 & 1.61 & 1.29 & 0.61 & 1.5 & 0.64 \\
\hline $\mathrm{Al}_{2} \mathrm{O}_{3}$ & 15.9 & 16.3 & 15.5 & 16.3 & 16.2 & 16.1 & 16.6 & 14.5 & 16.2 & 17.7 \\
\hline $\mathrm{Fe}_{2} \mathrm{O}_{3}$ & 8.66 & 8.61 & 9.42 & 9.13 & 7.96 & 10.15 & 8.4 & 9.2 & 9.46 & 7.43 \\
\hline $\mathrm{MnO}$ & 0.10 & 0.12 & 0.10 & 0.13 & 0.15 & 0.17 & 0.16 & 0.17 & 0.18 & 0.13 \\
\hline $\mathrm{MgO}$ & 7.93 & 6.80 & 7.52 & 7.95 & 6.25 & 6.87 & 7.67 & 7.71 & 6.11 & 6.14 \\
\hline $\mathrm{CaO}$ & 11.11 & 12.17 & 10.69 & 11.62 & 8.59 & 11.36 & 11.12 & 10.80 & 10.07 & 10.30 \\
\hline $\mathrm{Na}_{2} \mathrm{O}$ & 2.80 & 2.58 & 3.09 & 2.71 & 3.86 & 2.82 & 3.15 & 1.79 & 4.07 & 3.54 \\
\hline $\mathrm{K}_{2} \mathrm{O}$ & 0.22 & 0.30 & 0.22 & 0.10 & 2.31 & 0.16 & 0.34 & 0.24 & 0.28 & 0.47 \\
\hline $\mathrm{P}_{2} \mathrm{O}_{5}$ & 0.13 & 0.14 & 0.18 & 0.11 & 0.39 & 0.23 & 0.19 & 0.08 & 0.21 & 0.06 \\
\hline Total & 98.37 & 98.31 & 98.83 & 98.99 & 97.92 & 99.73 & 99.63 & 98.99 & 99.96 & 99.27 \\
\hline $\mathrm{Ni}$ & 157 & 59 & 73 & 113 & 26 & 53 & 63 & 42 & 35 & 40 \\
\hline $\mathrm{Cr}$ & 232 & 50 & 225 & 297 & 127 & 245 & 270 & 295 & 141 & 130 \\
\hline $\mathrm{Rb}$ & 2 & 4 & 3 & 1 & 16 & 2 & 5 & 4 & 3 & 11 \\
\hline $\mathrm{Sr}$ & 179 & 200 & 178 & 154 & 391 & 160 & 193 & 123 & 340 & 332 \\
\hline $\mathrm{Y}$ & 27 & 27 & 40 & 28 & 38 & 40 & 29 & 14 & 26 & 10 \\
\hline $\mathrm{Zr}$ & 91 & 81 & 126 & 87 & 209 & 141 & 107 & 40 & 144 & 58 \\
\hline $\mathrm{Nb}$ & 4 & 3 & 7 & 3 & 31 & 7 & 3 & $<1$ & 2 & $<1$ \\
\hline $\mathrm{Ba}$ & 35 & 30 & 50 & 31 & 177 & 71 & 49 & 55 & 88 & 70 \\
\hline $\mathrm{La}$ & 2 & 4 & 7 & 4 & 18 & 8 & - & - & 8 & 2 \\
\hline $\mathrm{Ce}$ & 11 & 16 & 17 & 8 & 42 & 20 & 16.1 & 6.45 & 22 & 8 \\
\hline $\mathrm{Fe} / \mathrm{Mg}$ & 1.27 & 1.47 & 1.45 & 1.33 & 1.48 & 1.71 & 1.27 & 1.38 & 1.80 & 1.40 \\
\hline $\mathrm{Zr} / \mathrm{Nb}$ & 23 & 27 & 18 & 29 & 7 & 20 & 36 & $>40$ & 72 & $>58$ \\
\hline $\mathrm{Ce} / \mathrm{Zr}$ & 0.12 & 0.20 & 0.13 & 0.09 & 0.20 & 0.14 & 0.15 & 0.16 & 0.15 & 0.14 \\
\hline $\mathrm{Ce}_{\mathrm{N}} / \mathrm{Y}_{\mathrm{N}}$ & 1.0 & 1.46 & 1.04 & 0.70 & 2.71 & 1.23 & 1.37 & 1.13 & 2.08 & 0.97 \\
\hline $\mathrm{K} / \mathrm{Rb}$ & 897 & 623 & 595 & 855 & 1198 & 664 & 564 & 498 & 775 & 355 \\
\hline $\mathrm{Ba} / \mathrm{Sr}$ & 0.20 & 0.15 & 0.28 & 0.20 & 0.45 & 0.44 & 0.25 & 0.45 & 0.26 & 0.21 \\
\hline $\mathrm{P} / \mathrm{Zr}$ & 6.2 & 7.5 & 6.2 & 5.5 & 8.1 & 7.1 & 7.8 & 8.7 & 6.4 & 4.5 \\
\hline $\mathrm{Ba} / \mathrm{Zr}$ & 0.38 & 0.37 & 0.40 & 0.36 & 0.85 & 0.50 & 0.46 & 1.38 & 0.61 & 1.21 \\
\hline
\end{tabular}

aAnalyses of shikoku Basin basalts taken from Tables 2 to 5. Analyses of East Scotia Sea basalts from Saunders and Tarney (1979), those for Bransfield Strait basalts from Weaver et al. (1979). $\mathrm{Fe}_{2} \mathrm{O}_{3}$ is total iron as $\mathrm{Fe}_{2} \mathrm{O}_{3}$. $\mathrm{Ce}_{\mathrm{N}} / \mathrm{Y}_{\mathrm{N}}$ represents ratio of chondrite-normalized values.

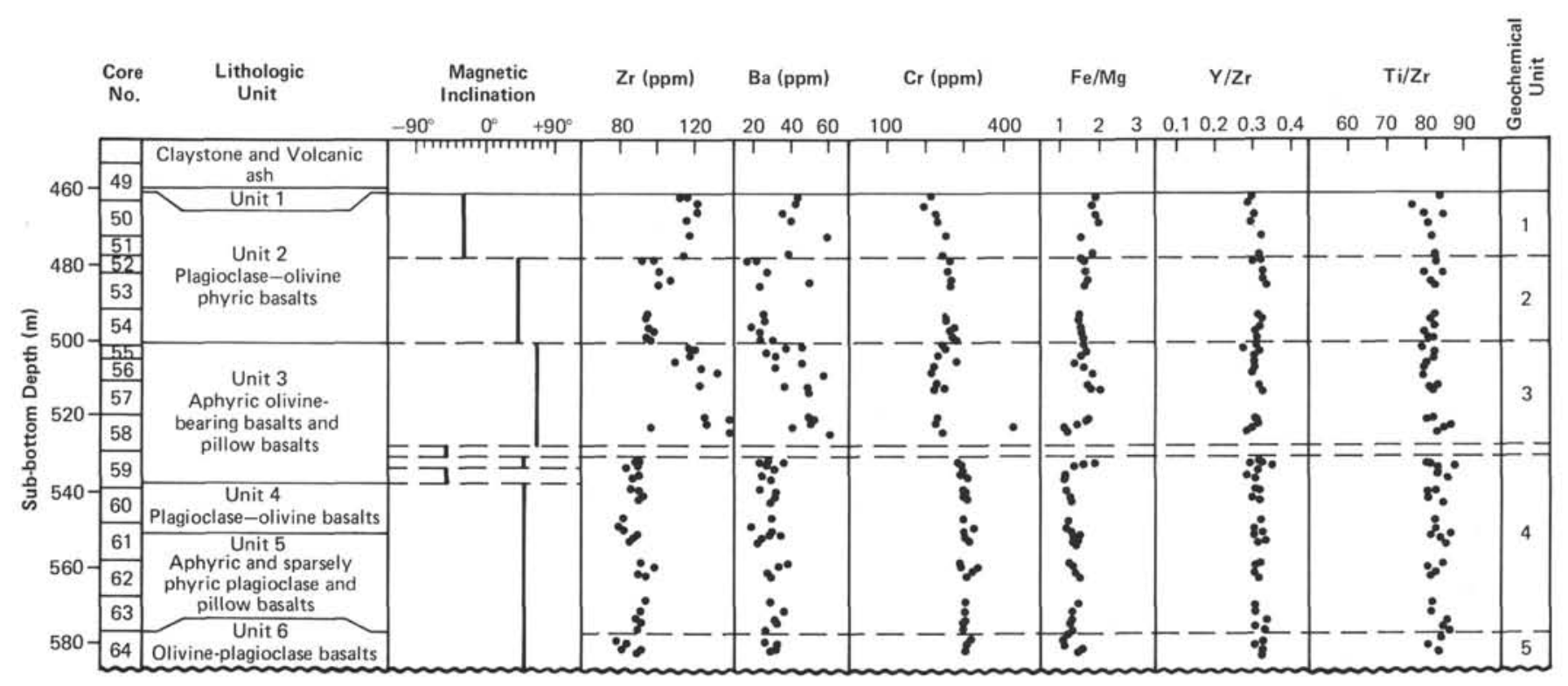

Figure 17. Down-hole variations in lithology, magnetic inclination, and selected geochemical parameters at Site 443. 
TABLE 6

Analyses, Element Ratios, and CIPW Norms for Basalts from Hole $443^{\mathrm{a}}$

\begin{tabular}{|c|c|c|c|c|c|c|c|c|c|c|c|c|}
\hline $\begin{array}{l}\text { Core } \\
\text { Section } \\
\text { Interval }(\mathrm{cm})\end{array}$ & $\begin{array}{r}49 \\
3 \\
127\end{array}$ & $\begin{array}{r}49 \\
4 \\
90\end{array}$ & $\begin{array}{r}50 \\
2 \\
14\end{array}$ & $\begin{array}{r}50 \\
3 \\
80\end{array}$ & $\begin{array}{r}50 \\
4 \\
74\end{array}$ & $\begin{array}{r}51 \\
1 \\
0\end{array}$ & $\begin{array}{r}52 \\
1 \\
2\end{array}$ & $\begin{array}{r}52 \\
1 \\
90\end{array}$ & $\begin{array}{r}52 \\
2 \\
120\end{array}$ & $\begin{array}{r}52 \\
4 \\
16\end{array}$ & $\begin{array}{r}53 \\
2 \\
117\end{array}$ & $\begin{array}{r}53 \\
3 \\
64\end{array}$ \\
\hline $\mathrm{SiO}_{2}(\%)$ & 50.3 & 49.8 & 49.5 & 50.0 & 49.9 & 49.7 & 50.1 & 49.4 & 49.9 & 49.8 & 50.3 & 49.9 \\
\hline $\mathrm{TiO}_{2}$ & 1.55 & 1.56 & 1.56 & 1.60 & 1.56 & 1.58 & 1.58 & 1.32 & 1.36 & 1.35 & 1.47 & 1.40 \\
\hline $\mathrm{Al}_{2} \mathrm{O}_{3}$ & 16.8 & 16.4 & 16.7 & 16.4 & 16.4 & 14.7 & 16.5 & 15.9 & 16.3 & 16.4 & 16.4 & 16.2 \\
\hline $\mathrm{Fe}_{2} \mathrm{O}_{3}$ & 9.29 & 10.58 & 10.41 & 9.64 & 10.84 & 10.16 & 9.13 & 9.57 & 10.17 & 10.00 & 9.43 & 9.93 \\
\hline $\mathrm{MnO}$ & 0.20 & 0.23 & 0.18 & 0.25 & 0.17 & 0.13 & 0.35 & 0.14 & 0.16 & 0.16 & 0.14 & 0.14 \\
\hline $\mathrm{MgO}$ & 5.83 & 6.31 & 6.54 & 5.76 & 6.30 & 7.77 & 5.77 & 7.09 & 7.11 & 7.04 & 6.30 & 6.94 \\
\hline $\mathrm{CaO}$ & 12.37 & 11.78 & 11.56 & 12.73 & 11.58 & 11.42 & 12.79 & 12.43 & 12.11 & 12.04 & 12.23 & 12.01 \\
\hline $\mathrm{Na}_{2} \mathrm{O}$ & 2.91 & 2.68 & 2.69 & 2.78 & 2.79 & 2.65 & 2.86 & 2.76 & 2.65 & 2.70 & 2.90 & 2.80 \\
\hline $\mathrm{K}_{2} \mathrm{O}$ & 0.18 & 0.15 & 0.17 & 0.16 & 0.20 & 0.44 & 0.16 & 0.27 & 0.07 & 0.08 & 0.21 & 0.14 \\
\hline $\mathrm{P}_{2} \mathrm{O}_{5}$ & 0.18 & 0.18 & 0.18 & 0.18 & 0.18 & 0.18 & 0.18 & 0.14 & 0.14 & 0.15 & 0.15 & 0.14 \\
\hline Total & 99.62 & 99.67 & 99.50 & 99.53 & 99.90 & 98.73 & 99.40 & 98.98 & 99.94 & 99.75 & 99.62 & 99.53 \\
\hline $\mathrm{Ni}(\mathrm{ppm})$ & 52 & 49 & 50 & 54 & 56 & 107 & 60 & 99 & 94 & 86 & 107 & 102 \\
\hline $\mathrm{Cr}$ & 219 & 216 & 198 & 224 & 231 & 249 & 244 & 249 & 263 & 256 & 263 & 263 \\
\hline $\mathrm{Zn}$ & 73 & 82 & 79 & 77 & 94 & 76 & 488 & 94 & 482 & 76 & 98 & 131 \\
\hline $\mathrm{Ga}$ & 22 & 22 & 22 & 21 & 19 & 18 & 19 & 19 & 17 & 17 & 19 & 18 \\
\hline $\mathrm{Rb}$ & 2 & 1 & 2 & 1 & 3 & 6 & 2 & 3 & $<1$ & $<1$ & 2 & 1 \\
\hline $\mathrm{Sr}$ & 162 & 155 & 151 & 161 & 152 & 164 & 165 & 168 & 164 & 163 & 171 & 165 \\
\hline $\mathrm{Y}$ & 35 & 34 & 35 & 37 & 35 & 38 & 37 & 30 & 30 & 33 & 35 & 34 \\
\hline $\mathrm{Zr}$ & 116 & 112 & 121 & 120 & 115 & 116 & 114 & 92 & 98 & 101 & 107 & 101 \\
\hline $\mathrm{Nb}$ & 3 & 4 & 5 & 5 & 4 & 7 & 3 & 3 & 1 & 3 & 5 & 4 \\
\hline $\mathrm{Ba}$ & 34 & 43 & 42 & 35 & 40 & 58 & 38 & 22 & 17 & 27 & 49 & 23 \\
\hline $\mathrm{La}$ & 5 & 6 & 7 & 5 & 7 & 7 & 4 & 3 & 2 & 3 & 4 & 5 \\
\hline $\mathrm{Ce}$ & 16 & 14 & 16 & 16 & 16 & 14 & 13 & 13 & 12 & 13 & 15 & 13 \\
\hline $\mathrm{Pb}$ & 1 & 6 & 3 & 7 & 6 & 4 & 6 & 1 & 4 & 5 & 4 & 5 \\
\hline Th & $<1$ & $<1$ & $<1$ & $<1$ & $<1$ & $<1$ & $<1$ & $<1$ & $<1$ & 1 & $<1$ & 2 \\
\hline $\mathrm{Zr} / \mathrm{Nb}$ & 39 & 28 & 24 & 24 & 29 & 17 & 38 & 31 & 98 & 34 & 21 & 25 \\
\hline $\mathrm{Ti} / \mathrm{Zr}$ & 80 & 84 & 77 & 80 & 81 & 82 & 83 & 86 & 83 & 80 & 82 & 83 \\
\hline $\mathrm{Y} / \mathrm{Zr}$ & 0.30 & 0.30 & 0.29 & 0.31 & 0.30 & 0.33 & 0.32 & 0.33 & 0.31 & 0.33 & 0.33 & 0.34 \\
\hline $\mathrm{Ce} / \mathrm{Zr}$ & 0.14 & 0.13 & 0.13 & 0.13 & 0.14 & 0.12 & 0.11 & 0.14 & 0.12 & 0.13 & 0.14 & 0.13 \\
\hline $\mathrm{Ba} / \mathrm{Zr}$ & 0.29 & 0.38 & 0.35 & 0.29 & 0.35 & 0.50 & 0.33 & 0.24 & 0.17 & 0.27 & 0.46 & 0.23 \\
\hline $\mathrm{Ce}_{\mathrm{N}} / \mathrm{Y}_{\mathrm{N}}$ & 1.12 & 1.01 & 1.12 & 1.06 & 1.12 & 0.90 & 0.86 & 1.06 & 0.98 & 0.97 & 1.05 & 0.94 \\
\hline $\mathrm{Fe}_{\text {tot }} / \mathrm{Mg}$ & 1.85 & 1.94 & 1.85 & 1.94 & 2.00 & 1.52 & 1.84 & 1.57 & 1.66 & 1.65 & 1.74 & 1.66 \\
\hline $\mathrm{K} / \mathrm{Rb}$ & 747 & 1253 & 701 & 1303 & 553 & 612 & 668 & 742 & - & - & 880 & 1154 \\
\hline $\mathrm{Ba} / \mathrm{Sr}$ & 0.21 & 0.28 & 0.28 & 0.22 & 0.26 & 0.35 & 0.23 & 0.13 & 0.10 & 0.17 & 0.29 & 0.14 \\
\hline Q & 0.0 & 0.0 & 0.0 & 0.0 & 0. & 0. & 0. & 0.0 & 0. & 0. & 0.0 & 0.0 \\
\hline Or & 1.1 & 0.9 & 1.0 & 0.9 & 1.2 & 2.6 & 1.0 & 1.6 & 0.4 & 0.5 & 1.3 & 0.8 \\
\hline $\mathrm{Ab}$ & 24.7 & 22.8 & 22.9 & 23.6 & 23.6 & 22.7 & 24.3 & 23.6 & 22.4 & 22.9 & 24.6 & 23.8 \\
\hline An & 32.4 & 32.4 & 33.1 & 31.9 & 31.5 & 27.3 & 31.8 & 30.5 & 32.4 & 32.4 & 31.4 & 31.3 \\
\hline $\mathrm{Ne}$ & 0.0 & 0.0 & 0.0 & 0.0 & 0.0 & 0.0 & 0.0 & 0.0 & 0.0 & 0.0 & 0.0 & 0.0 \\
\hline Di & 23.0 & 20.6 & 19.2 & 24.9 & 20.4 & 23.5 & 25.3 & 25.2 & 21.9 & 21.7 & 23.4 & 22.6 \\
\hline $\mathrm{Hy}$ & 11.1 & 15.3 & 14.6 & 11.2 & 14.1 & 12.2 & 9.6 & 5.7 & 13.0 & 12.5 & 10.5 & 10.7 \\
\hline $\mathrm{Ol}$ & 1.9 & 1.9 & 3.1 & 1.4 & 2.9 & 5.4 & 2.1 & 8.0 & 4.3 & 4.5 & 3.3 & 5.1 \\
\hline $\mathrm{Mt}$ & 1.6 & 1.8 & 1.8 & 1.7 & 1.9 & 1.8 & 1.6 & 1.7 & 1.8 & 1.7 & 1.6 & 1.7 \\
\hline II & 3.0 & 3.0 & 3.0 & 3.1 & 3.0 & 3.0 & 3.0 & 2.5 & 2.6 & 2.6 & 2.8 & 2.7 \\
\hline Ap & 0.4 & 0.4 & 0.4 & 0.4 & 0.4 & 0.4 & 0.4 & 0.3 & 0.3 & 0.3 & 0.4 & 0.3 \\
\hline
\end{tabular}

ports the latter explanation, because this section has higher contents of $\mathrm{TiO}_{2}, \mathrm{P}_{2} \mathrm{O}_{5}, \mathrm{Zr}, \mathrm{Ce}$, and $\mathrm{Y}$ than the remainder of lithologic unit 1 .

Lithologic unit 2 consists of plagioclase-phyric basalt (10-15\% plagioclase phenocrysts, composition about $\mathrm{An}_{70}$ ), grading from aphanitic in the chill zone at its upper margin to subophitic at its center. The uppermost 2 meters are amygdaloidal, with 5 to 20 per cent clay- and zeolite-filled vesicles. Relict olivine phenocrysts are seen only in the lower parts of the unit, and rarely exceed 10 per cent of the rock. Augite and magnetite occur throughout the unit, and in the coarsest sections the augite ophitically encloses plagioclase laths. Unit 2 appears to be quite distinct from unit 1 , the former not containing significant ilmenite or apatite and no kaersutite or titaniferous augite.

Major- and trace-element analyses of basalts recovered at Site 444 are given in Table 7. Geochemically, the two lithologic units 1 and 2 are quite distinct; they are therefore treated as two separate geochemical units (Figure 27). The basalts of geochemical unit 1 are nephelinenormative and with a high normative olivine/diopside ratio (Figure 28), which is a function of their low $\mathrm{CaO}$ contents. Geochemical unit 2, however, comprises subalkaline hypersthene- and olivine-normative tholeiites which plot mainly in the plagioclase-tholeiite field on the normative plagioclase-olivine-pyroxene diagram (Figure 29). This is in agreement with the observed phenocryst assemblage (10-15\% plagioclase phenocrysts, $\left.\mathrm{An}_{70}\right)$; the two samples which plot in the olivine-tholeiite field (Core 23-2, $84 \mathrm{~cm}$, and Core 27-5, $68 \mathrm{~cm}$ ) both contain altered groundmass olivine. Core $27-5,68 \mathrm{~cm}$ also contains relict olivine phenocrysts, possibly in part of cumulus origin.

Geochemical unit 444-1 comprises basalts which have low absolute abundances of $\mathrm{SiO}_{2}$ and $\mathrm{CaO}$ compared 
TABLE 6 - Continued

\begin{tabular}{|c|c|c|c|c|c|c|c|c|c|c|c|c|c|}
\hline $\begin{array}{r}54 \\
2\end{array}$ & $\begin{array}{r}54 \\
3\end{array}$ & $\begin{array}{r}54 \\
4\end{array}$ & $\begin{array}{r}54 \\
5\end{array}$ & $\begin{array}{r}54 \\
6\end{array}$ & $\begin{array}{r}54 \\
7\end{array}$ & 55 & 55 & $\begin{array}{r}55 \\
1\end{array}$ & $\begin{array}{r}55 \\
2\end{array}$ & 56 & $\begin{array}{r}56 \\
2\end{array}$ & $\begin{array}{r}56 \\
3\end{array}$ & 57 \\
\hline 44 & 4 & 106 & 31 & 36 & 71 & 81 & 104 & 145 & 115 & 112 & 103 & 115 & 129 \\
\hline 49.5 & 49.6 & 49.5 & 49.6 & 49.6 & 49.7 & 49.7 & 49.1 & 49.4 & 49.3 & 49.6 & 48.7 & 49.9 & 49.5 \\
\hline 1.32 & 1.29 & 1.31 & 1.33 & 1.32 & 1.33 & 1.63 & 1.57 & 1.66 & 1.60 & 1.48 & 1.62 & 1.74 & 1.70 \\
\hline 16.3 & 16.3 & 16.3 & 16.5 & 16.3 & 16.3 & 14.8 & 14.8 & 14.8 & 14.7 & 14.2 & 15.2 & 15.2 & 14.8 \\
\hline 10.09 & 10.05 & 10.18 & 10.08 & 9.89 & 10.03 & 11.20 & 10.71 & 10.98 & 10.64 & 10.51 & 9.46 & 10.47 & 10.61 \\
\hline 0.16 & 0.15 & 0.15 & 0.15 & 0.15 & 0.15 & 0.13 & 0.14 & 0.14 & 0.13 & 0.13 & 0.13 & 0.14 & 0.13 \\
\hline 7.49 & 7.65 & 7.61 & 7.43 & 7.14 & 7.24 & 7.15 & 7.52 & 7.37 & 7.79 & 8.44 & 6.65 & 6.61 & 7.28 \\
\hline 11.86 & 11.74 & 11.76 & 11.78 & 12.07 & 12.16 & 11.38 & 11.87 & 11.49 & 11.60 & 11.22 & 13.88 & 11.41 & 11.67 \\
\hline 2.63 & 2.74 & 2.70 & 2.78 & 2.66 & 2.69 & 2.63 & 2.48 & 2.57 & 2.54 & 2.53 & 2.55 & 2.85 & 2.72 \\
\hline 0.08 & 0.08 & 0.11 & 0.10 & 0.10 & 0.06 & 0.39 & 0.36 & 0.35 & 0.32 & 0.40 & 0.24 & 0.47 & 0.33 \\
\hline 0.14 & 0.15 & 0.14 & 0.14 & 0.13 & 0.13 & 0.17 & 0.17 & 0.17 & 0.17 & 0.17 & 0.18 & 0.19 & 0.18 \\
\hline 99.53 & 99.76 & 99.75 & 99.84 & 99.43 & 99.82 & 99.24 & 98.72 & 98.90 & 98.78 & 98.67 & 98.57 & 99.05 & 98.92 \\
\hline 112 & 117 & 110 & 101 & 103 & 99 & 80 & 111 & 98 & 124 & 156 & 86 & 54 & 103 \\
\hline 254 & 253 & 275 & 262 & 267 & 273 & 244 & 252 & 241 & 234 & 278 & 219 & 213 & 228 \\
\hline 93 & 64 & 107 & 71 & 72 & 112 & 81 & 79 & 81 & 79 & 76 & 75 & 82 & 83 \\
\hline 21 & 19 & 17 & 19 & 19 & 18 & 20 & 18 & 19 & 20 & 16 & 21 & 19 & 23 \\
\hline 1 & $<1$ & 2 & 1 & $<1$ & $<1$ & 5 & 5 & 4 & 3 & 5 & 3 & 14 & 5 \\
\hline 161 & 160 & 158 & 161 & 165 & 161 & 178 & 170 & 168 & 167 & 162 & 176 & 167 & 171 \\
\hline 30 & 31 & 30 & 30 & 30 & 31 & 36 & 33 & 38 & 36 & 34 & 38 & 39 & 39 \\
\hline 95 & 94 & 95 & 98 & 95 & 97 & 118 & 117 & 120 & 116 & 109 & 122 & 131 & 122 \\
\hline 3 & 3 & 3 & 2 & 4 & 2 & 2 & 5 & 7 & 4 & 5 & 3 & 3 & 7 \\
\hline 25 & 26 & 18 & 24 & 24 & 30 & 45 & 37 & 26 & 31 & 45 & 31 & 56 & 36 \\
\hline 4 & 6 & 2 & 4 & 4 & 4 & 7 & 4 & 9 & 4 & 4 & 6 & 5 & 5 \\
\hline 13 & 14 & 12 & 10 & 17 & 12 & 13 & 13 & 19 & 19 & 13 & 18 & 16 & 18 \\
\hline 3 & 4 & 6 & $<1$ & $<1$ & 6 & 5 & 1 & 3 & 3 & 4 & 4 & 2 & 7 \\
\hline 3 & $<1$ & 1 & 1 & $<1$ & $<1$ & 1 & $<1$ & $<1$ & $<1$ & $<1$ & 1 & $<1$ & 1 \\
\hline 32 & 31 & 32 & 49 & 24 & 48 & 59 & 23 & 17 & 29 & 22 & 41 & 44 & 17 \\
\hline 83 & 82 & 83 & 81 & 83 & 82 & 83 & 80 & 83 & 83 & 81 & 80 & 80 & 84 \\
\hline 0.32 & 0.33 & 0.32 & 0.31 & 0.32 & 0.32 & 0.31 & 0.28 & 0.32 & 0.31 & 0.31 & 0.31 & 0.30 & 0.32 \\
\hline 0.14 & 0.15 & 0.13 & 0.10 & 0.18 & 0.12 & 0.11 & 0.11 & 0.16 & 0.16 & 0.12 & 0.15 & 0.12 & 0.15 \\
\hline 0.26 & 0.28 & 0.19 & 0.24 & 0.25 & 0.31 & 0.38 & 0.32 & 0.22 & 0.27 & 0.41 & 0.25 & 0.43 & 0.30 \\
\hline 1.06 & 1.11 & 0.98 & 0.82 & 1.39 & 0.95 & 0.89 & 0.97 & 1.23 & 1.30 & 0.94 & 1.16 & 1.01 & 1.13 \\
\hline 1.56 & 1.52 & 1.55 & 1.57 & 1.61 & 1.61 & 1.82 & 1.65 & 1.73 & 1.58 & 1.44 & 1.65 & 1.84 & 1.69 \\
\hline 664 & - & 440 & 813 & - & - & 642 & 593 & 718 & 880 & 661 & 661 & 279 & 550 \\
\hline 0.16 & 0.16 & 0.11 & 0.15 & 0.15 & 0.19 & 0.25 & 0.22 & 0.15 & 0.19 & 0.28 & 0.18 & 0.34 & 0.21 \\
\hline 0.0 & 0.0 & 0.0 & 0.0 & 0.0 & 0.0 & 0.0 & 0.0 & 0.0 & 0.0 & 0.0 & 0.0 & 0.0 & 0.0 \\
\hline 0.5 & 0.5 & 0.6 & 0.6 & 0.6 & 0.4 & 2.3 & 2.1 & 2.1 & 1.9 & 2.4 & 1.4 & 2.8 & 2.0 \\
\hline 22.4 & 23.2 & 22.9 & 23.6 & 22.6 & 22.8 & 22.4 & 21.3 & 22.0 & 21.8 & 21.7 & 21.9 & 24.3 & 23.3 \\
\hline 32.6 & 32.1 & 32.0 & 32.2 & 32.5 & 32.3 & 27.7 & 28.6 & 28.2 & 28.0 & 26.5 & 29.6 & 27.7 & 27.6 \\
\hline 0.0 & 0.0 & 0.0 & 0.0 & 0.0 & 0.0 & 0.0 & 0.0 & 0.0 & 0.0 & 0.0 & 0.0 & 0.0 & 0.0 \\
\hline 20.9 & 20.7 & 20.9 & 20.8 & 22.0 & 22.3 & 23.0 & 24.5 & 23.2 & 23.8 & 23.4 & 31.9 & 23.1 & 24.3 \\
\hline 12.2 & 10.8 & 10.9 & 10.0 & 12.0 & 11.1 & 14.3 & 11.8 & 13.6 & 13.0 & 14.2 & 2.0 & 11.6 & 10.8 \\
\hline 6.0 & 7.3 & 7.2 & 7.4 & 4.9 & 5.7 & 3.8 & 5.5 & 4.5 & 5.3 & 5.8 & 7.1 & 3.9 & 5.5 \\
\hline 1.8 & 1.8 & 1.8 & 1.8 & 1.7 & 1.7 & 2.0 & 1.9 & 1.9 & 1.9 & 1.9 & 1.7 & 1.8 & 1.9 \\
\hline 2.5 & 2.5 & 2.5 & 2.5 & 2.5 & 2.5 & 3.1 & 3.0 & 3.2 & 3.1 & 2.8 & 3.1 & 3.3 & 3.3 \\
\hline 0.3 & 0.3 & 0.3 & 0.3 & 0.3 & 0.3 & 0.4 & 0.4 & 0.4 & 0.4 & 0.4 & 0.4 & 0.5 & 0.4 \\
\hline
\end{tabular}

with unit 444-2 and the other Shikoku Basin basalts. In addition, the abundances of $\mathrm{Na}_{2} \mathrm{O}, \mathrm{K}_{2} \mathrm{O}, \mathrm{P}_{2} \mathrm{O}_{5}, \mathrm{Rb}, \mathrm{Sr}$, $\mathrm{Nb}, \mathrm{Ba}, \mathrm{La}$, and $\mathrm{Ce}$ are significantly higher than in unit $444-2$, and this has resulted in high $\mathrm{Ba} / \mathrm{Sr}, \mathrm{Ba} / \mathrm{Zr}$, $\mathrm{Ce} / \mathrm{Zr}$, and $\mathrm{Ce}_{\mathrm{N}} / \mathrm{Y}_{\mathrm{N}}$ ratios in the 444-1 basalts. Although both $\mathrm{K}_{2} \mathrm{O}$ and $\mathrm{Rb}$ are high in these basalts (over $1.5 \%$ and $25 \mathrm{ppm}$, respectively), the strong correlation between these two elements, and between them and $\mathrm{Zr}$ (Figure 30), suggests that alteration is not entirely responsible for this enrichment. Indeed, the very high $\mathrm{K} / \mathrm{Rb}$ ratio (generally greater than 1000 ) testifies against low-temperature alteration by sea water, which is known to reduce the $\mathrm{K} / \mathrm{Rb}$ ratio in oceanic basalts (e.g., Hart et al., 1974). The alkaline affinity of the basalts of geochemical unit 444-1 is also supported by the low $\mathrm{Zr} / \mathrm{Nb}$, $\mathrm{Ti} / \mathrm{Zr}$, and $\mathrm{Y} / \mathrm{Zr}$ ratios (Table 7; Figures 31,32 , and
33), and of course by the presence of kaersutite and gest that significant fractionation of these phases has titaniferous augite. In many respects, the basalts of geochemical unit $444 \mathrm{~A}-1$ closely resemble E-type oceanridge basalts from the North Atlantic (Tarney et al., in press).

The high $\mathrm{Ni}$ and $\mathrm{Cr}$ contents of the unit 444-1 basalts indicate that the overall high abundances of many of the HYG elements are not due to high-level fractional crystallization involving pyroxene, olivine, or chrome spinel. However, within-unit variation (Figure 34) does sugoccurred locally, resulting in the low $\mathrm{Ni}$ and $\mathrm{Cr}$ and high HYG-element abundances observed in Core 20-1, 73 $\mathrm{cm}$. This interval is also mineralogically distinct from the remainder of lithologic unit 1 , containing titanaugite, ilmenite, and abundant kaersutite, and it possi- 
TABLE 6 - Continued

\begin{tabular}{|c|c|c|c|c|c|c|c|c|c|c|c|c|}
\hline $\begin{array}{l}\text { Core } \\
\text { Section } \\
\text { Interval }(\mathrm{cm})\end{array}$ & $\begin{array}{r}57 \\
2 \\
8\end{array}$ & $\begin{array}{r}57 \\
2 \\
109\end{array}$ & $\begin{array}{r}58 \\
1 \\
58\end{array}$ & $\begin{array}{r}58 \\
1 \\
94\end{array}$ & $\begin{array}{r}58 \\
2 \\
99\end{array}$ & $\begin{array}{r}58 \\
3 \\
29\end{array}$ & $\begin{array}{r}58 \\
4 \\
30\end{array}$ & $\begin{array}{r}59 \\
1 \\
71\end{array}$ & $\begin{array}{r}59 \\
1 \\
129\end{array}$ & $\begin{array}{r}59 \\
2 \\
15\end{array}$ & $\begin{array}{r}59 \\
2 \\
91\end{array}$ & $\begin{array}{r}59 \\
3 \\
4\end{array}$ \\
\hline $\mathrm{SiO}_{2}(\%)$ & 49.9 & 49.4 & 49.7 & 50.0 & 50.3 & 46.1 & 50.4 & 49.5 & 49.7 & 49.7 & 50.0 & 49.7 \\
\hline $\mathrm{TiO}_{2}$ & 1.69 & 1.71 & 1.68 & 1.91 & 1.83 & 1.36 & 1.93 & 1.28 & 1.19 & 1.25 & 1.24 & 1.22 \\
\hline $\mathrm{Al}_{2} \mathrm{O}_{3}$ & 15.2 & 14.8 & 15.3 & 15.4 & 15.5 & 11.1 & 14.9 & 16.0 & 16.3 & 16.2 & 16.4 & 16.3 \\
\hline $\mathrm{Fe}_{2} \mathrm{O}_{3}$ & 10.36 & 11.34 & 10.28 & 9.84 & 9.42 & 13.85 & 9.72 & 9.95 & 9.55 & 9.06 & 8.94 & 9.13 \\
\hline $\mathrm{MnO}$ & 0.14 & 0.18 & 0.14 & 0.14 & 0.10 & 0.17 & 0.10 & 0.17 & 0.17 & 0.13 & 0.13 & 0.13 \\
\hline $\mathrm{MgO}$ & 6.73 & 6.46 & 6.91 & 6.90 & 7.52 & 14.01 & 9.33 & 6.08 & 6.84 & 7.84 & 7.34 & 7.95 \\
\hline $\mathrm{CaO}$ & 12.20 & 12.28 & 11.07 & 11.03 & 10.69 & 7.48 & 9.26 & 12.94 & 12.09 & 11.58 & 12.07 & 11.62 \\
\hline $\mathrm{Na}_{2} \mathrm{O}$ & 2.68 & 2.51 & 2.86 & 2.96 & 3.09 & 1.73 & 2.85 & 2.71 & 2.75 & 2.68 & 2.72 & 2.71 \\
\hline $\mathrm{K}_{2} \mathrm{O}$ & 0.39 & 0.47 & 0.41 & 0.49 & 0.22 & 0.19 & 0.26 & 0.29 & 0.31 & 0.10 & 0.06 & 0.10 \\
\hline $\mathrm{P}_{2} \mathrm{O}_{5}$ & 0.17 & 0.17 & 0.18 & 0.20 & 0.18 & 0.16 & 0.20 & 0.13 & 0.12 & 0.12 & 0.12 & 0.11 \\
\hline Total & 99.39 & 99.33 & 98.56 & 98.93 & 98.83 & 96.13 & 99.00 & 99.06 & 99.00 & 98.66 & 99.02 & 98.99 \\
\hline $\mathrm{Ni}(\mathrm{ppm})$ & 92 & 90 & 79 & 62 & 73 & 405 & 115 & 92 & 100 & 104 & 108 & 113 \\
\hline $\mathrm{Cr}_{\mathrm{r}}$ & 237 & 222 & 233 & 227 & 225 & 428 & 246 & 286 & 285 & 291 & 294 & 297 \\
\hline $\mathrm{Zn}$ & 98 & 94 & 89 & 80 & 84 & 102 & 86 & 68 & 64 & 87 & 78 & 79 \\
\hline $\mathrm{Ga}$ & 22 & 18 & 19 & 21 & 22 & 12 & 21 & 19 & 16 & 18 & 19 & 17 \\
\hline $\mathrm{Rb}$ & 10 & 6 & 17 & 15 & 3 & 4 & 3 & 6 & 7 & 1 & $<1$ & 1 \\
\hline $\mathrm{Sr}$ & 166 & 159 & 168 & 175 & 178 & 97 & 154 & 173 & 165 & 149 & 156 & 154 \\
\hline$Y$ & 40 & 41 & 39 & 44 & 40 & 29 & 40 & 29 & 29 & 27 & 29 & 28 \\
\hline $\mathrm{Zr}$ & 124 & 123 & 124 & 138 & 126 & 96 & 138 & 90 & 88 & 91 & 84 & 87 \\
\hline $\mathrm{Nb}$ & 8 & 5 & 5 & 5 & 7 & 7 & 7 & 2 & 3 & 3 & 2 & 3 \\
\hline $\mathrm{Ba}$ & 48 & 49 & 49 & 52 & 50 & 40 & 60 & 28 & 36 & 23 & 28 & 31 \\
\hline $\mathrm{La}$ & 6 & 6 & 5 & 6 & 7 & 6 & 5 & 3 & 3 & 4 & 3 & 4 \\
\hline $\mathrm{Ce}$ & 16 & 11 & 16 & 17 & 17 & 14 & 18 & 12 & 8 & 11 & 11 & 8 \\
\hline $\mathrm{Pb}$ & 7 & 2 & 1 & 4 & 5 & 5 & 5 & 2 & 4 & 5 & 6 & 4 \\
\hline Th & $<1$ & 1 & $<1$ & $<1$ & 2 & $<1$ & $<1$ & $<1$ & $<1$ & 1 & $<1$ & $<1$ \\
\hline $\mathrm{Zr} / \mathrm{Nb}$ & 15 & 25 & 25 & 28 & 18 & 14 & 20 & 45 & 29 & 30 & 42 & 29 \\
\hline $\mathrm{Ti} / \mathrm{Zr}_{\mathrm{r}}$ & 82 & 83 & 81 & 83 & 87 & 85 & 84 & 85 & 81 & 82 & 88 & 84 \\
\hline $\mathrm{Y} / \mathrm{Zr}$ & 0.32 & 0.33 & 0.31 & 0.32 & 0.32 & 0.30 & 0.29 & 0.32 & 0.33 & 0.30 & 0.35 & 0.32 \\
\hline $\mathrm{Ce} / \mathrm{Zr}$ & 0.13 & 0.09 & 0.13 & 0.12 & 0.13 & 0.15 & 0.13 & 0.13 & 0.09 & 0.12 & 0.13 & 0.09 \\
\hline $\mathrm{Ba} / \mathrm{Zr}$ & 0.39 & 0.40 & 0.40 & 0.38 & 0.40 & 0.42 & 0.43 & 0.31 & 0.41 & 0.25 & 0.33 & 0.36 \\
\hline $\mathrm{Ce}_{\mathrm{N}} / \mathrm{Y}_{\mathrm{N}}$ & 0.98 & 0.66 & 1.01 & 0.95 & 1.04 & 1.19 & 1.11 & 1.02 & 0.68 & 1.00 & 0.93 & 0.70 \\
\hline $\mathrm{Fe}_{\text {tot }} / \mathrm{Mg}$ & 1.79 & 2.04 & 1.73 & 1.65 & 1.45 & 1.15 & 1.21 & 1.90 & 1.62 & 1.34 & 1.41 & 1.33 \\
\hline $\mathrm{K} / \mathrm{Rb}$ & 321 & 656 & 202 & 270 & 595 & 396 & 706 & 396 & 374 & 863 & - & 855 \\
\hline $\mathrm{Ba} / \mathrm{Sr}$ & 0.29 & 0.31 & 0.29 & 0.30 & 0.28 & 0.41 & 0.39 & 0.16 & 0.22 & 0.15 & 0.18 & 0.20 \\
\hline Q & 0.0 & 0.0 & 0.0 & 0.0 & 0.0 & 0.0 & 0.0 & 0. & 0.0 & 0.0 & 0.0 & 0.0 \\
\hline Or & 2.3 & 2.8 & 2.5 & 2.9 & 1.3 & 1.2 & 1.5 & 1.7 & 1.9 & 0.6 & 0.3 & 0.6 \\
\hline $\mathrm{Ab}$ & 22.8 & 21.4 & 24.6 & 25.3 & 26.5 & 15.2 & 24.4 & 23.1 & 23.5 & 23.0 & 23.2 & 23.2 \\
\hline An & 28.3 & 27.8 & 28.0 & 27.7 & 28.2 & 22.8 & 27.4 & 30.9 & 31.5 & 32.4 & 32.7 & 32.4 \\
\hline $\mathrm{Ne}$ & 0.0 & 0.0 & 0.0 & 0.0 & 0.0 & 0.0 & 0.0 & 0.0 & 0.0 & 0.0 & 0.0 & 0.0 \\
\hline $\mathrm{Di}$ & 25.7 & 26.6 & 21.7 & 21.4 & 19.7 & 12.0 & 14.3 & 27.1 & 23.1 & 20.4 & 22.0 & 20.4 \\
\hline Hy & 10.7 & 10.9 & 12.6 & 11.6 & 14.0 & 26.2 & 22.6 & 6.1 & 8.4 & 13.5 & 12.9 & 12.2 \\
\hline 01 & 3.8 & 3.8 & 4.2 & 4.2 & 3.9 & 15.8 & 3.1 & 5.6 & 6.5 & 5.1 & 3.8 & 6.2 \\
\hline Mt & 1.8 & 2.0 & 1.8 & 1.7 & 1.7 & 2.5 & 1.7 & 1.7 & 1.7 & 1.6 & 1.6 & 1.6 \\
\hline 11 & 3.2 & 3.3 & 3.2 & 3.7 & 3.5 & 2.7 & 3.7 & 2.5 & 2.3 & 2.4 & 2.4 & 2.3 \\
\hline Ap & 0.4 & 0.4 & 0.4 & 0.5 & 0.4 & 0.4 & 0.5 & 0.3 & 0.3 & 0.3 & 0.3 & 0.3 \\
\hline
\end{tabular}

bly results from within-sill fractionation. The HYG-element ratios of Core $20-1,73 \mathrm{~cm}$, however, are indistinguishable from the rest of geochemical unit 444-1.

Geochemical unit 444-2 comprises basalts which are chemically similar to those recovered at Sites 442 and 443. Thus, they contain neither normative nepheline nor normative quartz (Figure 28). They have low abundances of $\mathrm{K}_{2} \mathrm{O}, \mathrm{P}_{2} \mathrm{O}_{5}, \mathrm{Rb}, \mathrm{Sr}$, and $\mathrm{Ba}$, and have $\mathrm{Ce} / \mathrm{Zr}$, $\mathrm{Ba} / \mathrm{Zr}, \mathrm{Ce} / \mathrm{Y}$, and $\mathrm{K} / \mathrm{Rb}$ ratios similar to those of the other Shikoku Basin basalts. Nevertheless, they have slightly higher $\mathrm{Nb}(5-9 \mathrm{ppm})$, and consequently they have lower $\mathrm{Zr} / \mathrm{Nb}$ ratios than the basalts from either Site 442 or Site 443 , and they have significantly lower $\mathrm{Ti} / \mathrm{Zr}$ ratios (Table 7; Figures 31 and 32 ). $\mathrm{Cr}$ and $\mathrm{Ni}$ are less than $100 \mathrm{ppm}$ and $280 \mathrm{ppm}$, respectively, and the moderately high $\mathrm{Fe} / \mathrm{Mg}$ ratios (greater than 1.5) testify to the fractionated nature of these samples. The higher $\mathrm{Ni}$ and $\mathrm{Cr}$ in Core $27-5,68 \mathrm{~cm}$, at the base of lithologic unit 2 may be due to the presence of relict olivine phenocrysts, possibly in part of cumulus origin. Within-unit fractionation of $\mathrm{Ni}$ is clearly indicated by Figure 34 .

A plot of Ce against Y (Figure 35) shows that the basalts of geochemical unit 444-1 are light-REE enriched, $\mathrm{Ce}_{\mathrm{N}} / \mathrm{Y}_{\mathrm{N}}$ ranging from 2.6 to 2.8 , and $\mathrm{Ce}_{\mathrm{N}}$ over 30. Geochemical unit $444-2$ is also enriched in light $\mathrm{REE}$, but to a much lesser degree $\left(\mathrm{Ce}_{\mathrm{N}} / \mathrm{Y}_{\mathrm{N}} 1.12-1.46\right)$, and thus more closely resembles the basalts from Sites 442 and 443 (Figure 35).

The available data suggest that it is unlikely that geochemical units 444-1 and 444-2 were derived from the same parental melt by differing degrees of fractional crystallization. $\mathrm{Ni}$ and $\mathrm{Cr}$ are highest in 444-1 (apart from 20-1, $73 \mathrm{~cm}$ ), which also contains basalts most enriched in HYG elements. It is also unlikely that the magmas of the two units could have been derived from a compositionally identical source by varying the nature 
TABLE 6 - Continued

\begin{tabular}{|c|c|c|c|c|c|c|c|c|c|c|c|c|c|}
\hline $\begin{array}{r}59 \\
3 \\
101\end{array}$ & $\begin{array}{r}59 \\
4 \\
138\end{array}$ & $\begin{array}{r}59 \\
5 \\
65\end{array}$ & $\begin{array}{r}60 \\
1 \\
21\end{array}$ & $\begin{array}{r}60 \\
1 \\
80\end{array}$ & $\begin{array}{r}60 \\
2 \\
106\end{array}$ & $\begin{array}{r}60 \\
4 \\
0\end{array}$ & $\begin{array}{r}60 \\
6 \\
104\end{array}$ & $\begin{array}{r}61 \\
1 \\
93\end{array}$ & $\begin{array}{r}61 \\
2 \\
107\end{array}$ & $\begin{array}{r}61 \\
3 \\
13\end{array}$ & $\begin{array}{r}61 \\
3 \\
29\end{array}$ & $\begin{array}{r}61 \\
3 \\
101\end{array}$ & $\begin{array}{r}61 \\
4 \\
69\end{array}$ \\
\hline 49.7 & 49.1 & 49.6 & 48.8 & 49.3 & 49.1 & 49.3 & 48.6 & 48.7 & 48.9 & 49.4 & 49.7 & 49.4 & 49.7 \\
\hline 1.27 & 1.26 & 1.25 & 1.18 & 1.23 & 1.24 & 1.27 & 1.14 & 1.11 & 1.20 & 1.21 & 1.23 & 1.23 & 1.22 \\
\hline 16.0 & 15.9 & 16.3 & 15.8 & 15.8 & 15.8 & 15.8 & 15.9 & 15.9 & 16.0 & 16.1 & 16.1 & 16.1 & 16.2 \\
\hline 9.28 & 8.58 & 8.30 & 9.11 & 9.39 & 9.64 & 9.51 & 9.15 & 9.39 & 9.50 & 9.39 & 9.35 & 9.36 & 9.49 \\
\hline 0.13 & 0.12 & 0.13 & 0.15 & 0.16 & 0.16 & 0.17 & 0.16 & 0.15 & 0.15 & 0.15 & 0.13 & 0.13 & 0.14 \\
\hline 7.66 & 8.71 & 8.54 & 9.16 & 9.11 & 8.57 & 8.56 & 9.04 & 9.56 & 8.24 & 7.07 & 7.17 & 8.11 & 7.78 \\
\hline 11.70 & 11.79 & 11.52 & 10.90 & 10.67 & 11.07 & 11.35 & 11.16 & 11.06 & 11.86 & 13.06 & 12.34 & 11.88 & 12.07 \\
\hline 2.77 & 2.68 & 2.69 & 2.61 & 2.69 & 2.52 & 2.56 & 2.57 & 2.47 & 2.53 & 2.59 & 2.60 & 2.52 & 2.62 \\
\hline 0.23 & 0.07 & 0.28 & 0.06 & 0.09 & 0.09 & 0.07 & 0.17 & 0.07 & 0.10 & 0.21 & 0.21 & 0.05 & 0.05 \\
\hline 0.11 & 0.12 & 0.11 & 0.12 & 0.11 & 0.12 & 0.12 & 0.11 & 0.11 & 0.11 & 0.11 & 0.10 & 0.11 & 0.11 \\
\hline 98.80 & 98.26 & 98.75 & 97.94 & 98.52 & 98.38 & 98.64 & 97.95 & 98.58 & 98.52 & 99.32 & 98.98 & 98.85 & 99.39 \\
\hline 145 & 124 & 140 & 122 & 124 & 103 & 96 & 147 & 183 & 127 & 108 & 117 & 105 & 117 \\
\hline 301 & 291 & 311 & 293 & 303 & 299 & 305 & 299 & 324 & 299 & 302 & 304 & 308 & 315 \\
\hline 75 & 61 & 80 & 57 & 57 & 70 & 66 & 74 & 61 & 70 & 74 & 68 & 102 & 73 \\
\hline 19 & 20 & 19 & 18 & 18 & 18 & 19 & 14 & 17 & 19 & 19 & 19 & 18 & 17 \\
\hline 3 & 1 & 4 & $<1$ & $<1$ & 1 & 2 & 3 & 2 & 3 & 5 & 5 & 1 & 1 \\
\hline 150 & 200 & 151 & 142 & 144 & 137 & 147 & 172 & 140 & 199 & 167 & 167 & 151 & 152 \\
\hline 29 & 26 & 27 & 26 & 29 & 28 & 29 & 26 & 25 & 27 & 28 & 27 & 29 & 27 \\
\hline 91 & 90 & 87 & 85 & 91 & 92 & 90 & 82 & 80 & 83 & 89 & 88 & 86 & 85 \\
\hline 4 & 1 & 2 & 1 & 3 & 1 & 3 & 1 & $<1$ & 2 & $<1$ & 4 & 2 & 3 \\
\hline 31 & 24 & 29 & 23 & 31 & 31 & 33 & 29 & 19 & 29 & 34 & 28 & 24 & 21 \\
\hline 3 & 3 & 2 & 2 & 2 & 3 & 3 & 2 & 5 & 3 & 2 & 4 & 2 & 3 \\
\hline 10 & 11 & 10 & 13 & 8 & 10 & 6 & 12 & 11 & 10 & 12 & 9 & 13 & 16 \\
\hline 5 & 2 & 4 & 2 & $<1$ & 4 & 6 & 3 & 6 & 5 & 4 & 3 & $<1$ & 4 \\
\hline$<1$ & $<1$ & $<1$ & 1 & $<1$ & 3 & $<1$ & $<1$ & 4 & 1 & $<1$ & 5 & $<1$ & $<1$ \\
\hline 23 & 90 & 43 & 85 & 30 & 92 & 30 & 82 & - & 42 & - & 22 & 43 & 28 \\
\hline 84 & 84 & 86 & 83 & 81 & 81 & 85 & 83 & 83 & 87 & 82 & 84 & 86 & 86 \\
\hline 0.32 & 0.29 & 0.31 & 0.31 & 0.32 & 0.30 & 0.32 & 0.32 & 0.31 & 0.33 & 0.31 & 0.31 & 0.34 & 0.32 \\
\hline 0.11 & 0.12 & 0.11 & 0.15 & 0.09 & 0.11 & 0.07 & 0.15 & 0.14 & 0.12 & 0.13 & 0.10 & 0.15 & 0.19 \\
\hline 0.34 & 0.27 & 0.33 & 0.27 & 0.34 & 0.34 & 0.37 & 0.35 & 0.24 & 0.35 & 0.38 & 0.32 & 0.28 & 0.25 \\
\hline 0.85 & 1.04 & 0.91 & 1.23 & 0.68 & 0.88 & 0.51 & 1.13 & 1.08 & 0.91 & 1.05 & 0.82 & 1.10 & 1.46 \\
\hline 1.40 & 1.14 & 1.13 & 1.15 & 1.20 & 1.30 & 1.29 & 1.17 & 1.14 & 1.34 & 1.54 & 1.51 & 1.34 & 1.41 \\
\hline 628 & 573 & 585 & - & - & 706 & 311 & 465 & 278 & 279 & 345 & 344 & 448 & 407 \\
\hline 0.21 & 0.12 & 0.19 & 0.16 & 0.22 & 0.23 & 0.22 & 0.17 & 0.14 & 0.15 & 0.20 & 0.17 & 0.16 & 0.14 \\
\hline 0.0 & 0.0 & 0.0 & 0.0 & 0.0 & 0.0 & 0.0 & 0.0 & 0.0 & 0.0 & 0.0 & 0.0 & 0.0 & 0.0 \\
\hline 1.4 & 0.4 & 1.7 & 0.4 & 0.5 & 0.5 & 0.4 & 1.0 & 0.4 & 0.6 & 1.2 & 1.2 & 0.3 & 0.3 \\
\hline 23.7 & 23.1 & 23.0 & 22.5 & 23.1 & 21.7 & 22.0 & 22.2 & 21.2 & 21.7 & 22.1 & 22.2 & 21.6 & 22.3 \\
\hline 30.9 & 31.6 & 32.0 & 31.9 & 31.3 & 32.2 & 31.9 & 32.1 & 32.6 & 32.4 & 31.9 & 32.0 & 32.7 & 32.6 \\
\hline 0.0 & 0.0 & 0.0 & 0.0 & 0.0 & 0.0 & 0.0 & 0.0 & 0.0 & 0.0 & 0.0 & 0.0 & 0.0 & 0.0 \\
\hline 22.0 & 21.9 & 20.3 & 18.2 & 17.6 & 18.5 & 20.1 & 19.5 & 18.1 & 21.6 & 26.6 & 23.8 & 21.3 & 21.9 \\
\hline 9.8 & 8.9 & 9.8 & 12.8 & 13.8 & 15.6 & 13.5 & 9.4 & 12.6 & 10.3 & 6.0 & 10.9 & 13.5 & 11.9 \\
\hline 7.0 & 9.1 & 8.3 & 9.1 & 8.6 & 6.2 & 7.0 & 11.0 & 10.2 & 8.2 & 7.2 & 4.8 & 5.5 & 5.9 \\
\hline 1.6 & 1.5 & 1.5 & 1.6 & 1.7 & 1.7 & 1.7 & 1.6 & 1.7 & 1.7 & 1.6 & 1.6 & 1.6 & 1.7 \\
\hline 2.4 & 2.4 & 2.4 & 2.3 & 2.4 & 2.4 & 2.4 & 2.2 & 2.1 & 2.3 & 2.3 & 2.4 & 2.4 & 2.3 \\
\hline 0.3 & 0.3 & 0.3 & 0.3 & 0.3 & 0.3 & 0.2 & 0.1 & 0.3 & 0.3 & 0.3 & 0.3 & 0.3 & 0.3 \\
\hline
\end{tabular}

and degree of partial melting, because the low bulk-distribution coefficients of $\mathrm{K}, \mathrm{Rb}, \mathrm{Zr}, \mathrm{La}, \mathrm{Ce}$, and $\mathrm{Nb}$ (see, for example Wood et al., 1979) make it difficult to change ratios of the more-HYG elements, even if very small, but still realistic, degrees of partial melting are invoked. Instead, we suggest that geochemical units 444-1 and 444-2 were derived from compositionally distinct mantle sources, the source of 444-1 being enriched in $\mathrm{Ce}, \mathrm{La}, \mathrm{Nb}, \mathrm{K}, \mathrm{Rb}, \mathrm{Ba}$, and $\mathrm{Sr}$ relative to the source of 444-2.

Both units 444-1 and 444-2 were emplaced as sills long after basement formation; therefore, it is not surprising that the basalts of unit 444-1 are compositionally similar to E-type ocean-ridge basalts, which are characteristic of off-axis volcanic activity (see, for example Tarney et al., 1979, in press; Wood et al., 1979). Even unit 444-2, which is not as enriched as 444-1, is more enriched than $\mathrm{N}$-type ocean-ridge basalts, having higher
$\mathrm{Ce} / \mathrm{Y}$ and lower $\mathrm{Ti} / \mathrm{Zr}$ and $\mathrm{Zr} / \mathrm{Nb}$ ratios; the $444-2$ basalts more closely resemble $\mathrm{T}$-type ocean-ridge basalts. The Th, Hf, and La data presented by Wood et al. (this volume) also support this comparison.

\section{NORTHEAST PHILIPPINE BASIN}

Two sites are in the northeast corner of the Philippine Basin. Site 445 is in a small basin on the southern side of the Daito Ridge (Figure 1), and the only igneous rocks recovered at this site are a few clasts and pebbles in some of the conglomerates. These have been studied in detail by Mills (this volume).

Site 446 is in the Daito Basin, south of the Daito Ridge (Figure 1), at $24^{\circ} 42.04^{\prime} \mathrm{N}, 132^{\circ} 46.49^{\prime} \mathrm{E}$, in 4952 meters of water. Two holes were drilled, and basalts interbedded with sediments were encountered beneath about 380 meters of sediment; the oldest sediment gave an age of early Eocene. From several lines of evidence, 
TABLE 6 - Continued

\begin{tabular}{|c|c|c|c|c|c|c|c|c|c|c|c|c|c|c|}
\hline $\begin{array}{l}\text { Core } \\
\text { Section } \\
\text { Interval (cm) }\end{array}$ & $\begin{array}{r}62 \\
1 \\
94\end{array}$ & $\begin{array}{r}62 \\
2 \\
27\end{array}$ & $\begin{array}{r}62 \\
3 \\
56\end{array}$ & $\begin{array}{r}62 \\
4 \\
18\end{array}$ & $\begin{array}{r}63 \\
1 \\
140\end{array}$ & $\begin{array}{r}63 \\
3 \\
116\end{array}$ & $\begin{array}{r}63 \\
5 \\
5\end{array}$ & $\begin{array}{r}63 \\
6 \\
12\end{array}$ & $\begin{array}{r}63 \\
8 \\
54\end{array}$ & $\begin{array}{r}64 \\
1 \\
78\end{array}$ & $\begin{array}{r}64 \\
2 \\
97\end{array}$ & $\begin{array}{r}64 \\
3 \\
0\end{array}$ & $\begin{array}{r}64 \\
4 \\
5\end{array}$ & $\begin{array}{r}64 \\
4 \\
32\end{array}$ \\
\hline $\mathrm{SiO}_{2}(\%)$ & 50.0 & 49.9 & 49.9 & 50.1 & 50.0 & 49.8 & 49.7 & 49.5 & 50.0 & 48.7 & 48.4 & 48.7 & 49.8 & 49.8 \\
\hline $\mathrm{TiO}_{2}$ & 1.29 & 1.32 & 1.25 & 1.29 & 1.27 & 1.24 & 1.27 & 1.29 & 1.30 & 1.15 & 1.11 & 1.14 & 1.27 & 1.24 \\
\hline $\mathrm{Al}_{2} \mathrm{O}_{3}$ & 15.9 & 15.9 & 15.9 & 16.0 & 15.9 & 15.9 & 15.9 & 15.8 & 16.0 & 15.8 & 15.6 & 15.7 & 15.9 & 15.9 \\
\hline $\mathrm{Fe}_{2} \mathrm{O}_{3}$ & 8.58 & 8.63 & 9.28 & 8.99 & 9.25 & 8.61 & 9.47 & 9.45 & 9.44 & 9.59 & 9.47 & 9.45 & 9.66 & 9.50 \\
\hline $\mathrm{MnO}$ & 0.12 & 0.12 & 0.15 & 0.13 & 0.13 & 0.11 & 0.15 & 0.16 & 0.15 & 0.15 & 0.15 & 0.15 & 0.15 & 0.14 \\
\hline $\mathrm{MgO}$ & 8.03 & 7.58 & 7.66 & 6.82 & 7.10 & 7.45 & 8.39 & 8.52 & 8.11 & 9.47 & 10.09 & 9.82 & 7.03 & 7.38 \\
\hline $\mathrm{CaO}$ & 11.95 & 11.69 & 12.32 & 12.62 & 12.27 & 12.59 & 11.32 & 11.37 & 11.53 & 10.67 & 10.65 & 10.71 & 12.40 & 12.29 \\
\hline $\mathrm{Na}_{2} \mathrm{O}$ & 2.55 & 2.83 & 2.56 & 2.62 & 2.73 & 2.68 & 2.67 & 2.65 & 2.81 & 2.38 & 2.23 & 2.31 & 2.60 & 2.63 \\
\hline $\mathrm{K}_{2} \mathrm{O}$ & 0.07 & 0.25 & 0.25 & 0.25 & 0.26 & 0.23 & 0.10 & 0.09 & 0.09 & 0.11 & 0.11 & 0.13 & 0.26 & 0.24 \\
\hline $\mathrm{P}_{2} \mathrm{O}_{5}$ & 0.13 & 0.13 & 0.11 & 0.12 & 0.12 & 0.12 & 0.12 & 0.13 & 0.14 & 0.12 & 0.12 & 0.12 & 0.12 & 0.12 \\
\hline Total & 98.61 & 98.38 & 99.41 & 98.93 & 99.00 & 98.72 & 99.10 & 99.02 & 99.56 & 98.17 & 97.94 & 98.20 & 99.14 & 99.31 \\
\hline $\mathrm{Ni}$ (ppm) & 94 & 97 & 127 & 120 & 116 & 180 & 94 & 97 & 96 & 139 & 170 & 144 & 96 & 119 \\
\hline $\mathrm{Cr}$ & 290 & 293 & 321 & 310 & 306 & 307 & 308 & 300 & 300 & 319 & 323 & 311 & 310 & 304 \\
\hline $\mathrm{Zn}$ & 67 & 69 & 75 & 99 & 77 & 64 & 86 & 77 & 63 & 55 & 62 & 63 & 76 & 75 \\
\hline $\mathrm{Ga}$ & 19 & 18 & 19 & 19 & 20 & 17 & 18 & 19 & 21 & 20 & 17 & 17 & 16 & 17 \\
\hline $\mathrm{Rb}$ & 1 & 3 & 4 & 5 & 5 & 3 & 1 & 1 & 2 & 2 & 2 & 3 & 6 & 3 \\
\hline $\mathrm{Sr}$ & 158 & 161 & 163 & 165 & 165 & 166 & 146 & 152 & 148 & 137 & 137 & 136 & 173 & 193 \\
\hline $\mathrm{Y}$ & 29 & 30 & 28 & 30 & 29 & 28 & 30 & 28 & 30 & 27 & 26 & 26 & 30 & 29 \\
\hline $\mathrm{Zr}$ & 91 & 98 & 90 & 94 & 93 & 91 & 89 & 91 & 90 & 82 & 78 & 84 & 91 & 89 \\
\hline $\mathrm{Nb}$ & $<1$ & 2 & 2 & 1 & 1 & 2 & 2 & 2 & 3 & 2 & 1 & 1 & 3 & 4 \\
\hline $\mathrm{Ba}$ & 37 & 33 & 28 & 29 & 28 & 35 & 32 & 33 & 26 & 30 & 25 & 33 & 32 & 28 \\
\hline $\mathrm{La}$ & 3 & 2 & 3 & 4 & 6 & 2 & 3 & 2 & 4 & 4 & 2 & 5 & 4 & 2 \\
\hline $\mathrm{Ce}$ & 11 & 8 & 13 & 11 & 16 & 9 & 9 & 10 & 14 & 12 & 11 & 11 & 11 & 9 \\
\hline $\mathrm{Pb}$ & 3 & 2 & 5 & 6 & 6 & 1 & 5 & 7 & 2 & 6 & 5 & 5 & 4 & 6 \\
\hline Th & $<1$ & $<1$ & 1 & $<1$ & $<1$ & $<1$ & $<1$ & $<1$ & 2 & 1 & 1 & $<1$ & $<1$ & $<1$ \\
\hline $\mathrm{Zr} / \mathrm{Nb}$ & - & 49 & 45 & 94 & 93 & 45 & 45 & 45 & 30 & 41 & 78 & 84 & 30 & 22 \\
\hline $\mathrm{Ti} / \mathrm{Zr}_{\mathrm{r}}$ & 85 & 81 & 83 & 82 & 82 & 82 & 86 & 85 & 87 & 84 & 85 & 81 & 84 & 84 \\
\hline $\mathrm{Y} / \mathrm{Zr}$ & 0.32 & 0.31 & 0.31 & 0.32 & 0.31 & 0.31 & 0.34 & 0.31 & 0.33 & 0.33 & 0.33 & 0.31 & 0.33 & 0.33 \\
\hline $\mathrm{Ce} / \mathrm{Zr}$ & 0.12 & 0.08 & 0.14 & 0.12 & 0.17 & 0.10 & 0.10 & 0.11 & 0.16 & 0.15 & 0.14 & 0.13 & 0.12 & 0.10 \\
\hline $\mathrm{Ba} / \mathrm{Zr}$ & 0.41 & 0.34 & 0.31 & 0.31 & 0.30 & 0.38 & 0.36 & 0.36 & 0.29 & 0.37 & 0.32 & 0.39 & 0.35 & 0.31 \\
\hline$C e_{N} / Y_{N}$ & 0.93 & 0.65 & 1.14 & 0.90 & 1.36 & 0.79 & 0.74 & 0.88 & 1.15 & 1.09 & 1.04 & 1.04 & 0.90 & 0.76 \\
\hline $\mathrm{Fe}_{\text {tot }} / \mathrm{Mg}$ & 1.24 & 1.32 & 1.40 & 1.53 & 1.51 & 1.34 & 1.31 & 1.29 & 1.35 & 1.17 & 1.09 & 1.12 & 1.59 & 1.49 \\
\hline $\mathrm{K} / \mathrm{Rb}$ & 606 & 695 & 513 & 408 & 423 & 642 & 797 & 764 & 369 & 465 & 452 & 357 & 362 & 672 \\
\hline $\mathrm{Ba} / \mathrm{Sr}$ & 0.23 & 0.20 & 0.17 & 0.18 & 0.17 & 0.21 & 0.22 & 0.22 & 0.18 & 0.22 & 0.18 & 0.24 & 0.18 & 0.15 \\
\hline Q & 0.0 & 0.0 & 0. & 0. & 0. & 0. & 0. & 0. & 0. & 0. & 0. & 0. & 0. & 0.0 \\
\hline Or & 0.4 & 1.5 & 1.5 & 1.5 & 1.5 & 1.4 & 0.6 & 0.5 & 0.5 & 0.7 & 0.7 & 0.8 & 1.6 & 1.4 \\
\hline $\mathrm{Ab}$ & 21.9 & 24.3 & 21.8 & 22.4 & 23.3 & 23.0 & 22.8 & 22.6 & 23.9 & 20.5 & 19.3 & 19.9 & 22.2 & 22.4 \\
\hline An & 32.3 & 30.5 & 31.4 & 31.4 & 30.7 & 31.1 & 31.4 & 31.4 & 31.0 & 32.7 & 32.9 & 32.6 & 31.2 & 31.2 \\
\hline $\mathrm{Ne}$ & 0.0 & 0.0 & 0.0 & 0.0 & 0.0 & 0.0 & 0.0 & 0.0 & 0.0 & 0.0 & 0.0 & 0.0 & 0.0 & 0.0 \\
\hline $\mathrm{Di}$ & 21.9 & 22.4 & 23.9 & 25.3 & 24.5 & 25.5 & 19.8 & 20.1 & 20.8 & $16.6^{\circ}$ & 16.4 & 16.8 & 24.6 & 24.0 \\
\hline Hy & 15.7 & 10.6 & 10.9 & 11.5 & 9.8 & 8.1 & 13.7 & 13.0 & 11.5 & 16.4 & 16.9 & 16.5 & 10.4 & 10.0 \\
\hline $\mathrm{Ol}$ & 2.8 & 5.5 & 5.5 & 2.7 & 5.1 & 6.0 & 6.4 & 7.1 & 7.0 & 8.1 & 8.9 & 8.4 & 4.8 & 5.8 \\
\hline Mt & 1.5 & 1.5 & 1.6 & 1.6 & 1.6 & 1.5 & 1.7 & 1.7 & 1.6 & 1.7 & 1.7 & 1.7 & 1.7 & 1.7 \\
\hline Il & 2.5 & 2.5 & 2.4 & 2.5 & 2.4 & 2.4 & 2.4 & 2.5 & 2.5 & 2.2 & 2.2 & 2.2 & 2.4 & 2.4 \\
\hline Ap & 0.3 & 0.3 & 0.3 & 0.3 & 0.3 & 0.3 & 0.3 & 0.3 & 0.3 & 0.3 & 0.3 & 0.3 & 0.3 & 0.3 \\
\hline
\end{tabular}

aNormative values based on $\mathrm{Fe}_{2} \mathrm{O}_{3} / \mathrm{FeO}$ ratio of $0.15 . \mathrm{Fe}_{2} \mathrm{O}_{3}$ is total iron as $\mathrm{Fe}_{2} \mathrm{O}_{3}$. Ce $/ \mathrm{Y}_{\mathrm{N}}$ represents ratio of chondrite-normalized values.

but principally the abundance of thermally altered sediments above and below most of the igneous units, all the basalts at Site 446 are considered to have been emplaced as sills (Site 446 report, this volume) during post-early Eocene time. Basement, which was not reached, is thought to have an early Eocene age at this site.

The basaltic rocks recovered from Hole 446A have been subdivided into 9 lithologic and 15 geochemical units (Figure 36), and 23 individual sills have been recognized. These vary in thickness from 0.3 to 23 meters and can be delineated by aphanitic or occasionally even glassy chill zones. Two main lithologic types of basalt and dolerite have been identified: a kaersutite, hornblende-bearing variety (lithologic units 1, 4, 5), and a hornblende-free variety (lithologic units $2,3,6,7,8,9$ ).

Lithologic units 1,4 , and 5 contain 5 to 15 per cent of primary kaersutite, which occurs either as free-growing anhedral laths, as overgrowths on clinopyroxene, or as fibrous clumps. The pyroxene in these kaersutite-bearing samples has a distinct purplish-pink coloration indicative of a high $\mathrm{TiO}_{2}$ content, and the rocks contain abundant granular magnetite and skeletal ilmenite. Plagioclase and (in unit 5) olivine are the other main mineral components; apatite crystals were observed in lithologic units 4 and 5 .

Lithologic unit 1 comprises two equigranular, highly vesicular (up to $30 \%$ vesicles), fine- to medium-grained basaltic sills. The sills have an intergranular texture, and appear to be fresh. Lithologic unit 4 also comprises two petrographically similar sills, separated by a claystone interbed. The thicker, upper sill has been divided into two sub-units by a break in recovery; the upper sub-unit (4A) consists of highly vesicular $(25-40 \%)$, aphanitic to coarse-grained basalt. Sub-unit $4 \mathrm{~B}$ is a fine-grained, amygdaloidal basalt, and sub-unit $4 \mathrm{C}$ is a smaller-scale version of the upper sill. Unit 4 is cut by several clay- 


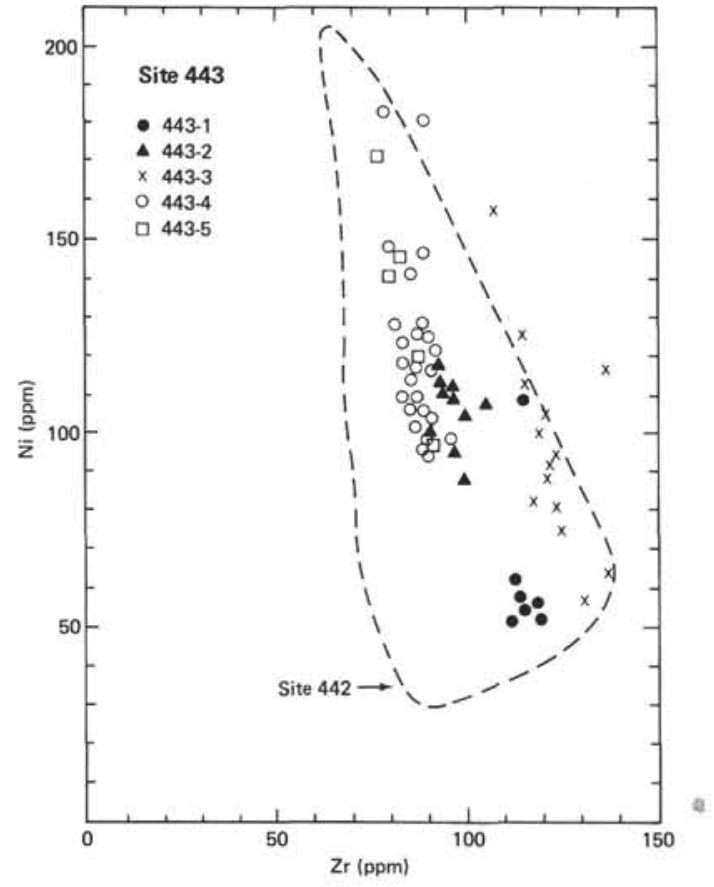

Figure 18. Ni versus $\mathrm{Zr}$, Site 443. The dashed line outlines the field of Site 442 basalts.

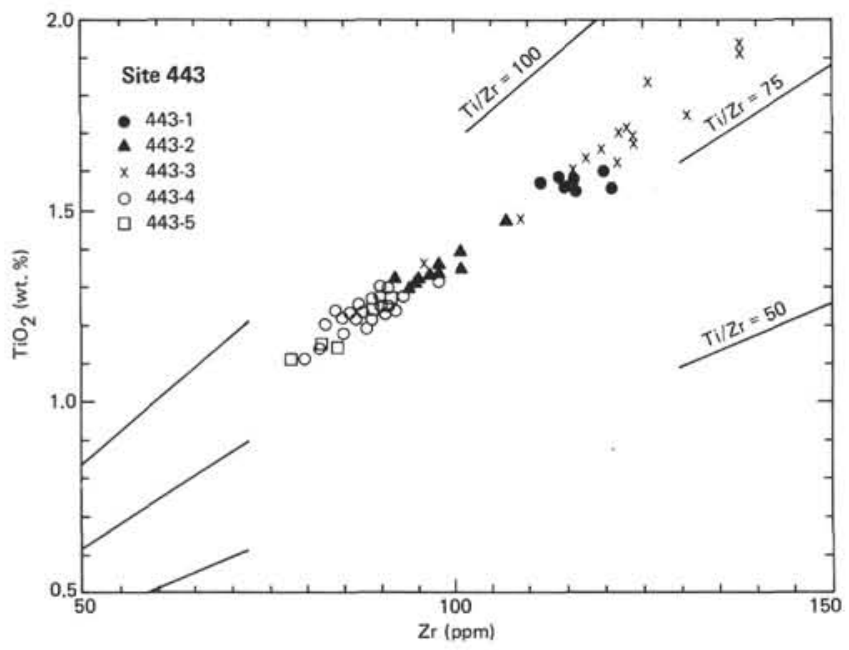

Figure 19. $\mathrm{TiO}_{2}$ versus $\mathrm{Zr}$, Site 443. Note the strong colinearity of the data points.

and carbonate-lined veins or fractures, and the vesicles in the lower sections of both sills are filled by light-olivegreen clay and calcite. Lithologic unit 5 is a single sill, containing up to 35 per cent dark, chloritic material, which is occasionally recognizable as pseudomorphs after olivine (0.1-1.0 mm across).

The kaersutite-free units essentially comprise plagioclase, augite, titanomagnetite, and chloritic or clayey pseudomorphs after olivine. Lithologic unit 2 is a single aphyric-basalt sill, the upper part of which is vesicular; the basalt is fine- to medium-grained, with intersertal texture. The unit is cut by several veins lined with clay, quartz, and pyrite. Lithologic unit 3 comprises three

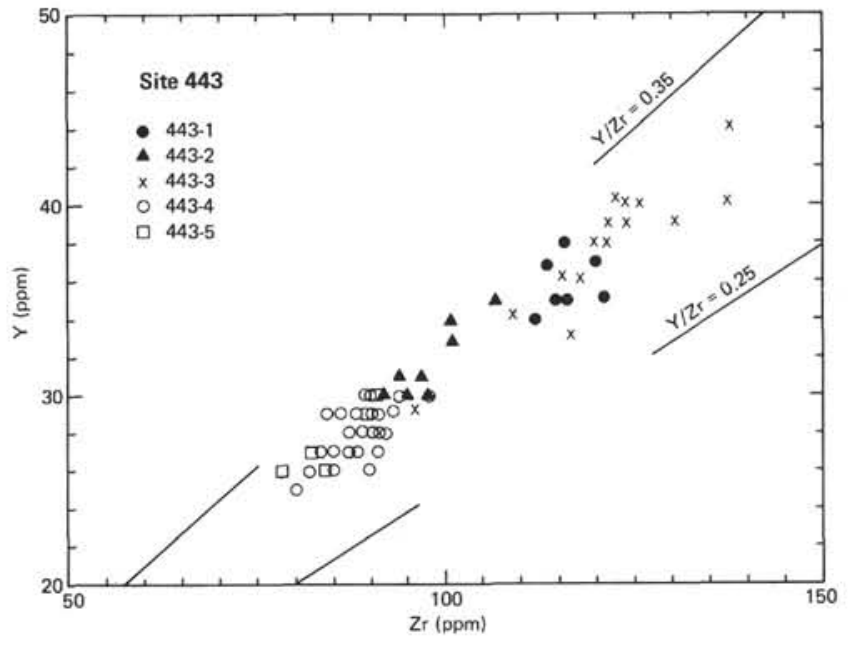

Figure 20. $Y$ versus $Z r$, Site 443.

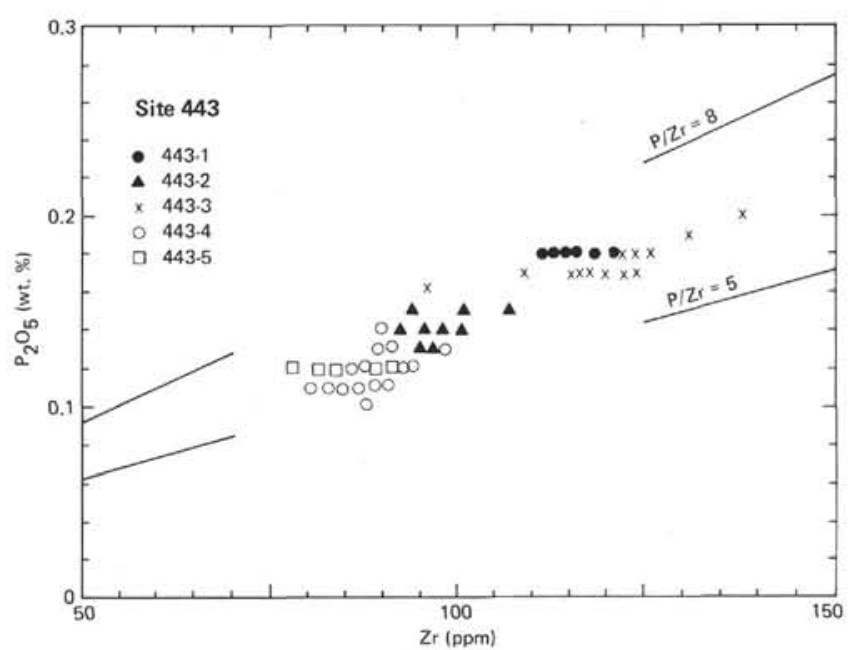

Figure 21. $\mathrm{P}_{2} \mathrm{O}_{5}$ versus $\mathrm{Zr}$, Site 443. The colinearity of the data points should be compared with the scatter observed in the data from Site 442 (Figures 10 and 15).

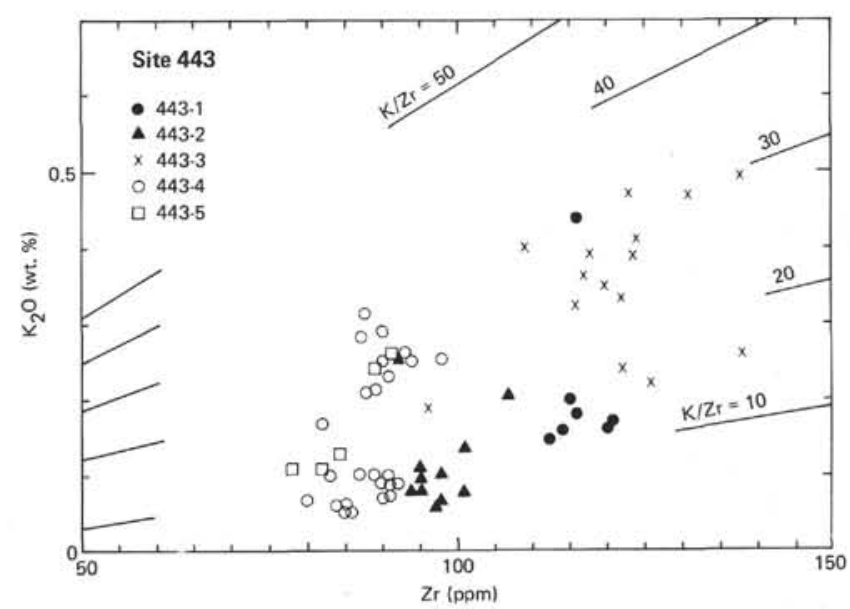

Figure 22. $\mathrm{K}_{2} \mathrm{O}$ versus $\mathrm{Zr}$, Site 443 . 


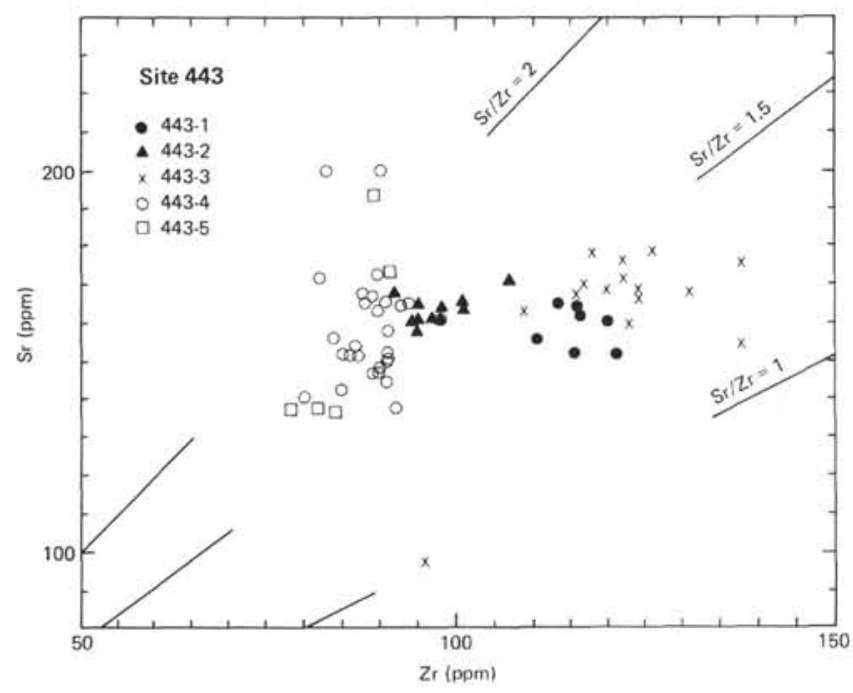

Figure 23. Sr versus $\mathrm{Zr}$, Site 443.

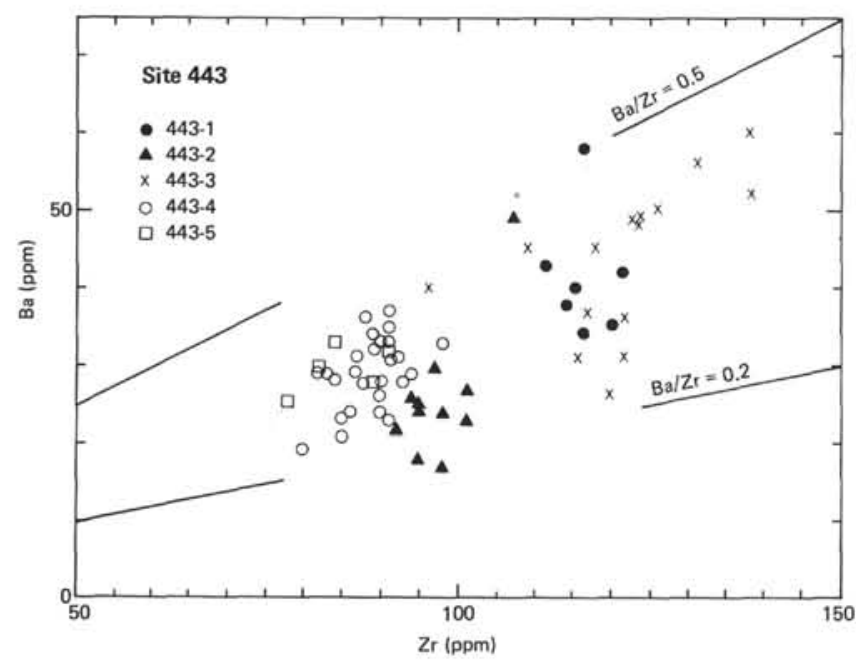

Figure 24. Ba versus $\mathrm{Zr}$, Site 443.

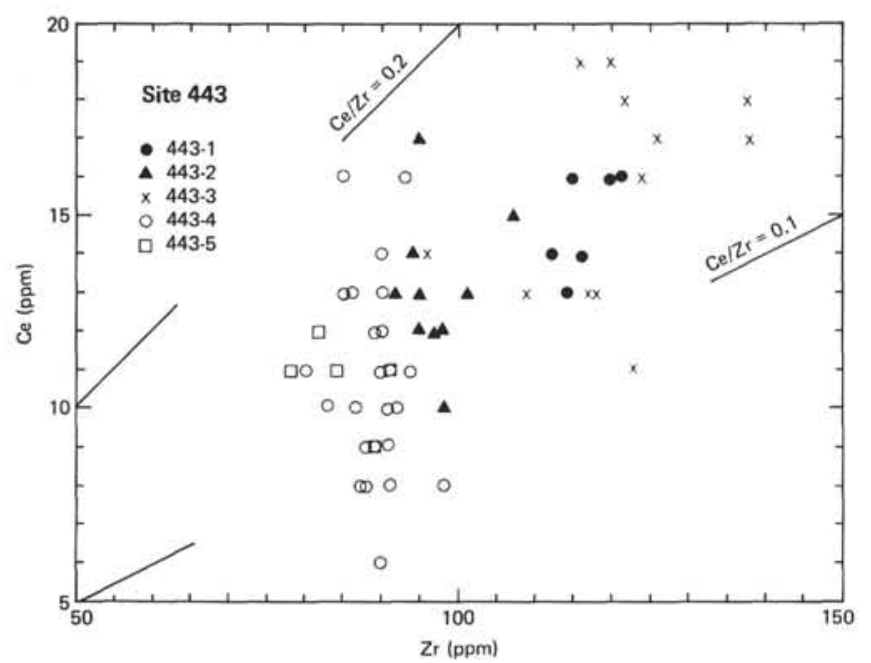

Figure 25. Ce versus $\mathrm{Zr}$, Site 443.

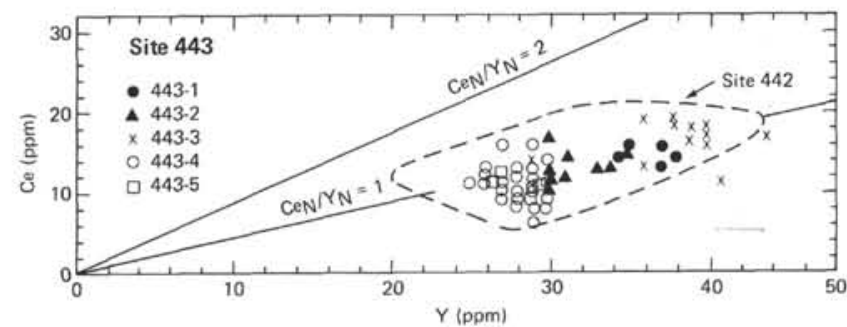

Figure 26. Ce versus $Y$, Site 443. Field for Site 442 shown for comparison.

sills: sub-unit $3 \mathrm{~A}$ is a plagioclase $(40 \%)$ and augite $(1 \%)$ microphyric basalt; $3 \mathrm{~B}$ is a plagioclase $(3-15 \%)$ and augite $(0.8 \%)$ phyric basalt; and $3 \mathrm{C}$ is a sparsely plagioclase-pyroxene phyric basalt. All sub-units contain a few clay pseudomorphs after olivine, and $3 \mathrm{~B}$ and $3 \mathrm{C}$ have vesicular upper portions.

Lithologic unit 6 comprises six fine-grained, sparsely phyric basalt sills. The basalt is essentially non-vesicular, apart from the top of unit $6 \mathrm{C}$, which contains 10 to 15 per cent vesicles, and rare plagioclase phenocrysts. Lithologic unit 7, however, comprises two almost identical plagioclase-pyroxene-phyric sills separated by a claystone interbed. The upper sill, sub-unit 7A, has a glassy margin which contains a few red-brown pseudomorphs, possibly after olivine. Lithologic units 8 and 9 both comprise aphyric basalt sills, the former containing rare plagioclase and augite microphenocrysts. Both units are cut by clay- and calcite-lined fractures.

Major- and trace-element data for the basalts recovered at Site 446 are listed in Tables 8 and 9. The nine lithologic units comprise at least 15 geochemical units (Figure 36), although lithologic units 6B and 6C were not sampled. It is apparent that breaks between chemical units usually coincide with breaks between lithological units or sub-units, but where a significant change in chemistry occurs without a change in petrography the units are divided into geochemical sub-units.

Perhaps the most noticeable feature of the Daito Basin samples, apart from their high contents of $\mathrm{TiO}_{2}$, total iron, $\mathrm{K}_{2} \mathrm{O}, \mathrm{P}_{2} \mathrm{O}_{5}, \mathrm{Sr}, \mathrm{Ba}, \mathrm{La}, \mathrm{Ce}, \mathrm{Zr}$, and $\mathrm{Nb}$ compared with the basalts from the Shikoku Basin, is their wide range in chemical composition, both within and among the various units. This is illustrated by the downhole plot (Figure 36), and also by the normative basalt diagram of Figure 37, where the range of chemistry-from nepheline-normative basalts to quartz-normative tholeiites-becomes apparent. Two interdigitated groups have been recognized: an alkalic-basalt suite comprising geochemical units 446-1, 4, 5, 7a, and 10a; and a tholeiitic-basalt suite comprising units 446-2, $3,6,7 \mathrm{~b}, 8,9,10 \mathrm{~b}, 11,12,13,14$, and 15 . This division is not based solely on the presence or absence of normative nepheline; indeed, most of the samples in unit 446-5 (and all those in 446-7a) contain normative hypersthene. Rather, the alkalic basalts are characterized by low normative-hypersthene contents, or the presence of normative nepheline, and also by high abundances of cer- 


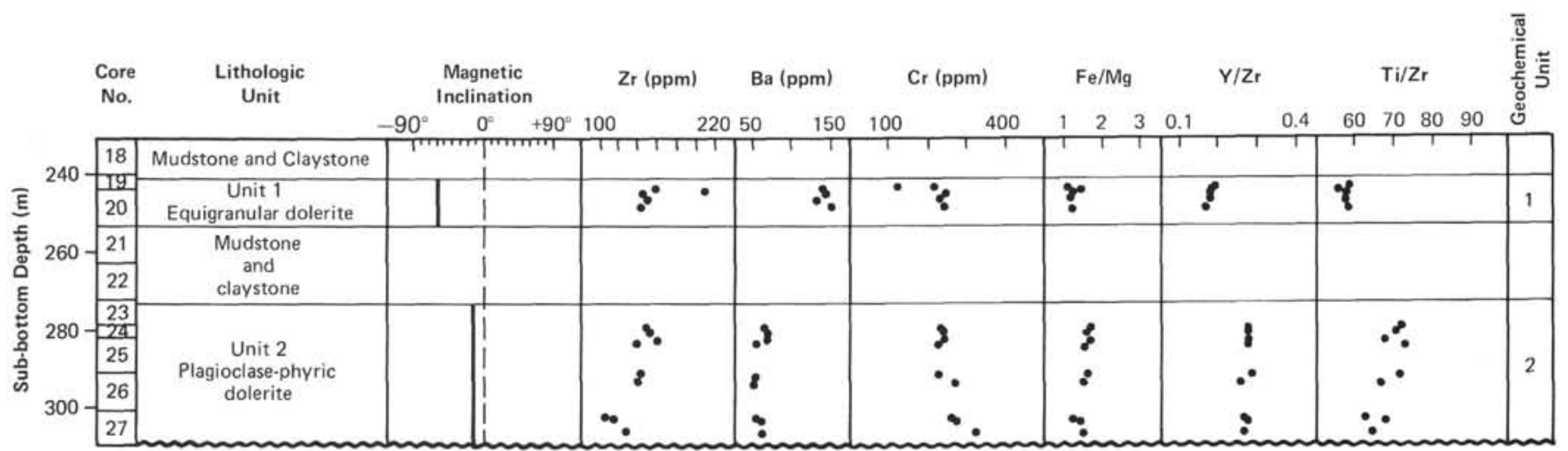

Figure 27. Down-hole variations in lithology, magnetic inclination, and selected geochemical parameters, Site 444.

TABLE 7

Analyses, Element Ratios, and CIPW Norms for Basalts from Hole $444 \mathrm{~A}^{\mathrm{a}}$

\begin{tabular}{|c|c|c|c|c|c|c|c|c|c|c|c|c|c|c|c|}
\hline $\begin{array}{l}\text { Core } \\
\text { Section } \\
\text { Interval (cm) }\end{array}$ & $\begin{array}{r}20 \\
1 \\
22\end{array}$ & $\begin{array}{r}20 \\
1 \\
73\end{array}$ & $\begin{array}{r}20 \\
2 \\
0\end{array}$ & $\begin{array}{r}20 \\
3 \\
39\end{array}$ & $\begin{array}{r}20 \\
4 \\
37\end{array}$ & $\begin{array}{r}23 \\
2 \\
84\end{array}$ & $\begin{array}{r}24 \\
1 \\
87\end{array}$ & $\begin{array}{r}24 \\
2 \\
73\end{array}$ & $\begin{array}{r}25 \\
1 \\
118\end{array}$ & $\begin{array}{r}25 \\
3 \\
50\end{array}$ & $\begin{array}{r}26 \\
1 \\
49\end{array}$ & $\begin{array}{r}26 \\
3 \\
0\end{array}$ & $\begin{array}{r}27 \\
2 \\
62\end{array}$ & $\begin{array}{r}27 \\
3 \\
21\end{array}$ & $\begin{array}{r}27 \\
5 \\
68\end{array}$ \\
\hline $\mathrm{SiO}_{2}(\%)$ & 47,7 & 50.3 & 47.2 & 47.1 & 46.7 & 49.6 & 49.7 & 50.0 & 50.2 & 49.8 & 50.0 & 50.0 & 48.9 & 49.7 & 49.1 \\
\hline $\mathrm{TiO}_{2}^{2}$ & 1.55 & 1.94 & 1.41 & 1.43 & 1.43 & 1.50 & 1.57 & 1.59 & 1.61 & 1.46 & 1.47 & 1.37 & 1.15 & 1.33 & 1.38 \\
\hline $\mathrm{Al}_{2} \mathrm{O}_{3}$ & 16.0 & 16.2 & 16.4 & 16.2 & 16.5 & 15.9 & 15.9 & 16.0 & 16.1 & 16.7 & 16.4 & 17.1 & 16.4 & 16.6 & 15.6 \\
\hline $\mathrm{Fe}_{2} \mathrm{O}_{3}$ & 8.65 & 7.96 & 8.49 & 8.45 & 8.36 & 9.69 & 10.03 & 10.32 & 10.15 & 9.50 & 9.62 & 8.96 & 8.91 & 9.60 & 10.02 \\
\hline $\mathrm{MnO}$ & 0.15 & 0.15 & 0.16 & 0.15 & 0.15 & 0.31 & 0.16 & 0.17 & 0.17 & 0.16 & 0.17 & 0.15 & 0.19 & 0.17 & 0.17 \\
\hline $\mathrm{MgO}$ & 8.67 & 6.25 & 7.70 & 7.89 & 7.69 & 7.01 & 6.89 & 7.25 & 6.87 & 6.87 & 6.72 & 6.54 & 8.09 & 7.35 & 7.46 \\
\hline $\mathrm{CaO}$ & 8.07 & 8.59 & 8.43 & 8.77 & 8.10 & 12.41 & 11.27 & 11.09 & 11.36 & 11.61 & 11.86 & 12.22 & 10.96 & 11.73 & 12.67 \\
\hline $\mathrm{Na}_{2} \mathrm{O}$ & 3.05 & 3.86 & 3.62 & 3.56 & 3.92 & 2.72 & 2.71 & 2.72 & 2.82 & 2.64 & 2.67 & 2.75 & 2.69 & 2.61 & 2.49 \\
\hline $\mathrm{K}_{2} \mathrm{O}$ & 1.78 & 2.31 & 1.90 & 1.80 & 2.06 & 0.24 & 0.17 & 0.22 & 0.16 & 0.17 & 0.24 & 0.19 & 0.34 & 0.23 & 0.22 \\
\hline $\mathrm{P}_{2} \mathrm{O}_{5}$ & 0.31 & 0.39 & 0.34 & 0.29 & 0.26 & 0.20 & 0.21 & 0.22 & 0.23 & 0.21 & 0.21 & 0.20 & 0.18 & 0.19 & 0.20 \\
\hline Total & 95.95 & 97.92 & 95.68 & 95.71 & 95.18 & 99.63 & 98.63 & 99.56 & 99.73 & 99.14 & 99.40 & 99.49 & 97.87 & 99.48 & 99.29 \\
\hline $\mathrm{Ni}(\mathrm{ppm})$ & 137 & 26 & 180 & 172 & 182 & 71 & 59 & 59 & 53 & 57 & 62 & 69 & 97 & 95 & 114 \\
\hline $\mathrm{Cr}$ & 222 & 127 & 246 & 234 & 245 & 253 & 238 & 245 & 245 & 235 & 234 & 273 & 262 & 277 & 325 \\
\hline $\mathrm{Zn}$ & 67 & 79 & 91 & 62 & 57 & 76 & 70 & 73 & 63 & 62 & 88 & 83 & 62 & 72 & 85 \\
\hline $\mathrm{Ga}$ & 20 & 18 & 20 & 19 & 18 & 19 & 20 & 24 & 20 & 20 & 21 & 19 & 22 & 17 & 19 \\
\hline $\mathrm{Rb}$ & 14 & 16 & 17 & 14 & 16 & 3 & 2 & 3 & 2 & 2 & 3 & 2 & 2 & 2 & 2 \\
\hline $\mathrm{Sr}$ & 336 & 391 & 295 & 284 & 280 & 163 & 160 & 153 & 160 & 159 & 159 & 167 & 266 & 159 & 380 \\
\hline Y & 30 & 38 & 27 & 27 & 25 & 35 & 37 & 37 & 40 & 33 & 36 & 32 & 29 & 33 & 34 \\
\hline $\mathrm{Zr}_{\mathrm{r}}$ & 158 & 209 & 146 & 149 & 145 & 118 & 130 & 134 & 141 & 120 & 123 & 123 & 109 & 117 & 128 \\
\hline $\mathrm{Nb}$ & 24 & 31 & 24 & 23 & 24 & 5 & 8 & 9 & 7 & 8 & 8 & 6 & 6 & 6 & 7 \\
\hline $\mathrm{Ba}$ & 141 & 177 & 146 & 135 & 151 & 57 & 65 & 70 & 71 & 57 & 54 & 52 & 54 & 63 & 62 \\
\hline $\mathrm{La}$ & 14 & 18 & 15 & 14 & 11 & 6 & 8 & 8 & 8 & 6 & 8 & 5 & 7 & 6 & 6 \\
\hline $\mathrm{Ce}$ & 33 & 42 & 30 & 31 & 26 & 17 & 22 & 21 & 20 & 17 & 19 & 17 & 15 & 15 & 16 \\
\hline $\mathrm{Pb}$ & 5 & 4 & 4 & 4 & 5 & 4 & 3 & 5 & 3 & 6 & 5 & 3 & 8 & $<1$ & 4 \\
\hline $\mathrm{Th}$ & 3 & 4 & 3 & $<1$ & 2 & 1 & $<1$ & 1 & 2 & 1 & 2 & 1 & $<1$ & $<1$ & $<1$ \\
\hline $\mathrm{Zr} / \mathrm{Nb}$ & 7 & 7 & 6 & 6 & 6 & 24 & 16 & 15 & 20 & 15 & 15 & 20 & 18 & 19 & 18 \\
\hline $\mathrm{Ti} / \mathrm{Zr}$ & 59 & 56 & 58 & 58 & 59 & 76 & 72 & 71 & 68 & 73 & 72 & 67 & 63 & 68 & 65 \\
\hline $\mathrm{Y} / \mathrm{Zr}$ & 0.19 & 0.18 & 0.18 & 0.18 & 0.17 & 0.30 & 0.28 & 0.28 & 0.28 & 0.28 & 0.29 & 0.26 & 0.27 & 0.28 & 0.27 \\
\hline $\mathrm{Ce} / \mathrm{Zr}$ & 0.21 & 0.20 & 0.21 & 0.21 & 0.18 & 0.14 & 0.17 & 0.16 & 0.14 & 0.14 & 0.15 & 0.14 & 0.14 & 0.13 & 0.13 \\
\hline $\mathrm{Ba} / \mathrm{Zr}$ & 0.89 & 0.85 & 1.00 & 0.91 & 1.04 & 0.48 & 0.50 & 0.52 & 0.50 & 0.47 & 0.44 & 0.42 & 0.50 & 0.54 & 0.48 \\
\hline $\mathrm{Ce}_{\mathrm{N}} / \mathrm{Y}_{\mathrm{N}}$ & 2.70 & 2.71 & 2.73 & 2.82 & 2.55 & 1.19 & 1.46 & 1.39 & 1.23 & 1.27 & 1.30 & 1.30 & 1.27 & 1.12 & 1.16 \\
\hline $\mathrm{Fe}_{\text {tot }} / \mathrm{Mg}$ & 1.16 & 1.48 & 1.28 & 1.24 & 1.26 & 1.60 & 1.69 & 1.65 & 1.71 & 1.60 & 1.66 & 1.59 & 1.28 & 1.51 & 1.56 \\
\hline $\mathrm{K} / \mathrm{Rb}$ & 1055 & 1198 & 928 & 1067 & 1069 & 664 & 693 & 606 & 664 & 693 & 659 & 797 & 1403 & 959 & 892 \\
\hline $\mathrm{Ba} / \mathrm{Sr}$ & 0.42 & 0.45 & 0.49 & 0.48 & 0.54 & 0.35 & 0.41 & 0.46 & 0.44 & 0.36 & 0.34 & 0.31 & 0.20 & 0.40 & 0.16 \\
\hline Q & 0.0 & 0.0 & 0.0 & 0.0 & 0.0 & 0.0 & 0.0 & 0.0 & 0.0 & 0.0 & 0.0 & 0.0 & 0.0 & 0.0 & 0.0 \\
\hline Or & 11.0 & 13.9 & 11.7 & 11.1 & 12.8 & 1.4 & 1.0 & 1.3 & 0.9 & 1.0 & 1.4 & 1.1 & 2.0 & 1.4 & 1.3 \\
\hline $\mathrm{Ab}$ & 25.7 & 27.4 & 22.1 & 21.3 & 19.7 & 23.1 & 23.2 & 23.1 & 23.9 & 22.5 & 22.7 & 23.4 & 23.3 & 22.2 & 21.2 \\
\hline An & 25.8 & 20.5 & 23.9 & 24.0 & 22.3 & 30.6 & 31.3 & 30.9 & 30.9 & 33.6 & 32.4 & 33.8 & 32.5 & 32.9 & 30.9 \\
\hline $\mathrm{Ne}$ & 0.6 & 3.2 & 5.4 & 5.5 & 8.2 & 0.0 & 0.0 & 0.0 & 0.0 & 0.0 & 0.0 & 0.0 & 0.0 & 0.0 & 0.0 \\
\hline $\mathrm{Di}$ & 11.3 & 16.7 & 14.3 & 15.8 & 14.7 & 24.4 & 19.6 & 18.7 & 19.7 & 18.9 & 20.8 & 21.1 & 17.8 & 19.8 & 25.4 \\
\hline $\mathrm{Hy}$ & 0.0 & 0.0 & 0.0 & 0.0 & 0.0 & 8.4 & 17.0 & 17.0 & 16.3 & 16.4 & 14.7 & 12.1 & 10.6 & 13.5 & 8.5 \\
\hline OI & 19.5 & 11.5 & 16.7 & 16.4 & 16.5 & 6.1 & 1.6 & 2.7 & 1.9 & 1.8 & 2.2 & 3.0 & 8.8 & 4.7 & 6.9 \\
\hline $\mathrm{Mt}$ & 1.6 & 1.4 & 1.5 & 1.5 & 1.5 & 1.7 & 1.8 & 1.8 & 1.8 & 1.7 & 1.7 & 1.6 & 1.6 & 1.7 & 1.8 \\
\hline 11 & 3.1 & 3.8 & 2.8 & 2.8 & 2.9 & 2.9 & 3.0 & 3.0 & 3.1 & 2.8 & 2.8 & 2.6 & 2.2 & 2.5 & 2.6 \\
\hline Ap & 0.8 & 0.9 & 0.8 & 0.7 & 0.7 & 0.5 & 0.5 & 0.5 & 0.5 & 0.5 & 0.5 & 0.5 & 0.4 & 0.5 & 0.5 \\
\hline
\end{tabular}

aNormative values based on $\mathrm{Fe}_{2} \mathrm{O}_{3} / \mathrm{FeO}$ ratio of 0.15 . $\mathrm{Fe}_{2} \mathrm{O}_{3}$ is total iron as $\mathrm{Fe}_{2} \mathrm{O}_{3}$. CeN $/ \mathrm{Y}_{\mathrm{N}}$ represents ratio of chondrite-normalized values. 


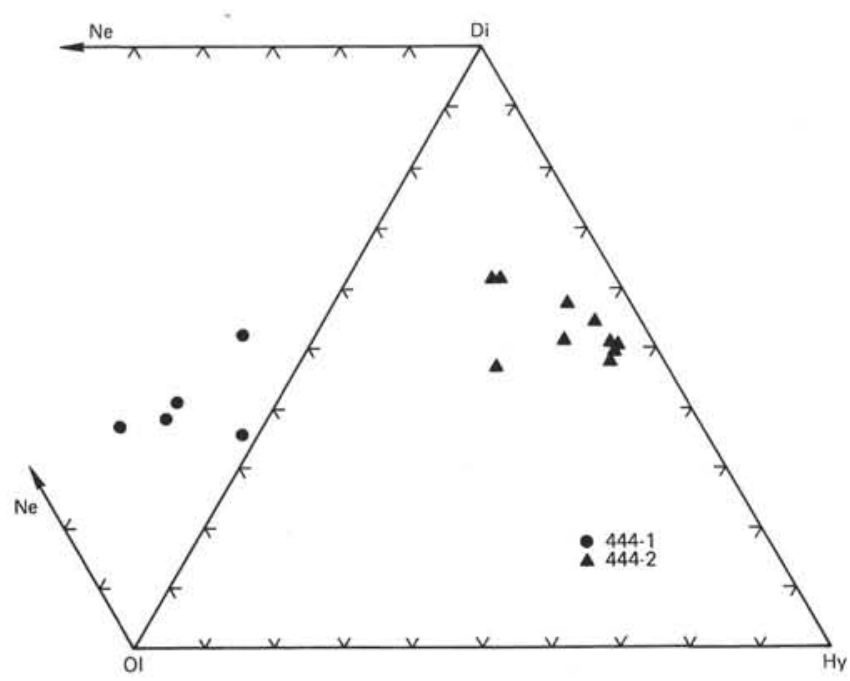

Figure 28. Normative nepheline (Ne), olivine $(\mathrm{Ol})$, diopside (Di), hypersthene (Hy) tetrahedron for Site 444. Normative values based on $\mathrm{Fe}_{2} \mathrm{O}_{3} / \mathrm{FeO}$ ratio of 0.15 .

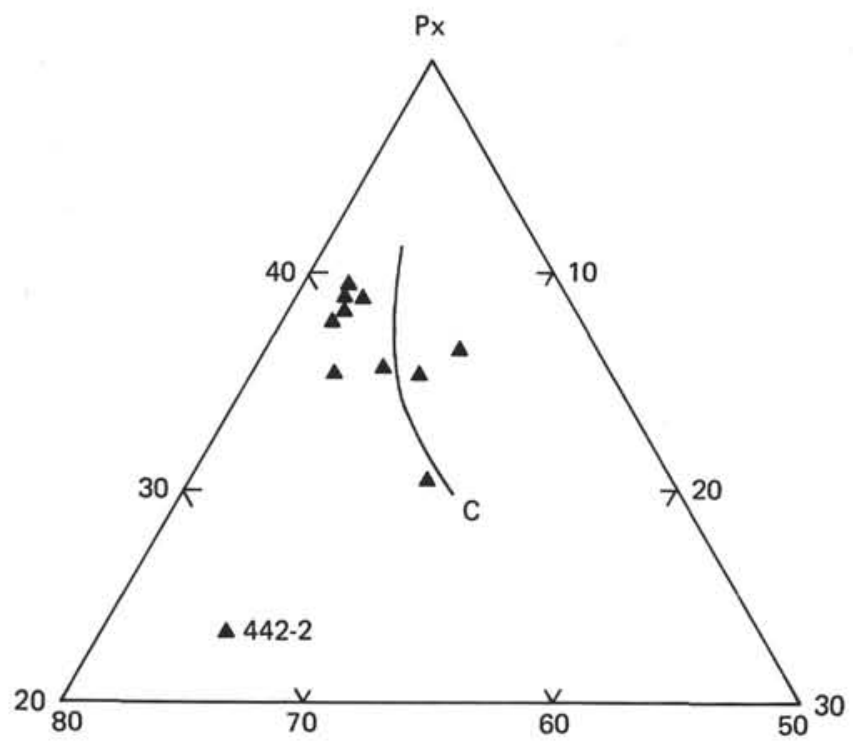

PI

OI

Figure 29. Normative plagioclase-pyroxene-olivine diagram for basalts from geochemical unit 444-2 (Site 444). The Fo-An cotectic (C) is from Shido et al. (1971).

tain hygromagmatophile elements (particularly $\mathrm{K}, \mathrm{Rb}$, $\mathrm{Sr}, \mathrm{Ba}, \mathrm{Ti}, \mathrm{Ce}$, and $\mathrm{La}$ ), relative to the tholeiitic group. Note also that units 446-1, 4, and 5 contain kaersutite.

Intra-unit variation is well-illustrated by the range of $\mathrm{Fe} / \mathrm{Mg}$ ratios (Figure 36 ) or, even better, by the $\mathrm{Ni} / \mathrm{Zr}$ ratio (Figure 38). With the exception of geochemical unit 446-5, it is apparent from the high $\mathrm{Fe} / \mathrm{Mg}$ ratios (generally in excess of 2.5), the low $\mathrm{Ni}$ abundances (less

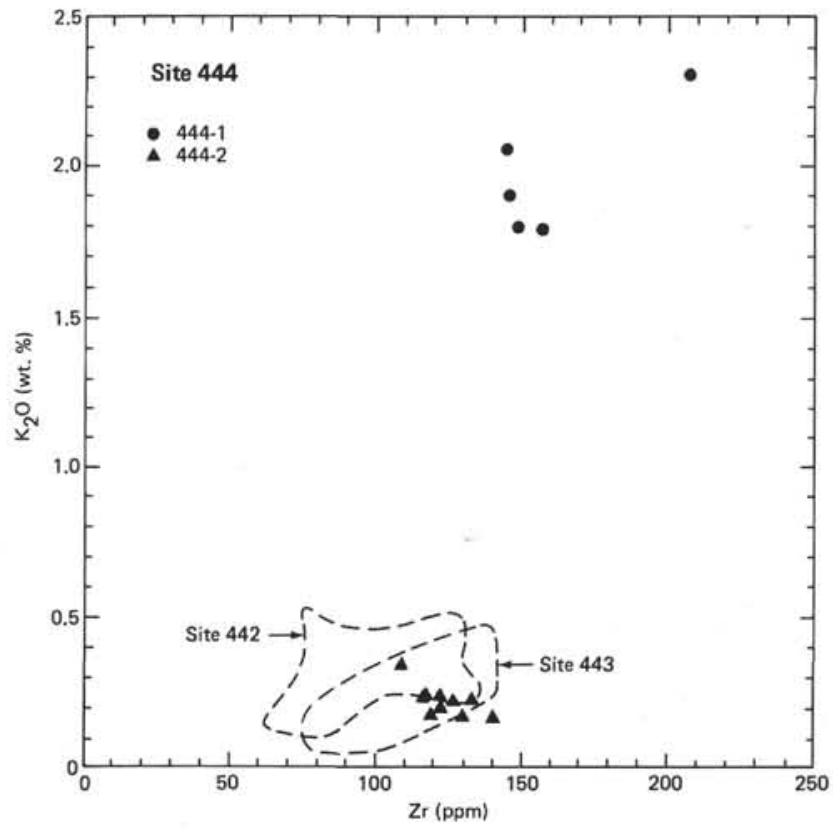

Figure 30. $\mathrm{K}_{2} \mathrm{O}$ versus $\mathrm{Zr}$, Site 444. Field for Site 442 from Figure 11; field for Site 443 from Figure 22.

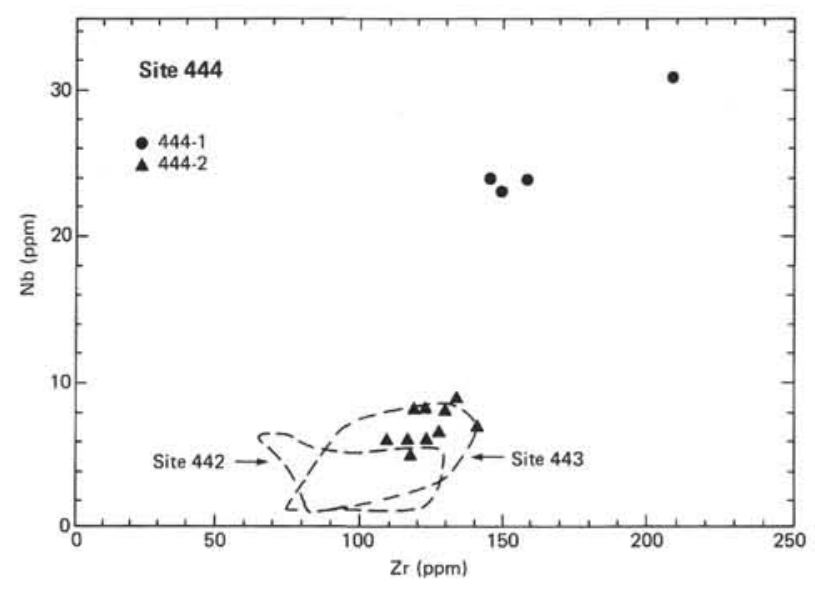

Figure 31. Nb versus $\mathrm{Zr}$, Site 444. Fields for Sites 442 and 443 from Figure 9.

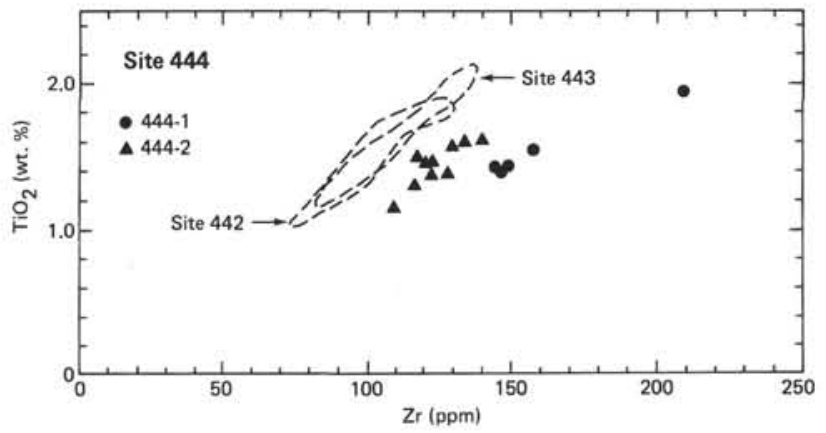

Figure 32. $\mathrm{TiO}_{2}$ versus $\mathrm{Zr}$, Site 444. Field for Site 442 from Figure 7; field for Site 443 from Figure 19. 


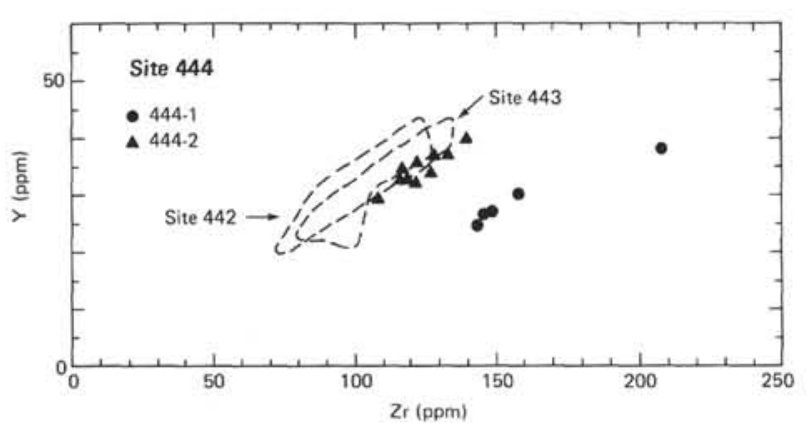

Figure 33. Y versus Zr, Site 444. Field for Site 442 from Tables 2 and 3; field for Site 443 from Figure 20.

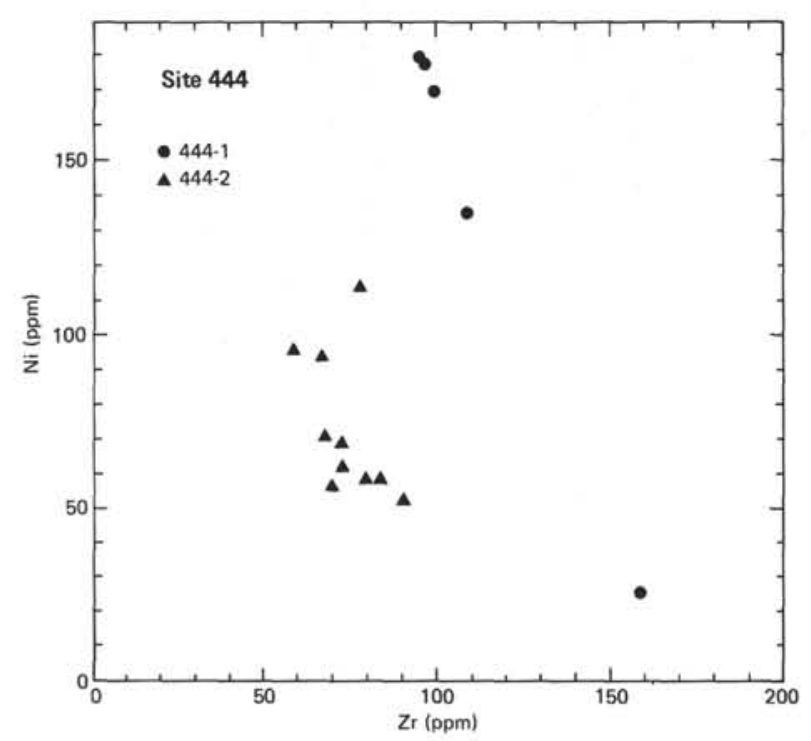

Figure 34. Ni versus $\mathrm{Zr}$, Site 444.

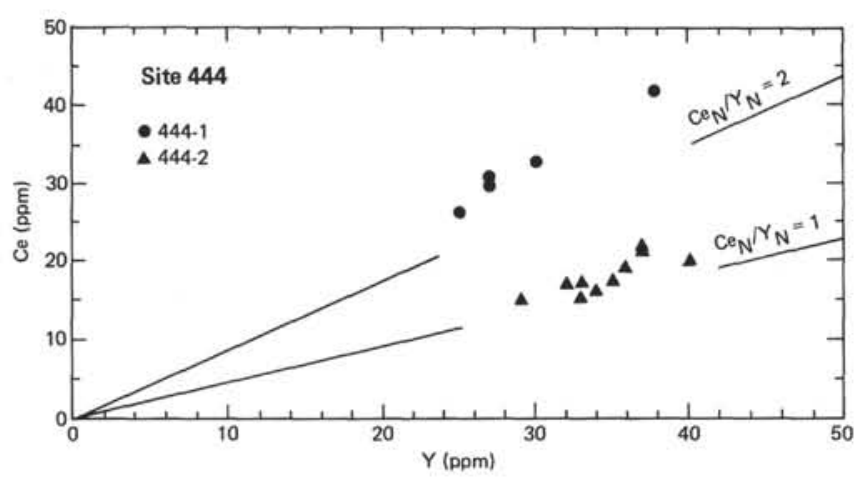

Figure 35. Ce versus $Y$, Site 444.

than $100 \mathrm{ppm}$, decreasing to less than $50 \mathrm{ppm}$ in units $446-1,3,4,7 \mathrm{a}, 7 \mathrm{~b}, 8,9,10 \mathrm{a}, 10 \mathrm{~b}, 12$, and 15), and the low $\mathrm{Cr}$ abundances (generally less than $150 \mathrm{ppm}$ ) that the basalts of Site 446 have undergone extensive fractionation and that they do not represent primary liquids. Unit 446-5, however, has significantly lower $\mathrm{Fe} / \mathrm{Mg}$ ratios $(0.90-1.68)$ and concomitant high $\mathrm{MgO}$ (7.3-17.7\%), high $\mathrm{Ni}(90-627 \mathrm{ppm})$, and high $\mathrm{Cr}$ (305-1206 $\mathrm{ppm}$ ) contents. The consanguineous nature of the 446-5 suite is clearly illustrated on the $\mathrm{Ni} / \mathrm{Zr}$ plot (Figure 38), and on several of the hygromagmatophile-element plots. High contents of altered olivine crystals (up to $30 \%$ ) in parts of unit $446-5$ strongly suggest that cumulus olivine may have caused the strong variation within this unit. However, implicit in the data is that cumulus chrome spinel, or pyroxene, also played an important role, because $\mathrm{Cr}$ increases in proportion to $\mathrm{Ni}$ (Table 9); olivine contains very little $\mathrm{Cr}$. Units $446-2,3$, and $7 \mathrm{~b}$ also show within-unit variation on the $\mathrm{Ni} / \mathrm{Zr}$ diagram, although in units $446-2$ and $446-3 \mathrm{Ni}$ (and $\mathrm{Cr}$ ) is practically invariant (Figure 38). This suggests that fractional crystallization involving olivine or pyroxene has not played an important role in determining the abundance variation of $\mathrm{Zr}$ (and other HYG elements) in units 446-2 and 446-3.

Although the high contents of many of the HYG elements are in part due to the fractionated nature of the 446 basalts, it is also apparent that many HYG-element ratios are very different between the tholeitic and alkalic groups, and between the Site 446 and Shikoku Basin basalts (apart from unit 444-1, which resembles the alkaline groups of 446). This is illustrated by plotting various HYG elements against $\mathrm{Zr}$ (Figures 39 to 45).

The alkalic basalt group has high $\mathrm{K} / \mathrm{Zr}, \mathrm{Ba} / \mathrm{Zr}$, $\mathrm{Rb} / \mathrm{Zr}$, and $\mathrm{Sr} / \mathrm{Zr}$ ratios compared with the tholeiitic group. The $\mathrm{Ba} / \mathrm{Zr}$ ratio, for example (Figure 39), is generally between 0.98 and 2.08 in the former, and between 0.38 and 1.08 in the latter $(\mathrm{Ba} / \mathrm{Zr}$ is less than 0.4 in the least-altered samples from the Shikoku Basin, although the upper sill, unit $444-1$, has a $\mathrm{Ba} / \mathrm{Zr}$ ratio of $0.85-1.04$; see Table 7).

$\mathrm{Ti} / \mathrm{Zr}$ ratios (Figure 40 ) are high in all Site 446 basalts, especially so in the alkalic group; however, this is unlike unit 444-1, which has low $\mathrm{Ti} / \mathrm{Zr}$ ratios (56-59). The alkalic group has the highest $\mathrm{Ti} / \mathrm{Zr}$ ratios (up to 163 ) of any basalts recovered during Leg 58, a reflection of the high ilmenite and $\mathrm{TiO}_{2}$ contents of these samples. The alkalic group also has the highest $\mathrm{Ce}_{\mathrm{N}} / \mathrm{Y}_{\mathrm{N}}$ ratios (4.09-5.92; Figure 41), although the tholeiitic group also exhibit relative light-REE enrichment $\left(\mathrm{Ce}_{\mathrm{N}} / \mathrm{Y}_{\mathrm{N}}\right.$ over 3). However, it is not only the light-REE contents which are high; it is apparent from the REE patterns for the Site 446 basalts (Wood et al., this volume) that the heavy REE, at least in the alkalic group, are significantly lower than in the Shikoku Basin basalts, even though the Site 446 rocks have undergone extensive fractionation. Thus, although the $\mathrm{Y} / \mathrm{Zr}$ ratios of the alkalic and tholeiitic suites are similar $(0.12-0.17$; Figure 42$)$, they are significantly lower than those of most of the Shikoku Basin basalts (0.3-0.4), apart from unit 444-1 (0.18).

All Site 446 basalts have low $\mathrm{Zr} / \mathrm{Nb}$ ratios (less than 12); in particular, units $446-4,5$, and $7 \mathrm{a}$ have $\mathrm{Zr} / \mathrm{Nb}$ ratios less than 7 (Figure 43). Low $\mathrm{Zr} / \mathrm{Nb}$ ratios are characteristic of "enriched" or "plume"-type basalts presently being erupted along certain sections of the MidAtlantic Ridge (e.g., $45^{\circ} \mathrm{N}$; Erlank and Kable, 1976; Tarney et al., 1979; Wood et al., 1979), on ocean islands (e.g., Iceland; Wood et al., in press), and in intracontinental alkali-basalt provinces (e.g., East African Rift; Weaver et al., 1972). The Ce/ $\mathrm{Zr}$ ratios of the Site 


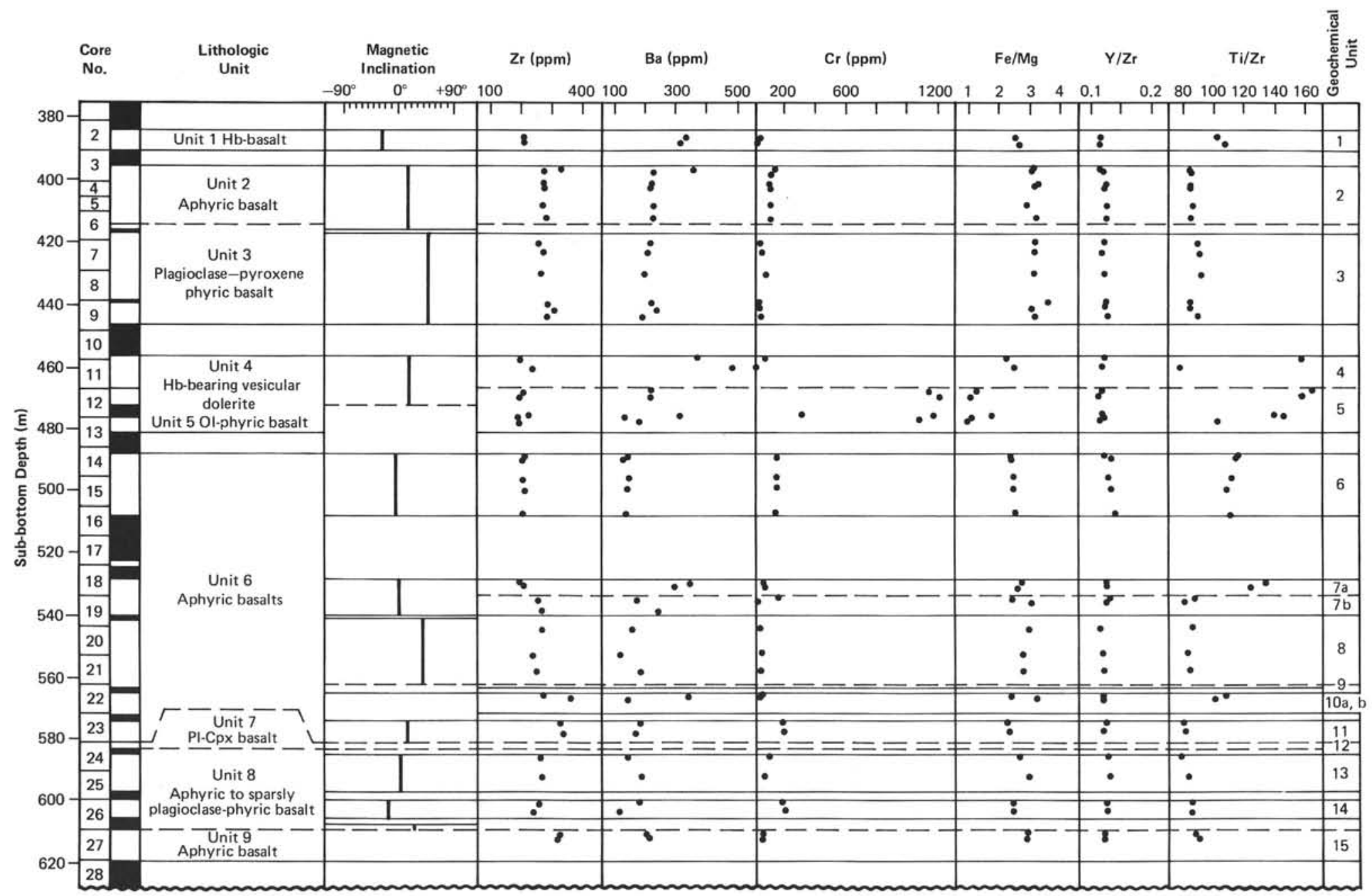

Figure 36. Down-hole variation in lithology, magnetic inclination, and selected geochemical parameters, Site 446. Shaded areas represent sediment. 
TABLE 8

Analyses, Element Ratios, and CIPW Norms for Basalts from Hole $446^{a}$

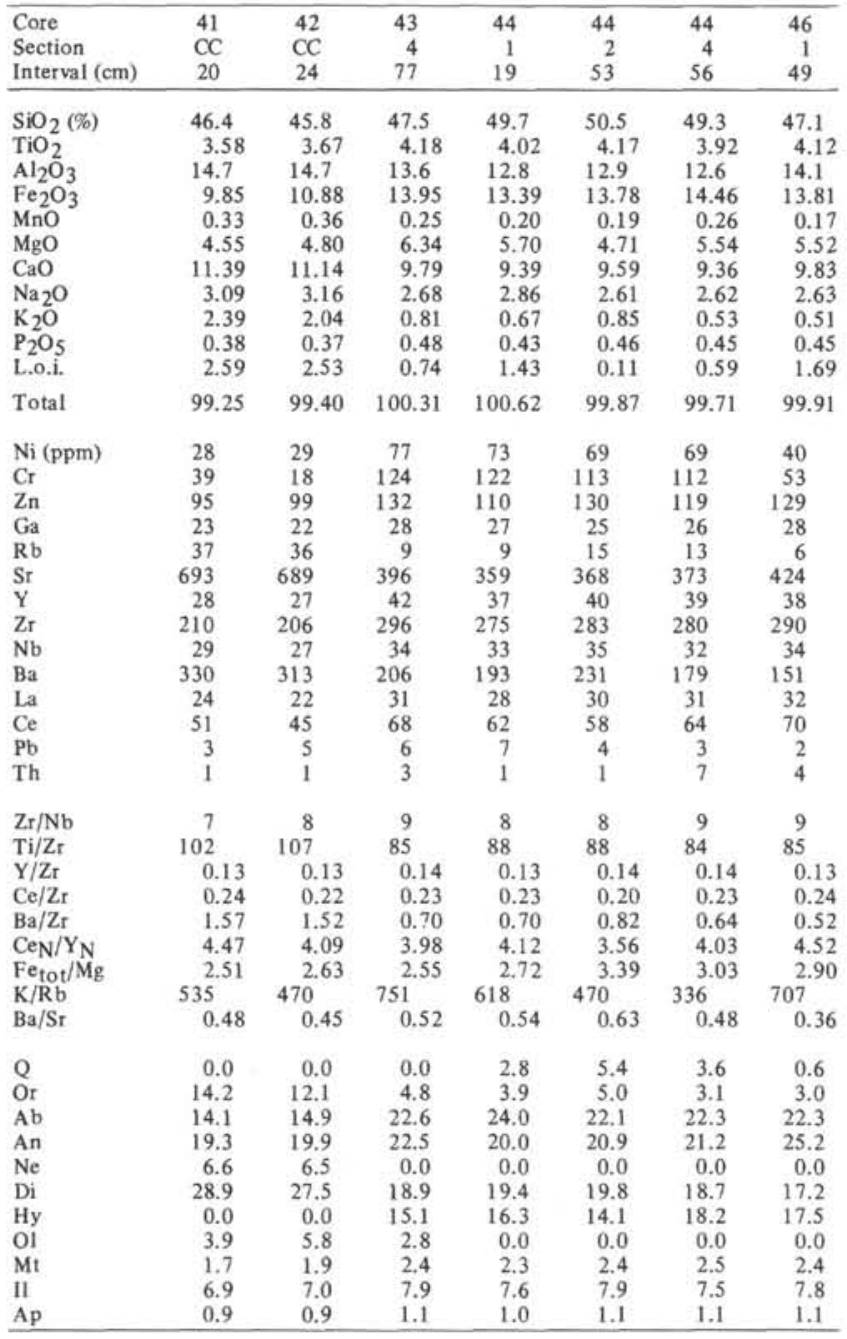

a Normative values based on $\mathrm{Fe}_{2} \mathrm{O}_{3} / \mathrm{FeO}$ ratio of 0.15 . $\mathrm{Fe}_{2} \mathrm{O}_{3}$ is total iron as $\mathrm{Fe}_{2} \mathrm{O}_{3}$. $\mathrm{Ce}_{\mathrm{N}} / \mathrm{Y}_{\mathrm{N}}$ represents ratio of chondrite-normalized values.

446 basalts are also characteristic of E-type ocean-ridge basalts (Figure 44) ( 0.3; Tarney et al., 1979), being significantly higher than in the Shikoku Basin basalts (less than 0.2 ), or in T-type and $\mathrm{N}$-type ocean-ridge basalts.

Units 446-4, 5, and $7 \mathrm{a}$ have high $\mathrm{P} / \mathrm{Zr}$ ratios (higher than 10; Figure 45), unlike the other units at Site 446, and unlike the basalts recovered from the Shikoku Basin. The wide variation in $\mathrm{P} / \mathrm{Zr}$ ratios within unit 446-5 basalts may be due to the presence of cumulus apatite, although the high phorphorus content in the alkalic units must be a function of the melt (and probably the source) composition. Note, however, that the other alkalic units-446-1 and 446-10a-have $\mathrm{P} / \mathrm{Zr}$ ratios similar to the tholeiitic suites, although there are slight differences in the $\mathrm{P} / \mathrm{Zr}$ ratios of the various units.

The processes giving rise to the inter-unit chemical variations at Site 446 are difficult to quantify without detailed major- and trace-element modeling. A compre- hensive analytical survey of the constituent minerals is necessary before this can be attempted. It is apparent, however, that the two main compositional types-alkalic basalts and tholeiitic basalts-cannot easily be related by fractional crystallization, because of the significant differences in the $\mathrm{Zr} / \mathrm{Nb}$, $\mathrm{Th} / \mathrm{Hf}$ (Wood et al., this volume), and alkali-elements $/ \mathrm{Zr}$ ratios of the two suites. In addition, it may be difficult to relate the two suites by varying degrees of partial melting of a compositionally homogeneous source, although further modeling is necessary to ascertain this.

\section{SUMMARY AND DISCUSSION}

One of the objectives of Leg 58 was to obtain samples of basaltic basement from the Shikoku and Daito Basins, in order to compare the petrological and chemical characteristics of this basement with those of other marginal basins and of the major ocean basins. Unfortunately, although substantial amounts of basalt were recovered at all sites except 445 , only drilling at Site 442 and possibly Site 443 penetrated basalt belonging unequivocally to oceanic layer 2 ; drilling at all the remaining sites recovered post-basement basaltic material which may represent, in part, off-axis activity. It is unlikely, however, that the sill and flow emplacement at Sites 442,443 , and 444 post-dates true basement by significantly more than 2 m.y. $(80 \mathrm{~km}$ from the ridge axis at a spreading rate of $4 \mathrm{~cm} / \mathrm{yr}$ ), and for the purposes of the present discussion it is considered that this "off-axis" activity tapped a mantle source similar to that which gave rise to the underlying basement.

The crustal structure of the Shikoku Basin, and the composition of the basalts recovered during Leg 58, support the suggestion of Karig (1971) that the majority of the western Pacific marginal basins are extensional and formed by sea-floor spreading broadly analogous to that occurring in the major ocean basins. The Shikoku Basin basalts exhibit considerable chemical variation, which may be due to a combination of several processes: low-temperature and hydrothermal alteration by sea water; within-flow and within-sill differentiation; highlevel fractionation in sub-ridge magma chambers; varying degrees of partial melting under varying $P, P_{\mathrm{H}_{2} \mathrm{O}}$, and $P_{\mathrm{O}_{2}}$ conditions, hence different stable-phase assemblages during partial melting; and heterogeneity in the mantle source beneath the ridge axis. It is important to assess these effects before making any regional comparisons with basalts from different tectonic environments, and before evaluating the significance of basalt chemistry on the mechanisms of back-arc spreading.

It is apparent from Figures 10 to 14 and 22 to 24 that the compositions of the basalts recovered from Sites 442 and 443 have been modified by sea water alteration, resulting in variably increased concentrations of $\mathrm{K}, \mathrm{Rb}$, $\mathrm{P}_{2} \mathrm{O}_{5}, \mathrm{Sr}$, and to a lesser extent $\mathrm{Ba}$. This limits the use of these elements in any petrogenetic discussions, although the low abundances of $\mathrm{K}$ and $\mathrm{Rb}$ relative to $\mathrm{Zr}$ in some of the fresher samples do suggest that the magmas probably had low $\mathrm{K} / \mathrm{Zr}$ and $\mathrm{Rb} / \mathrm{Zr}$ ratios originally. In addition, $\mathrm{Ba} / \mathrm{Zr}$ ratios are consistently low, which corroborates previous evidence (Hart et al., 1974) that $\mathrm{Ba}$ is less 
TABLE 9

Analyses, Element Ratios, and CIPW Norms for Basalts from Hole $446 \mathrm{~A}^{\mathrm{a}}$

\begin{tabular}{|c|c|c|c|c|c|c|c|c|c|c|c|c|c|}
\hline $\begin{array}{l}\text { Core } \\
\text { Section } \\
\text { Interval (cm) }\end{array}$ & $\begin{array}{r}3 \\
3 \\
35\end{array}$ & $\begin{array}{r}3 \\
4 \\
33\end{array}$ & $\begin{array}{r}4 \\
1 \\
39\end{array}$ & $\begin{array}{r}4 \\
1 \\
139\end{array}$ & $\begin{array}{r}5 \\
2 \\
26\end{array}$ & $\begin{array}{r}6 \\
2 \\
34\end{array}$ & $\begin{array}{r}7 \\
1 \\
48\end{array}$ & $\begin{array}{r}7 \\
3 \\
97\end{array}$ & $\begin{array}{r}8 \\
1 \\
66\end{array}$ & $\begin{array}{r}9 \\
1 \\
79\end{array}$ & $\begin{array}{r}9 \\
2 \\
120\end{array}$ & $\begin{array}{r}9 \\
3 \\
97\end{array}$ & $\begin{array}{r}10 \\
5 \\
107\end{array}$ \\
\hline $\mathrm{SiO}_{2}(\%)$ & 48.3 & 50.7 & 50.4 & 50.7 & 49.5 & 50.9 & 51.1 & 50.5 & 50.6 & 49.8 & 50.8 & 47.5 & 44.8 \\
\hline $\mathrm{TiO}_{2}$ & 4.62 & 3.88 & 3.89 & 3.90 & 3.92 & 3.91 & 3.82 & 4.07 & 3.92 & 4.02 & 4.23 & 4.16 & 4.90 \\
\hline $\mathrm{Al}_{2} \mathrm{O}_{3}$ & 14.8 & 12.5 & 12.5 & 12.6 & 12.5 & 12.6 & 13.1 & 13.1 & 13.0 & 12.7 & 12.8 & 12.9 & 13.3 \\
\hline $\mathrm{Fe}_{2} \mathrm{O}_{3}$ & 12.10 & 14.23 & 14.18 & 14.03 & 14.46 & 14.22 & 13.83 & 13.39 & 13.38 & 13.53 & 13.48 & 15.19 & 11.85 \\
\hline $\mathrm{MnO}$ & 0.27 & 0.19 & 0.18 & 0.22 & 0.22 & 0.18 & 0.15 & 0.16 & 0.16 & 0.24 & 0.20 & 0.21 & 0.19 \\
\hline $\mathrm{MgO}$ & 4.48 & 5.40 & 5.11 & 5.22 & 5.76 & 5.09 & 5.09 & 4.88 & 5.00 & 4.44 & 5.16 & 5.55 & 6.25 \\
\hline $\mathrm{CaO}$ & 9.82 & 9.34 & 9.39 & 9.45 & 9.68 & 9.25 & 8.34 & 9.34 & 9.37 & 9.45 & 8.28 & 9.71 & 12.44 \\
\hline $\mathrm{Na}_{2} \mathrm{O}$ & 3.05 & 2.42 & 2.49 & 2.53 & 2.48 & 2.55 & 2.58 & 2.57 & 2.80 & 2.70 & 2.69 & 2.49 & 2.81 \\
\hline $\mathrm{K}_{2} \mathrm{O}$ & 1.31 & 0.84 & 0.83 & 0.81 & 0.56 & 0.91 & 0.90 & 0.79 & 0.61 & 0.92 & 0.92 & 0.55 & 1.55 \\
\hline $\mathrm{P}_{2} \mathrm{O}_{5}$ & 0.53 & 0.44 & 0.44 & 0.45 & 0.45 & 0.45 & 0.40 & 0.42 & 0.41 & 0.45 & 0.52 & 0.47 & 0.99 \\
\hline L.o.i. & 0.60 & 0.06 & 0.24 & 0.24 & 0.38 & 0.04 & 1.11 & 0.72 & 0.71 & 1.60 & 1.88 & 0.69 & 0.48 \\
\hline Total & 99.90 & 99.98 & 99.59 & 100.06 & 99.98 & 100.10 & 100.43 & 99.93 & 99.95 & 99.81 & 100.96 & 99.39 & 99.53 \\
\hline $\mathrm{Ni}$ & 80 & 72 & 67 & 62 & 65 & 69 & 34 & 41 & 46 & 33 & 38 & 39 & 45 \\
\hline $\mathrm{Cr}$ & 133 & 115 & 105 & 111 & 114 & 115 & 46 & 54 & 81 & 39 & 40 & 40 & 69 \\
\hline $\mathrm{Zn}$ & 147 & 129 & 127 & 123 & 128 & 122 & 127 & 140 & 108 & 119 & 135 & 129 & 98 \\
\hline Ga & 29 & 24 & 25 & 25 & 24 & 24 & 25 & 27 & 27 & 26 & 28 & 24 & 24 \\
\hline $\mathrm{Rb}$ & 19 & 20 & 15 & 15 & 9 & 21 & 15 & 12 & 9 & 13 & 13 & 6 & 22 \\
\hline Sr & 423 & 359 & 365 & 361 & 375 & 357 & 377 & 398 & 388 & 390 & 405 & 409 & 1088 \\
\hline Y & 44 & 39 & 40 & 40 & 40 & 41 & 37 & 36 & 36 & 40 & 43 & 41 & 26 \\
\hline $\mathrm{Zr}$ & 328 & 271 & 275 & 279 & 269 & 277 & 258 & 270 & 258 & 286 & 305 & 281 & 188 \\
\hline $\mathrm{Nb}$ & 38 & 32 & 35 & 34 & 36 & 34 & 32 & 31 & 30 & 32 & 35 & 30 & 32 \\
\hline $\mathrm{Ba}$ & 354 & 230 & 222 & 219 & 226 & 227 & 215 & 206 & 196 & 213 & 232 & 184 & 362 \\
\hline $\mathrm{La}$ & 34 & 28 & 31 & 29 & 31 & 28 & 27 & 28 & 27 & 29 & 32 & 29 & 25 \\
\hline $\mathrm{Ce}$ & 74 & 63 & 62 & 67 & 67 & 69 & 62 & 66 & 57 & 64 & 74 & 68 & 58 \\
\hline $\mathrm{Pb}$ & 4 & 4 & 4 & 7 & 8 & 2 & 4 & 7 & 2 & 3 & 6 & 5 & 4 \\
\hline Th & $<1$ & 3 & 2 & 3 & 7 & 1 & 3 & 4 & 3 & 3 & 1 & 4 & $<1$ \\
\hline $\mathrm{Zr} / \mathrm{Nb}$ & 9 & 8 & 8 & 8 & 7 & 8 & 8 & 9 & 9 & 9 & 9 & 9 & 6 \\
\hline $\mathrm{Ti} / \mathrm{Zr}_{\mathrm{r}}$ & 84 & 86 & 85 & 84 & 87 & 85 & 89 & 90 & 91 & 84 & 83 & 89 & 156 \\
\hline $\mathrm{Y} / \mathrm{Zr}$ & 0.13 & 0.14 & 0.15 & 0.14 & 0.15 & 0.15 & 0.14 & 0.13 & 0.14 & 0.14 & 0.14 & 0.15 & 0.14 \\
\hline $\mathrm{Ce} / \mathrm{Zr}$ & 0.23 & 0.23 & 0.23 & 0.24 & 0.25 & 0.25 & 0.24 & 0.24 & 0.22 & 0.22 & 0.24 & 0.24 & 0.31 \\
\hline $\mathrm{Ba} / \mathrm{Zr}$ & 1.08 & 0.85 & 0.81 & 0.78 & 0.84 & 0.82 & 0.83 & 0.76 & 0.76 & 0.74 & 0.76 & 0.65 & 1.93 \\
\hline${ }^{C} e_{N} / Y_{N}$ & 4.13 & 3.97 & 3.81 & 4.11 & 4.11 & 4.13 & 4.12 & 4.50 & 3.89 & 3.93 & 4.23 & 4.07 & 5.48 \\
\hline $\mathrm{Fe}_{\text {tot }} / \mathrm{Mg}$ & 3.13 & 3.06 & 3.22 & 3.12 & 2.91 & 3.24 & 3.15 & 3.18 & 3.10 & 3.54 & 3.03 & 3.17 & 2.20 \\
\hline $\mathrm{K} / \mathrm{R} \mathrm{b}$ & 573 & 348 & 458 & 447 & 515 & 360 & 498 & 549 & 559 & 584 & 589 & 756 & 586 \\
\hline $\mathrm{Ba} / \mathrm{Sr}$ & 0.84 & 0.64 & 0.61 & 0.61 & 0.60 & 0.64 & 0.57 & 0.52 & 0.51 & 0.55 & 0.57 & 0.45 & 0.33 \\
\hline Q & 0.0 & 5.4 & 5.3 & 5.2 & 3.8 & 5.3 & 6.2 & 5.7 & 5.0 & 4.7 & 6.0 & 1.5 & 0.0 \\
\hline Or & 7.8 & 5.0 & 4.9 & 4.8 & 3.3 & 5.4 & 5.3 & 4.7 & 3.6 & 5.4 & 5.4 & 3.2 & 9.2 \\
\hline $\mathrm{Ab}$ & 25.8 & 20.5 & 21.2 & 21.4 & 21.0 & 21.5 & 21.7 & 21.8 & 23.7 & 22.9 & 22.5 & 21.2 & 14.8 \\
\hline An & 22.9 & 20.7 & 20.4 & 20.5 & 21.4 & 20.2 & 21.5 & 21.8 & 21.1 & 19.8 & 20.0 & 22.6 & 19.2 \\
\hline $\mathrm{Ne}$ & 0.0 & 0.0 & 0.0 & 0.0 & 0.0 & 0.0 & 0.0 & 0.0 & 0.0 & 0.0 & 0.0 & 0.0 & 4.9 \\
\hline Di & 18.5 & 18.9 & 19.5 & 19.5 & 19.7 & 18.9 & 14.3 & 18.2 & 18.9 & 20.2 & 14.4 & 19.0 & 29.8 \\
\hline Hy & 10.6 & 17.3 & 16.2 & 16.3 & 18.1 & 16.4 & 18.2 & 14.9 & 15.1 & 13.2 & 17.1 & 18.7 & 0.0 \\
\hline O1 & 0.5 & 0.0 & 0.0 & 0.0 & 0.0 & 0.0 & 0.0 & 0.0 & 0.0 & 0.0 & 0.0 & 0.0 & 6.9 \\
\hline $\mathrm{Mt}$ & 2.1 & 2.5 & 2.5 & 2.4 & 2.5 & 2.5 & 2.4 & 2.3 & 2.3 & 2.4 & 2.3 & 2.7 & 2.1 \\
\hline II & 8.8 & 7.4 & 7.4 & 7.4 & 7.4 & 7.4 & 7.2 & 7.7 & 7.5 & 7.7 & 8.0 & 8.0 & 9.3 \\
\hline Ap & 1.3 & 1.0 & 1.1 & 1.1 & 1.1 & 1.1 & 0.9 & 1.0 & 1.0 & 1.1 & 1.2 & 1.1 & 2.4 \\
\hline
\end{tabular}

mobile than $\mathrm{K}, \mathrm{Rb}$, or $\mathrm{Sr}$ during low-temperature alteration by sea water. Halmyrolytic and hydrothermal activity appear to have been less important in affecting the chemistry of the Site 444 (especially unit 444-1) and Site 446 basalts, where $\mathrm{K}, \mathrm{Rb}, \mathrm{Ba}$, and $\mathrm{Sr}$ behave in a coherent fashion. This may be a function of the generally much higher abundances of these elements at these sites. As a consequence of the alteration at Sites 442 and 443, we shall restrict our discussion primarily to those elements which are considered immobile during hydrothermal and halmyrolitic activity-particularly $\mathrm{Ti}, \mathrm{Zr}, \mathrm{Nb}$, Y, Ni, and Cr (e.g., Pearce and Cann, 1971, 1973; Pearce, 1975), and the REE, although noting the possibility of Ce mobility illustrated by Ludden and Thompson(1979).

Mineralogically, the basalts from Site 442 and 443 and unit 444-2 closely resemble abyssal basalts recovered from the major ocean basins. However, one important difference is the exceptionally high vesicularity of all the Site 442 basalts, and some of the Site 443 and 444 basalts. It is not unusual to find vesicular basalts on mid-ocean ridges, but vesicles are rare at the depths from which the basalts at Sites 442 (4600 meters), 443 (4400 meters), and 444 (4800 meters) were recovered, because of the inverse relationship between depth of extrusion and degree of vesicularity. It could be suggested that the basalts were emplaced in shallow water, before undergoing subsidence to their present depth, but this is considered unlikely. First, the basalts would have to have been emplaced in very shallow water (less than 500 meters) to produce the observed degree of vesicularity, but the sedimentary evidence indicates that the depth of emplacement was in fact close to the carbonate-compensation depth ( $\sim 4000$ meters, near the present depth). Second, at Site 443 at least, not all the basalts are vesicular.

It is more likely that the high vesicularity is a result of a high volatile content in the magmas. Two suites of ba- 
TABLE 9 - Continued

\begin{tabular}{|c|c|c|c|c|c|c|c|c|c|c|c|c|c|c|}
\hline $\begin{array}{r}11 \\
2 \\
27\end{array}$ & $\begin{array}{r}12 \\
1 \\
59\end{array}$ & $\begin{array}{r}12 \\
2 \\
72\end{array}$ & $\begin{array}{r}12 \\
4 \\
36\end{array}$ & $\begin{array}{r}12 \\
4 \\
69\end{array}$ & $\begin{array}{r}13 \\
1 \\
109\end{array}$ & $\begin{array}{r}14 \\
1 \\
92\end{array}$ & $\begin{array}{r}14 \\
3 \\
64\end{array}$ & $\begin{array}{r}15 \\
1 \\
70\end{array}$ & $\begin{array}{r}15 \\
3 \\
75\end{array}$ & $\begin{array}{r}16 \\
2 \\
72\end{array}$ & $\begin{array}{r}18 \\
4 \\
85\end{array}$ & $\begin{array}{r}18 \\
5 \\
32\end{array}$ & $\begin{array}{r}19 \\
2 \\
54\end{array}$ & $\begin{array}{r}19 \\
4 \\
7\end{array}$ \\
\hline 48.5 & 42.4 & 41.4 & 45.6 & 42.8 & 41.4 & 47.2 & 49.6 & 49.6 & 49.3 & 49.7 & 46.9 & 46.1 & 47.4 & 49.5 \\
\hline 2.97 & 4.07 & 3.60 & 3.76 & 3.10 & 2.26 & 3.77 & 3.61 & 3.58 & 3.60 & 3.60 & 4.17 & 4.08 & 3.45 & 3.98 \\
\hline 14.3 & 10.3 & 9.4 & 12.2 & 9.8 & 9.1 & 13.6 & 13.0 & 13.0 & 13.2 & 13.2 & 13.4 & 13.7 & 13.9 & 12.9 \\
\hline 10.37 & 12.77 & 13.69 & 10.59 & 13.46 & 13.68 & 13.41 & 13.01 & 13.10 & 13.07 & 12.30 & 13.37 & 12.84 & 12.56 & 13.64 \\
\hline 0.19 & 0.22 & 0.27 & 0.16 & 0.25 & 0.35 & 0.19 & 0.17 & 0.17 & 0.16 & 0.19 & 0.17 & 0.18 & 0.19 & 0.20 \\
\hline 4.89 & 12.43 & 15.43 & 7.30 & 15.03 & 17.69 & 6.62 & 6.33 & 6.32 & 6.35 & 5.80 & 5.82 & 5.93 & 6.18 & 5.31 \\
\hline 9.18 & 11.25 & 9.09 & 12.49 & 9.28 & 8.12 & 10.30 & 9.88 & 9.87 & 9.99 & 10.39 & 9.93 & 10.34 & 10.08 & 9.53 \\
\hline 3.74 & 1.49 & 1.13 & 2.42 & 1.20 & 0.87 & 2.65 & 2.69 & 2.60 & 2.50 & 2.65 & 3.01 & 2.94 & 3.01 & 2.66 \\
\hline 1.95 & 0.74 & 0.67 & 1.19 & 0.57 & 0.25 & 0.55 & 0.27 & 0.45 & 0.42 & 0.41 & 1.33 & 1.05 & 0.69 & 0.35 \\
\hline 1.38 & 0.73 & 0.69 & 0.63 & 0.50 & 0.36 & 0.35 & 0.34 & 0.34 & 0.34 & 0.34 & 0.61 & 0.63 & 0.42 & 0.43 \\
\hline 1.49 & 2.88 & 4.11 & 3.53 & 4.18 & 5.61 & 1.13 & 1.07 & 1.22 & 1.08 & 1.46 & 1.18 & 1.68 & 1.63 & 1.17 \\
\hline 98.94 & 99.33 & 99.43 & 99.85 & 100.16 & 99.71 & 99.83 & 100.02 & 100.30 & 100.03 & 99.95 & 99.98 & 99.50 & 99.53 & 99.67 \\
\hline 0 & 293 & 496 & 90 & 563 & 627 & 77 & 72 & 72 & 72 & 74 & 38 & 49 & 76 & 31 \\
\hline 0 & 1137 & 1206 & 305 & 1169 & 1070 & 144 & 141 & 139 & 135 & 131 & 48 & 52 & 147 & 13 \\
\hline 112 & 80 & 94 & 80 & 86 & 90 & 122 & 115 & 105 & 113 & 104 & 88 & 114 & 120 & 114 \\
\hline 28 & 19 & 19 & 23 & 15 & 16 & 24 & 23 & 24 & 24 & 23 & 24 & 24 & 25 & 26 \\
\hline 30 & 16 & 16 & 21 & 14 & 7 & 8 & 1 & 3 & 2 & 5 & 14 & 12 & 10 & 8 \\
\hline 1553 & 480 & 418 & 724 & 230 & 233 & 379 & 367 & 368 & 373 & 374 & 673 & 720 & 385 & 446 \\
\hline 29 & 19 & 17 & 22 & 17 & 16 & 28 & 31 & 30 & 32 & 34 & 26 & 27 & 36 & 41 \\
\hline 229 & 150 & 138 & 163 & 129 & 134 & 198 & 194 & 194 & 202 & 198 & 191 & 200 & 243 & 302 \\
\hline 46 & 24 & 25 & 27 & 24 & 22 & 23 & 19 & 20 & 24 & 17 & 27 & 32 & 22 & 31 \\
\hline 476 & 212 & 210 & 304 & 127 & 172 & 137 & 121 & 139 & 133 & 128 & 334 & 284 & 161 & 228 \\
\hline 34 & 22 & 23 & 24 & 20 & 20 & 22 & 18 & 20 & 20 & 20 & 29 & 31 & 23 & 28 \\
\hline 80 & 42 & 41 & 44 & 38 & 38 & 44 & 42 & 39 & 41 & 43 & 61 & 64 & 50 & 65 \\
\hline 6 & 7 & 8 & 3 & 1 & 4 & 5 & 3 & 2 & 5 & 1 & $<1$ & 4 & 6 & 6 \\
\hline 5 & 5 & 2 & 1 & $<1$ & 2 & 2 & $<1$ & $<1$ & 2 & $<i$ & 2 & $<1$ & 3 & 2 \\
\hline 5 & 0 & 0 & 6 & 5 & 6 & 9 & 10 & 1 & 8 & 12 & 7 & 6 & 11 & 10 \\
\hline 78 & 163 & 156 & 138 & 144 & 101 & 114 & 112 & 111 & 107 & 109 & 131 & 122 & 85 & 79 \\
\hline 0.13 & 0.13 & 0.12 & 0.13 & 0.13 & 0.12 & 0.14 & 0.16 & 0.15 & 0.16 & 0.1 & 0.1 & 0.1 & 0.15 & 0.14 \\
\hline 0.35 & 0.28 & 0. & 0.27 & 0.29 & 0.28 & 0.22 & 0.22 & 0.2 & 0.2 & 0.2 & 0. & 0. & 0.21 & 0.22 \\
\hline 2.08 & 1.41 & 1.5 & 1.87 & 0. & 1.28 & 0.69 & 0.62 & 0. & 0. & 0. & 1. & 1. & 0.66 & 0.75 \\
\hline 6.78 & 5.43 & 5.92 & 4.91 & 5.49 & 5.83 & 3.86 & 3.33 & 3.1 & 3.1 & 3.1 & 5.76 & 5. & 3.41 & 3.89 \\
\hline 2.46 & 1.19 & 1.0 & 1.68 & 1.04 & 0.90 & 2.35 & 2.38 & 2.40 & 2.39 & 2.4 & 2.66 & 2.51 & 2.36 & 2.98 \\
\hline 540 & 383 & 348 & 469 & 341 & 302 & 575 & 2217 & 1257 & 1724 & 687 & 791 & 728 & 572 & 359 \\
\hline 0.31 & 0.44 & 0.50 & 0.42 & 0.55 & 0.74 & 0.36 & 0.33 & 0.38 & 0.36 & 0.34 & 0.50 & 0.39 & 0.42 & 0.51 \\
\hline 0.0 & 0. & & 0 & & & 0. & 3. & 2. & 2 & 3 & 0. & 0. & 0. & 4.8 \\
\hline 11.6 & & & 7 & & 1. & & 1. & & 2.5 & 2. & 7 & 6 & 4.1 & 2.1 \\
\hline 31.1 & 12.7 & 9.6 & 17.7 & 10.1 & 7. & 22.5 & 22.8 & 21.9 & 21.2 & 22. & 25 & 25 & 25.6 & 22.6 \\
\hline 16.7 & 19.4 & 18.7 & 19.0 & 19 & 20 & & & & 23 & 22 . & 19 & 21 & 22.4 & 22.2 \\
\hline 0.5 & 0. & 0 . & 1.6 & 0.0 & 0.0 & 0.0 & 0.0 & 0.0 & 0.0 & 0.0 & 0.0 & 0. & 0.0 & 0.0 \\
\hline 17.1 & 25.8 & 17.9 & 31.7 & 18.7 & 14. & 20.7 & 19.9 & 19.9 & 19. & 21.9 & 21.7 & 21. & 20.7 & 18.6 \\
\hline 0. & 2. & प. & 0 & 11.8 & & 13.1 & 18.0 & 18.0 & 18.3 & 14.8 & 1. & 1. & 8.4 & 16.4 \\
\hline 9.9 & 20.0 & 24.6 & 8. & 21.7 & 27.2 & 4.1 & 0.0 & 0.0 & 0.0 & 0. & 9. & 10.0 & 6.3 & 0.0 \\
\hline 1.8 & 2. & 2. & 1. & 2. & 2. & 2 & 2. & 2 & 2. & 2. & 2. & 2 & 2.2 & 2.4 \\
\hline 5.7 & 7 & 6.9 & 7. & 5. & 4 & 7. & 6 & 6. & 6. & 6. & 7. & 7 & 6.6 & 7.6 \\
\hline 3.3 & 1.7 & 1.6 & 1.5 & 1.2 & 0.9 & 0.8 & 0.8 & 0.8 & 0.8 & 0.8 & 1.4 & 1.5 & 1.0 & 1.0 \\
\hline
\end{tabular}

salts from the East Scotia Sea back-arc basin exhibit exceptionally high vesicularity (Saunders and Tarney, 1979), not unlike the Site 442 basalts. It has been suggested (Saunders and Tarney, 1979) that this vesicularity is due to high volatile contents in the basaltic magma and in the mantle source, possibly resulting from the penetration of hydrous fluids from the adjacent subduction zone. Subsequent studies of the East Scotia Sea basalt glasses have confirmed the hydrated nature of the vesicular basalts (Meunow et al., in press).

The suggestion that fluids arising from the subducted slab locally have contaminated the mantle source of the East Scotia Sea basalts is supported by the fact that $\mathrm{K}$, $\mathrm{Rb}, \mathrm{Sr}$, and $\mathrm{Ba}$ contents and ${ }^{87} \mathrm{Sr} / 86 \mathrm{Sr}$ ratios are correspondingly higher in the fresh vesicular basalts than in the non-vesicular basalts, these elements presumably being carried with fluids expelled from the slab. Unfortunately, because the Shikoku Basin basalts have undergone excessive alteration by sea water, their $\mathrm{K}, \mathrm{Rb}, \mathrm{Ba}$, and $\mathrm{Sr}$ contents cannot be used as supporting evidence. Nonetheless, the high vesicularity of many of the Shiko$\mathrm{ku}$ Basin basalts does indicate a high volatile content in the source region beneath the basin. Basalts dredged from the Mariana Trough, while not exhibiting the same degree of vesicularity as the Shikoku Basin basalts, do contain significantly more $\mathrm{H}_{2} \mathrm{O}+$ and $\mathrm{CO}_{2}$ than midocean-ridge basalts (Garcia et al., 1979). Available data suggest therefore that back-arc-basin basalts are derived from a mantle source with a locally increased volatile content, and it may be presumed that the source for these volatiles was an adjacent subduction zone (which since migrated eastward beneath what is now the Bonin Islands).

It is apparent that none of the Leg 58 basalts can be considered as primary mantle melts: their $\mathrm{Fe} / \mathrm{Mg}$ ratios are too high, and their $\mathrm{Cr}$ and $\mathrm{Ni}$ contents too low, for the melts to have been in equilibrium with predicted mantle compositions. Those basalts with high $\mathrm{MgO}, \mathrm{Ni}$, 
TABLE 9 - Continued

\begin{tabular}{|c|c|c|c|c|c|c|c|c|c|c|c|c|c|c|c|}
\hline $\begin{array}{l}\text { Core } \\
\text { Section } \\
\text { Interval (cm) }\end{array}$ & $\begin{array}{r}20 \\
1 \\
97\end{array}$ & $\begin{array}{r}21 \\
1 \\
19\end{array}$ & $\begin{array}{r}21 \\
4 \\
79\end{array}$ & $\begin{array}{r}22 \\
1 \\
29\end{array}$ & $\begin{array}{r}22 \\
2 \\
82\end{array}$ & $\begin{array}{r}22 \\
4 \\
26\end{array}$ & $\begin{array}{r}23 \\
2 \\
52\end{array}$ & $\begin{array}{r}23 \\
5 \\
73\end{array}$ & $\begin{array}{r}24 \\
1 \\
19\end{array}$ & $\begin{array}{r}24 \\
4 \\
73\end{array}$ & $\begin{array}{r}25 \\
2 \\
51\end{array}$ & $\begin{array}{r}26 \\
1 \\
33\end{array}$ & $\begin{array}{r}26 \\
3 \\
46\end{array}$ & $\begin{array}{r}27 \\
1 \\
112\end{array}$ & $\begin{array}{r}27 \\
2 \\
94\end{array}$ \\
\hline $\mathrm{SiO}_{2}(\%)$ & 47.8 & 47.1 & 50.9 & 46.3 & 47.1 & 46.1 & 50.5 & 50.5 & 46.4 & 48.4 & 51.0 & 48.1 & 49.6 & 45.9 & 46.1 \\
\hline $\mathrm{TiO}_{2}$ & 4.16 & 3.72 & 3.97 & 4.95 & 3.72 & 4.91 & 3.47 & 3.61 & 4.46 & 3.20 & 3.42 & 3.36 & 3.17 & 4.45 & 4.50 \\
\hline $\mathrm{Al}_{2} \overline{\mathrm{O}}_{3}$ & 13.1 & 13.4 & 13.2 & 13.7 & 14.9 & 13.1 & 13.4 & 13.6 & 13.7 & 13.5 & 13.1 & 14.4 & 13.3 & 13.2 & 13.5 \\
\hline $\mathrm{Fe}_{2} \mathrm{O}_{3}$ & 14.49 & 14.27 & 13.19 & 14.97 & 9.76 & 15.52 & 11.92 & 11.65 & 13.80 & 13.77 & 13.28 & 12.04 & 12.53 & 15.01 & 14.44 \\
\hline $\mathrm{MnO}$ & 0.21 & 0.18 & 0.16 & 0.23 & 0.24 & 0.21 & 0.17 & 0.14 & 0.45 & 0.25 & 0.15 & 0.25 & 0.23 & 0.26 & 0.22 \\
\hline $\mathrm{MgO}$ & 5.82 & 6.15 & 5.53 & 5.56 & 4.97 & 5.73 & 6.44 & 6.09 & 5.16 & 6.28 & 5.38 & 5.88 & 6.19 & 6.25 & 6.02 \\
\hline $\mathrm{CaO}$ & 10.16 & 10.16 & 8.92 & 8.30 & 11.53 & 11.08 & 9.24 & 9.43 & 11.10 & 10.42 & 9.02 & 10.80 & 10.86 & 9.49 & 9.57 \\
\hline $\mathrm{Na}_{2} \mathrm{O}$ & 2.50 & 2.39 & 2.72 & 3.07 & 2.97 & 2.44 & 2.74 & 2.83 & 2.34 & 2.41 & 2.61 & 2.77 & 2.56 & 2.51 & 2.48 \\
\hline $\mathrm{K}_{2} \mathrm{O}$ & 0.20 & 0.18 & 0.86 & 1.03 & 1.95 & 0.27 & 0.74 & 0.86 & 0.42 & 0.34 & 0.77 & 0.73 & 0.28 & 0.80 & 0.86 \\
\hline $\mathrm{P}_{2} \mathrm{O}_{5}$ & 0.43 & 0.39 & 0.42 & 0.51 & 0.35 & 0.48 & 0.40 & 0.41 & 0.54 & 0.38 & 0.40 & 0.39 & 0.37 & 0.47 & 0.46 \\
\hline L.o.i. & 0.94 & 1.49 & 0.54 & 1.11 & 2.03 & 0.86 & 1.14 & 1.17 & 0.66 & 0.56 & 0.50 & 1.19 & 1.09 & 1.40 & 1.57 \\
\hline Total & 99.86 & 99.47 & 100.36 & 99.70 & 99.45 & 100.67 & 100.15 & 100.26 & 98.95 & 99.50 & 99.60 & 99.81 & 100.08 & 99.72 & 99.74 \\
\hline $\mathrm{Ni}$ & 33 & 36 & 35 & 20 & 28 & 23 & 93 & 93 & 50 & 53 & 51 & 88 & 94 & 35 & 42 \\
\hline $\mathrm{Cr}$ & 23 & 28 & 27 & 3 & 30 & 12 & 176 & 174 & 64 & 83 & 50 & 169 & 175 & 32 & 36 \\
\hline $\mathrm{Zn}$ & 122 & 117 & 117 & 137 & 93 & 134 & 127 & 95 & 135 & 109 & 116 & 111 & 102 & 121 & 123 \\
\hline $\mathrm{Ga}$ & 27 & 27 & 24 & 28 & 22 & 26 & 27 & 27 & 23 & 24 & 26 & 28 & 21 & 24 & 23 \\
\hline $\mathrm{Rb}$ & 2 & 1 & 10 & 9 & 30 & $<1$ & 12 & 9 & 3 & 3 & 12 & 9 & 11 & 8 & 8 \\
\hline $\mathrm{Sr}$ & 447 & 439 & 430 & 453 & 756 & 443 & 413 & 409 & 452 & 415 & 393 & 423 & 426 & 431 & 442 \\
\hline $\mathrm{Y}$ & 37 & 35 & 37 & 41 & 28 & 40 & 37 & 36 & 38 & 35 & 37 & 35 & 33 & 42 & 41 \\
\hline $\mathrm{Zr}_{\mathrm{r}}$ & 302 & 274 & 290 & 378 & 211 & 299 & 268 & 273 & 341 & 251 & 255 & 243 & 229 & 312 & 307 \\
\hline $\mathrm{Nb}$ & 28 & 26 & 27 & 32 & 25 & 30 & 28 & 27 & 42 & 27 & 27 & 27 & 24 & 33 & 34 \\
\hline $\mathrm{Ba}$ & 143 & 105 & 174 & 225 & 332 & 131 & 169 & 151 & 252 & 129 & 179 & 165 & 102 & 193 & 206 \\
\hline $\mathrm{La}$ & 32 & 25 & 27 & 34 & 24 & 29 & 25 & 23 & 36 & 27 & 27 & 24 & 26 & 30 & 31 \\
\hline $\mathrm{Ce}$ & 60 & 60 & 65 & 70 & 48 & 69 & 54 & 58 & 70 & 58 & 60 & 54 & 50 & 68 & 68 \\
\hline $\mathrm{Pb}$ & $<1$ & 5 & 5 & 5 & 5 & $<1$ & 8 & 4 & 2 & 7 & 4 & 6 & 2 & 8 & 6 \\
\hline Th & 1 & 1 & 1 & 4 & $<1$ & 3 & 2 & $<1$ & 1 & 1 & 1 & 2 & $<1$ & 4 & $<1$ \\
\hline $\mathrm{Zr} / \mathrm{Nb}$ & 11 & 11 & 11 & 12 & 8 & 10 & 10 & 10 & 8 & 9 & 9 & 9 & 10 & 9 & 9 \\
\hline $\mathrm{Ti} / \mathrm{Zr}$ & 83 & 81 & 82 & 79 & 106 & 98 & 78 & 79 & 78 & 76 & 80 & 83 & 83 & 85 & 88 \\
\hline $\mathrm{Y} / \mathrm{Zr}$ & 0.12 & 0.13 & 0.13 & 0.11 & 0.13 & 0.13 & 0.14 & 0.13 & 0.11 & 0.14 & 0.15 & 0.14 & 0.14 & 0.13 & 0.13 \\
\hline $\mathrm{Ce} / \mathrm{Zr}$ & 0.20 & 0.22 & 0.22 & 0.19 & 0.23 & 0.23 & 0.20 & 0.21 & 0.21 & 0.23 & 0.24 & 0.22 & 0.22 & 0.22 & 0.22 \\
\hline $\mathrm{Ba} / \mathrm{Zr}$ & 0.47 & 0.38 & 0.60 & 0.60 & 1.57 & 0.44 & 0.63 & 0.55 & 0.74 & 0.51 & 0.70 & 0.68 & 0.45 & 0.62 & 0.67 \\
\hline $\mathrm{Ce}_{\mathrm{N}} / \mathrm{Y}_{\mathrm{N}}$ & 3.98 & 4.21 & 4.31 & 4.19 & 4.21 & 4.24 & 3.58 & 3.96 & 4.52 & 4.07 & 3.98 & 3.79 & 3.72 & 3.98 & 4.07 \\
\hline $\mathrm{Fe}_{\text {tot }} / \mathrm{Mg}$ & 2.89 & 2.69 & 2.77 & 3.12 & 2.28 & 3.14 & 2.15 & 2.22 & 3.10 & 2.54 & 2.86 & 2.37 & 2.35 & 2.78 & 2.78 \\
\hline $\mathrm{K} / \mathrm{R} \mathrm{b}$ & 822 & 1472 & 710 & 949 & 539 & - & 513 & 793 & 1154 & 936 & 530 & 674 & 209 & 829 & 889 \\
\hline $\mathrm{Ba} / \mathrm{Sr}$ & 0.32 & 0.24 & 0.40 & 0.50 & 0.44 & 0.30 & 0.41 & 0.37 & 0.56 & 0.31 & 0.46 & 0.39 & 0.24 & 0.45 & 0.47 \\
\hline Q & 2.4 & 1.4 & 4.6 & 0.0 & 0.0 & 0.0 & 3.1 & 2.8 & 1.2 & 1.4 & 5.3 & 0.0 & 2.5 & 0.0 & 0.0 \\
\hline Or & 1.2 & 1.1 & 5.0 & 6.1 & 11.6 & 1.6 & 4.4 & 5.1 & 2.5 & 2.0 & 4.5 & 4.3 & 1.6 & 4.7 & 5.1 \\
\hline $\mathrm{Ab}$ & 21.2 & 20.4 & 22.9 & 26.0 & 18.0 & 20.5 & 23.1 & 23.9 & 20.0 & 20.5 & 22.1 & 23.5 & 21.7 & 21.3 & 21.0 \\
\hline An & 24.0 & 25.4 & 21.2 & 20.6 & 21.6 & 23.8 & 22.0 & 21.8 & 25.8 & 25.1 & 21.8 & 24.6 & 23.8 & 22.5 & 23.3 \\
\hline $\mathrm{Ne}$ & 0.0 & 0.0 & 0.0 & 0.0 & 3.9 & 0.0 & 0.0 & 0.0 & 0.0 & 0.0 & 0.0 & 0.0 & 0.0 & 0.0 & 0.0 \\
\hline Di & 19.7 & 18.8 & 16.7 & 14.5 & 27.6 & 23.0 & 17.4 & 18.2 & 21.8 & 20.2 & 17.1 & 21.9 & 22.8 & 18.0 & 17.6 \\
\hline Hy & 18.0 & 19.7 & 17.1 & 11.2 & 0.0 & 15.6 & 18.2 & 16.3 & 14.5 & 19.6 & 17.7 & 11.1 & 16.4 & 14.0 & 15.2 \\
\hline O1 & 0.0 & 0.0 & 0.0 & 6.0 & 4.8 & 0.3 & 0.0 & 0.0 & 0.0 & 0.0 & 0.0 & 3.0 & 0.0 & 4.6 & 2.8 \\
\hline $\mathrm{Mt}$ & 2.5 & 2.5 & 2.3 & 2.6 & 1.7 & 2.7 & 2.1 & 2.0 & 2.4 & 2.4 & 2.3 & 2.1 & 2.2 & 2.6 & 2.5 \\
\hline II & 7.9 & $\begin{array}{l}7.1 \\
0.9\end{array}$ & 7.5 & 9.4 & 7.1 & 9.3 & 6.6 & 6.8 & 8.6 & 6.1 & 6.5 & 6.4 & 6.0 & 8.5 & 8.6 \\
\hline Ap & 1.0 & 0.9 & 1.0 & 1.2 & 0.8 & 1.1 & 0.9 & 1.0 & 1.3 & 0.9 & 0.9 & 0.9 & 0.9 & 1.1 & 1.1 \\
\hline
\end{tabular}

aNormative values based on $\mathrm{Fe}_{2} \mathrm{O}_{3} / \mathrm{FeO}$ ratio of 0.15 . $\mathrm{Fe}_{2} \mathrm{O}_{3}$ is total iron as $\mathrm{Fe}_{2} \mathrm{O}_{3}$. $\mathrm{Ce} / \mathrm{Y}_{\mathrm{N}}$ represents ratio of chondrite-normalized values.

and $\mathrm{Cr}$ invariably contain cumulus olivine, pyroxene, or chrome spinel, probably resulting from within-unit (within-flow or within-sill) crystal settling. This is especially noticeable in geochemical units 442-1, 443-3, 444-2, and 446-5. Within-sill or within-flow differentiation may play an important role in determining the observed within-unit chemical variation, but it is often difficult to ascertain whether pre-eruptive differentiation, followed by contemporaneous emplacement of basalts of slightly different compositions as single flow or sill units, is an important factor. Within-sill or within-flow differentiation may be caused by gravitational settling of mineral phases with a high specific gravity, or crystal sorting during magma flow through the sill (or flow) body. Detailed studies of individual sills and flows (Marsh, in prep.) suggests that in situ crystal cumulation plays an important role.
Compositional variation between individual lithologic and geochemical units is probably caused by pre-eruptive differentiation, i.e., fractional crystallization and (or) partial melting. However, proving consanguinity of magmas at the same site is difficult, because of uncertainty surrounding the time between successive magma batches. Thus, at Site 442 for example, geochemical units $442-2,3$, and 4 cannot be directly related to unit 1 because of the 2-m.y. interval separating the two groups. However, the four units at Site 442 appear to be essentially comagmatic, indicating derivation from a compositionally identical mantle source, although distinct partial-melting episodes rather than magma batches from a single fractionating magma chamber are involved. Similarly, the basalts from Site 443 appear to have been derived from a single mantle source, although, unlike the units of Site 442 , the less-HYG/more-HYG 


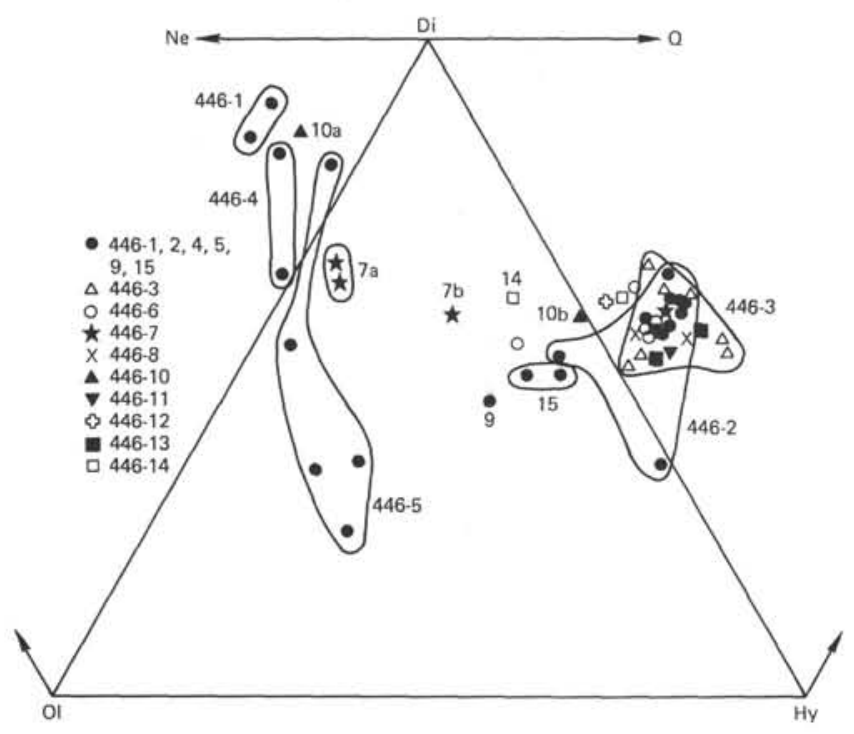

Figure 37. Normative nepheline (Ne), olivine $(\mathrm{Ol})$, diopside (Di), Hypersthene (Hy), quartz (Q) diagram for Site 446. Normative values based on $\mathrm{Fe}_{2} \mathrm{O}_{3} / \mathrm{FeO}$ ratio of 0.15 .

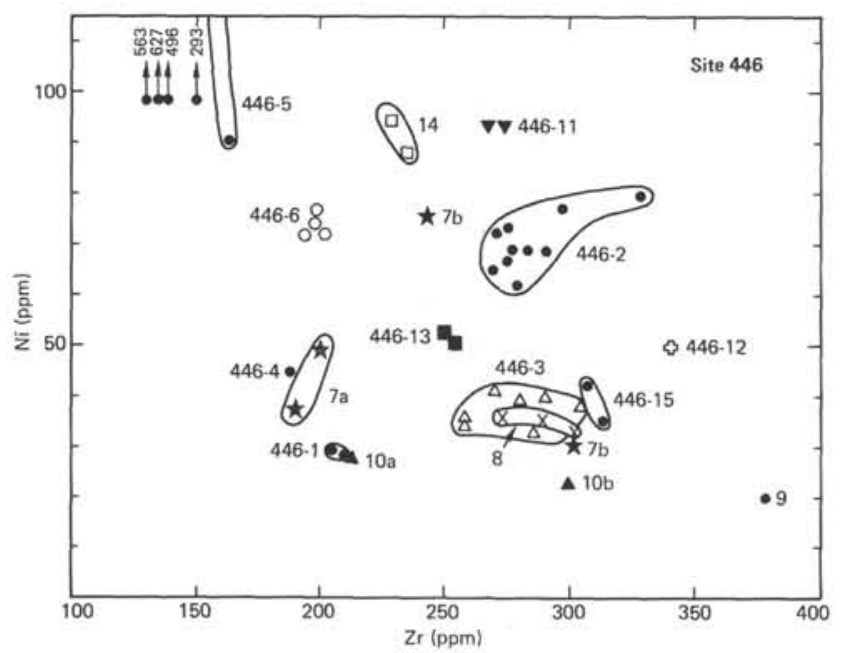

Figure 38. Ni versus Zr, Site 446. Separate geochemical units are labelled. Symbols as in Figure 37.

element ratios (e.g., $\mathrm{Y} / \mathrm{Zr}, \mathrm{Th} / \mathrm{Hf}$ ) show slight variation among the various units (e.g., Figure 20; Wood et al., this volume). It is considered unlikely that closed-system fractional crystallization (Neumann et al., 1954) of a basaltic magma could produce significant changes in less-HYG/more-HYG ratios, unless the degree of fractionation becomes unreasonably high. Batch partial melting may, however, change these ratios (e.g., data on Leg 49 basalts in Tarney et al., 1978), so it is reasonable to assume that the various units of Site 443 are related through different degrees of partial melting of the same source. In addition, the compositional similarity of the Site 442 and 443 basalts suggests that the sources from which the basalts were derived are very similar. This is not unexpected in view of the fact that both sites are

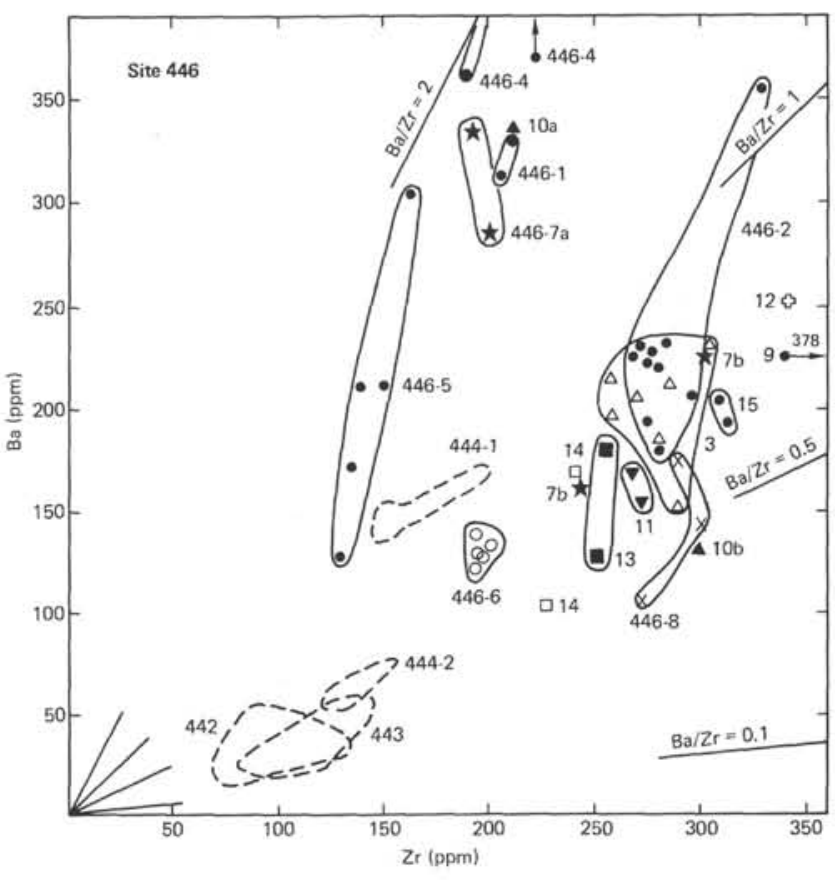

Figure 39. Ba versus $\mathrm{Zr}$, Site 446. Field for Site 442 from Figure 13; for Site 443 from Figure 24; and for Site 444 from Table 5.

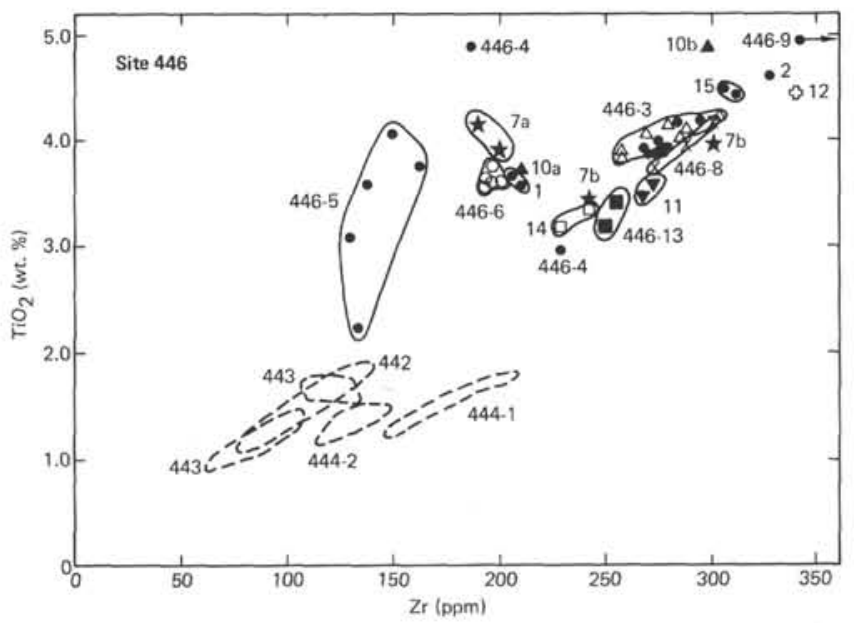

Figure 40. $\mathrm{TiO}_{2}$ versus $\mathrm{Zr}$, Site 446. Field for Site 442 from Figure 7; for Site 443 from Figure 19; for Site 444 from Figure 32.

located on the same mantle flow line on opposite sides of the spreading axis.

Inter-unit variation at Sites 444 and 446 is less easily explained. Off-ridge basaltic material was penetrated at both sites, and the basalts at both sites are compositionally heterogenous and distinct from the Site 442 and 443 basalts. The within-unit variations at Site 444 may be adequately explained by fractional crystallization in sub-axis magma chambers, and perhaps also in individual flow units. However, the inter-unit variation cannot be explained by these processes, because of the wide disparity in less-HYG/more-HYG element ratios (e.g., 


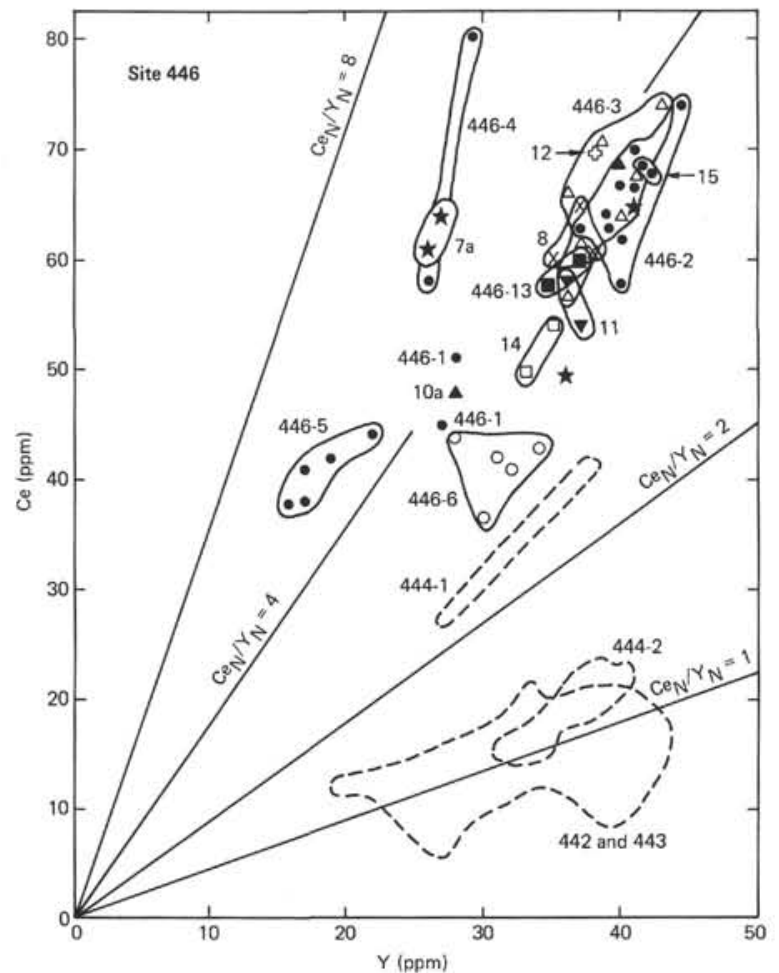

Figure 41. Ce versus $Y$, Site 446. Field for Site 442 from Figure 16; for Site 443 from Figure 25; for Site 444 from Figure 35.

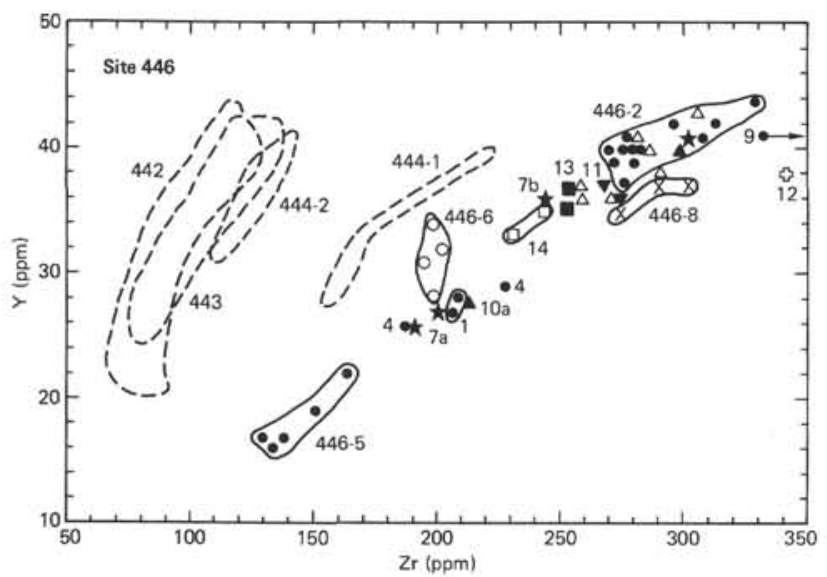

Figure 42. $Y$ versus $\mathrm{Zr}$, Site 446. Fields for Sites 442, 443, and 444 from Figure 33.

$\mathrm{Y} / \mathrm{Zr}, \mathrm{TiO}_{2} / \mathrm{Zr}$ ) and more-HYG element ratios (e.g., $\mathrm{Ce} / \mathrm{Zr}, \mathrm{Zr} / \mathrm{Nb}$ ). More important, however, is that the more-HYG-element ratios cannot be changed during the partial melting processes which may be expected during the formation of basaltic magmas (Tarney et al., 1979; Wood et al., 1979; Bougault et al., 1979). Detailed studies of basalts from the North Atlantic Ocean have shown that variations in more-HYG-element ratios (Bougault et al., 1979; Tarney et al., 1979; Wood et al., 1979) and variations in $\mathrm{Sr}-, \mathrm{Pb}-$, and $\mathrm{Nd}$-isotope ratios

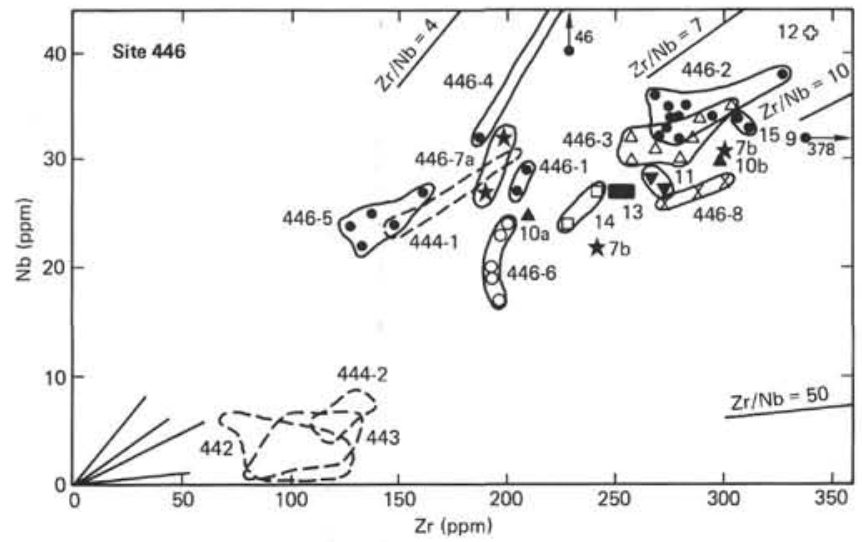

Figure 43. Nb versus $\mathrm{Zr}$, Site 446. Fields for Sites 442, 443, and 444 from Figures 9 and 31 .

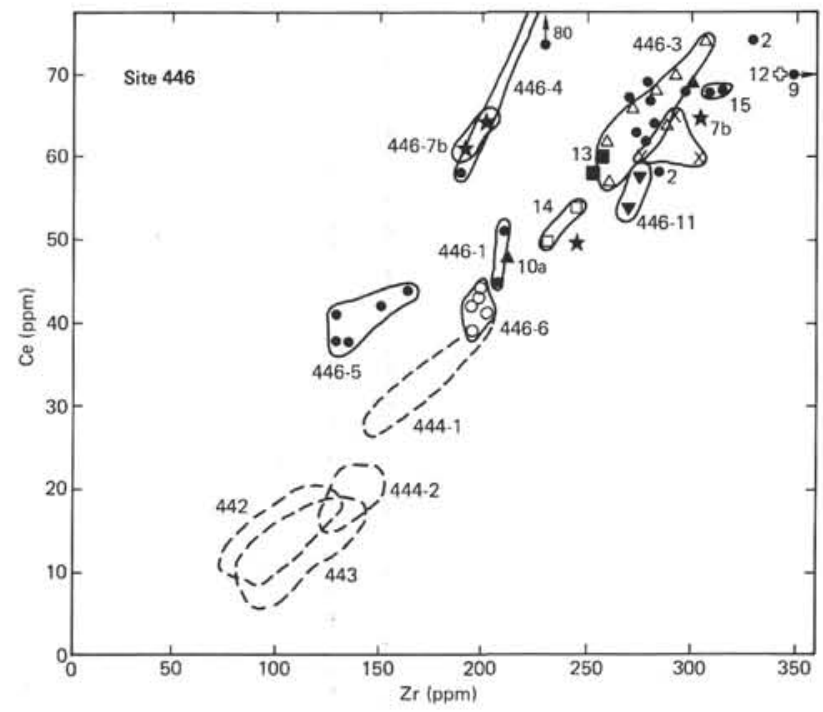

Figure 44. Ce versus $\mathrm{Zr}$, Site 446. Fields for Sites 442, 443, and 444 from Figures 8 and 26 and Table 5, respectively.

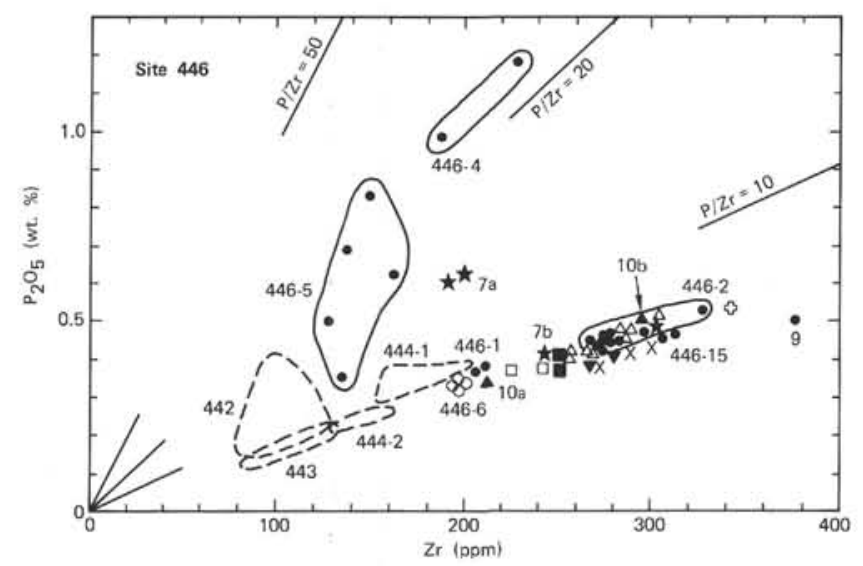

Figure 45. $P_{2} O_{5}$ versus $\mathrm{Zr}$, Site 446. Fields for Sites 442, 443, and 444 from Figures 10 and 21 and Table 5, respectively. 
(e.g., O'Nions et al., 1977; Sun et al., 1979) are more likely to have been caused by chemical heterogeneities in the mantle source (e.g., Tarney et al., 1979). The nature and causes of these heterogeneities are still in dispute (Schilling, 1975a,b; Hart et al., 1973; White et al., 1976; Tarney et al., 1979, in press; Wood et al., 1979, in press), but they appear to have arisen during successive episodes of crustal extraction and mantle metasomatism (e.g., Lloyd and Bailey, 1975; Frey et al., 1978; Pearce and Norry, 1979; Tarney et al., in press). In the Shikoku Basin, and possibly also the Daito Basin, there is the added possibility and complexity of a chemical component being derived from an adjacent subduction zone.

Detailed modeling is required before partial melting can be ruled out as the dominant process governing the HYG-element distributions in the Site 444 and 446 basalts, and the variations attributed solely to mantle heterogeneity. The available data do suggest, however, that the various units at each of the two sites were derived from compositionally varied sources. The basalts of unit $444-1$ and Site 446 are chemically similar to evolved E-type basalts commonly found on oceanic islands (e.g., Iceland; Wood et al., in press), where such basalts occur in conjunction with T-type tholeiites: the mantle source of the E-type basalts characteristically yields a mixture of enriched and depleted basalts in any one area.

Representative analyses of the Shikoku Basin basalts, and of the basalts from the East Scotia Sea and Bransfield Strait back-arc basins, are given in Table 5; typical analyses of N-, T-, and E-type basalts from the Atlantic Ocean are included in Table 4. Because of the effects of partial melting, fractional crystallization, and withinflow or within-sill differentiation, it is more valid to consider HYG-element ratios, rather than absolute element abundances, when comparing basalt compositions from different tectonic environments. In particular, ratios involving the more-HYG elements which in ocean-ridge basalts are generally considered to include $\mathrm{La}, \mathrm{Ce}, \mathrm{Nb}, \mathrm{Ba}, \mathrm{K}, \mathrm{Rb}$, and $\mathrm{Zr}$, are less responsive to fractionation processes, as mentioned before (see Wood et al., 1979).

The basalts from Sites 442 and 443, and unit 444-2, are chemically similar to basalts recovered by dredges 20 and 23 (e.g., D20.43B, Table 5) in the East Scotia Sea (Saunders and Tarney, 1979). Although they tend in composition toward $\mathrm{N}$-type (depleted) ocean-ridge basalts, having high $\mathrm{Zr} / \mathrm{Nb}$ ratios and low $\mathrm{Ce} / \mathrm{Y}, \mathrm{Ce} / \mathrm{Zr}$, and $\mathrm{P} / \mathrm{Zr}$ ratios, they nonetheless have higher $\mathrm{Ce} / \mathrm{Y}$, $\mathrm{Ba} / \mathrm{Zr}, \mathrm{Ba} / \mathrm{Sr}$ ratios, and probably higher $\mathrm{K} / \mathrm{Zr}$ and $\mathrm{Rb} / \mathrm{Zr}$ ratios, than $\mathrm{N}$-type ocean-ridge basalts. Equally, they have higher $\mathrm{Zr} / \mathrm{Nb}$ and lower $\mathrm{Ce} / \mathrm{Y}$ and $\mathrm{Ce} / \mathrm{Zr}$ ratios than transitional (T-type) basalts, for example those from $63^{\circ} \mathrm{N}$ on the Reykjanes Ridge (Tarney et al., 1979; Wood et al., 1979).

The transitional island-arc-tholeiite characteristics exhibited by the basalts from dredge 24 in the East Scotia Sea (e.g., D24.14; Table 5) (high $\mathrm{SiO}_{2}$, high $\mathrm{K} / \mathrm{Zr}$ and $\mathrm{Ba} / \mathrm{Zr}$ ratios, and low $\mathrm{Ni}$ and $\mathrm{Cr}$ contents) are not observed in any of the Leg 58 basalts. Island-arc tholeiites and calc-alkaline basalts typically have low absolute abundances of the high-field-strength (HFS) elements ( $\mathrm{Ti}, \mathrm{P}, \mathrm{Zr}, \mathrm{Hf}, \mathrm{Nb}, \mathrm{Ta}$ ), and higher absolute abundances of the large-ion lithophile (LIL) elements $(\mathrm{K}, \mathrm{Rb}$, $\mathrm{Ba}, \mathrm{Sr}, \mathrm{Th}, \mathrm{U}, \mathrm{Ce}$, and $\mathrm{La}$ ) (Saunders et al., in press). Although some of the Site 444 basalts and all the Site 446 basalts exhibit enrichment in the LIL elements, they also show significant enrichment in the HFS elements, a correlation more characteristic of alkaline basalts than orogenic basalts.

Lavas from Bransfield Strait, a small, young, intracontinental, back-arc basin at the north end of the Antarctic Peninsula, exhibit chemistries which may be considered transitional between ocean-ridge and calc-alkaline basalts (Weaver et al., 1979). Some of the lavas have significantly higher levels of $\mathrm{K}$ and $\mathrm{Rb}$ (Bridgeman Island) and $\mathrm{Ba}$ and $\mathrm{Sr}$, and higher $\mathrm{Ce}_{\mathrm{N}} / \mathrm{Y}_{\mathrm{N}}$ ratios than the Shikoku Basin basalts (with the exception of geochemical unit 444-1). Apart from their vesicularity, however, basalts recovered from the Shikoku Basin cannot be distinguished from basalts being erupted along some sections of ocean ridges in the major ocean basins. They do not show the extreme depletion in certain HYG elements $(\mathrm{K}, \mathrm{Rb}, \mathrm{Ba}$, light $\mathrm{REE}, \mathrm{Nb})$ typical of $\mathrm{N}$-type basalts, and may be best considered as intermediate between $\mathrm{N}$ - and T-type ocean-ridge basalts. Whereas they do not show any chemical characteristics transitional toward orogenic basalts, their high vesicularity does suggest that their mantle sources may have been enriched in volatiles, possibly derived from the adjacent subduction zone.

The HYG-element-enriched alkaline and tholeiitic basalts from Site 446 and from unit 444-1 more closely resemble enriched E-type basalts recovered from ocean islands and so-called "hot-spot" regions (e.g., $45^{\circ} \mathrm{N}$ ) in the major ocean basins (Table 4). However, there are some significant differences, both between the 444-1 and 446 basalts and between these and E-type basalts. For example, the basalts from Site 446 have much higher levels of $\mathrm{TiO}_{2}$ and total iron than basalts recovered from $45^{\circ} \mathrm{N}$ on the Mid-Atlantic Ridge; they more closely resemble HYG-element-enriched ferrobasalts commonly found on the oceanic islands (Table 4). In addition, the basalts of unit 444-1 have very high $\mathrm{K} / \mathrm{Rb}$ ratios, a feature atypical of E-type basalts and their differentiates; nonetheless they have low $\mathrm{Zr} / \mathrm{Nb}$ ratios and high $\mathrm{Ba} / \mathrm{Zr}, \mathrm{Ce} / \mathrm{Y}$, and $\mathrm{Ce} / \mathrm{Zr}$ ratios, similar to the Site 446 basalts.

It appears that the compositional range of basalts recovered from the Shikoku and Daito Basins is as great as for those being erupted at major oceanic spreading centers. Because the trace-element characteristics of basalts reflect, to a first approximation, those of their mantle source regions, it may be assumed that an equal diversity of mantle sources is available in the marginalbasin environment. This attains significance if "enriched" mantle sources are attributed to the influence of a deep mantle plume (e.g., Schilling, 1973), because, with the close association between marginal basins and subduction zones, there is an inherent tectonic problem in circumventing the subducted slab. This problem may be overcome if, for example, the "enriched" mantle 
overlies the N-type basalt mantle source, or if the different mantle types are intimately associated by a veining process, as suggested by Tarney et al. (in press). In addition, the marginal-basin mantle source may contain a component from the subducted slab (Saunders and Tarney, 1979). On the basis of the available chemical data, this does not appear to be the case for the Shikoku Basin samples. However, their high vesicularity may reflect a high volatile content in the mantle source, possibly due to a hydrous component arising from the subducted slab.

\section{ACKNOWLEDGMENTS}

We would like to thank Dr. G. L. Hendry for providing analytical facilities, and for assistance in drafting the section on analytical techniques. We also thank Nigel C. B. Donnellan for drawing many of the text figures, and the DSDP technicians for their assistance in sample and thin-section preparation. N. G. M. and A. D. S. would like to acknowledge the Natural Environment Research Council, U. K., for providing financial support during the tenure of this project.

\section{REFERENCES}

Barker, P. F., 1972. A spreading centre in the East Scotia Sea. Earth Planet. Sci. Lett., 15, 123-132.

Bass, M. N., Moberly, R., Rhodes, J. M.,Shih, C. Y., and Church, S. E., 1973. Volcanic rocks cored in the Central Pacific, Leg 17, Deep Sea Drilling Project. In Winterer, E. L., Ewing, J. I., et al., Init. Repts. DSDP, 34: Washington (U. S. Govt. Printing Office), pp. 429-446.

Bennett, H., and Oliver, G. J., 1976. Development of fluxes for the analysis of ceramic materials by X-ray fluorescence spectrometry. Analyst, 101, 803-807.

Bougault, H., Cambon, P., Corre, O., Treuil, M., and Joron, J.-L., 1979. Evidence for variability of magmatic processes and upper mantle heterogeneity in the axial region of the Mid-Atlantic Ridge near $22^{\circ} \mathrm{N}$ and $36^{\circ} \mathrm{N}$. Tectonophysics, $55,11-34$.

Cann, J. R., 1970. Rb, Sr, Y, Zr and $\mathrm{Nb}$ in some ocean floor basaltic rocks. Earth Planet. Sci. Lett., 10, 7-11.

Dewey, J. F., 1976. Ophiolite obduction. Tectonophysics. 31, 93-120.

Engel, A. E., Engel, C. G., and Havens, R. G., 1965. Chemical characteristics of oceanic basalts and the upper mantle. Geol. Soc. Am. Bull., 76, 719-734.

Erlank, A. J., and Kable, E. J. D., 1976. The significance of incompatible elements in Mid-Atlantic Ridge basalts from $45^{\circ} \mathrm{N}$ with particular reference to $\mathrm{Zr} / \mathrm{Nb}$. Contr. Mineral. Petrol., 54, 281-291.

Frey, F. A., Bryan, W. B., and Thompson, G., 1974. Atlantic Ocean floor: geochemistry and petrology of basalts from Legs 2 and 3 of the Deep Sea Drilling Project. J. Geophys. Res., 79, 5507-5527.

Frey, F. A., Green, D. H., and Roy, S. D., 1978. Integrated models of basalt petrogenesis. J. Petrol., 19, 463-513.

Garcia, M. O., Liu, N. W. K., and Meunow, D. W., 1979. Volatiles in submarine volcanic rocks from the Mariana Island arc and trough. Geochim. Cosmochim. Acta, 43, 305-312.

Green, D. H., 1970. The origin of basaltic and nephelenitic magmas. Trans. Leicester Phil. Soc., 64, 26-54.

Hart, S. R., 1971. K, Rb, Cs, Sr and Ba contents and Sr-isotope ratios of ocean floor basalts. Phil. Trans. Roy. Soc. London, A268, 573-588.
Hart, S. R., Glassley, W. E., and Karig, D. E., 1972. Basalts and seafloor spreading behind the Mariana Island Arc. Earth Planet. Sci. Lett., 15, 12-18.

Hart, S. R., Erlank, A. J., and Kable, E. J. D., 1974. Sea floor basalt alteration: some chemical and strontium isotopic effects. Contr. Mineral. Petrol., 44, 219-230.

Hart, S. R., Schilling, J.-G., and Powell, J. L., 1973. Basalts from Iceland and along the Reykjanes Ridge: Sr-isotope geochemistry. Nature, 246, 104-107.

Harvey, P. K., Taylor, D. M., Hendry, G. L., and Bancroft, F., 1973. An accurate fusion method for the analysis of rocks and chemically related materials by X-ray fluorescence spectrometry. X-Ray Spectrom., 2, 33-44.

Hawkins, J. W., 1974. Geology of the Lau Basin, a marginal basin behind the Tonga arc. In Burke, C. A. and Drake, C. L. (Eds.) The Geology of Continental Margins: New York (Springer-Verlag), 505-520. 1976. Petrology and geochemistry of basaltic rocks of the Lau Basin. Earth Planet. Sci. Lett., 28, 283-297.

1977. Petrological and geochemical characteristics of marginal basin basalts. In Talwani, M., and Pitman, W. C. III,(Eds.), Island Arcs, Deep Sea Trenches and BackArc Basins: Washington (Amer. Geophys. Union), 355365 .

Hendry, G. L., 1975. X-ray fluorescence. In Nicol, A. W. (Ed.), Physicochemical methods of mineral analysis: New York (Plenum Press), pp. 87-152.

Jones, J. G., 1969. Pillow lavas as depth indicators. Am. J. Sci., 267, 181-195.

Karig, D. E., 1971. Origin and development of marginal basins in the western Pacific. J. Geophys. Res., 76, 2542-2561.

1974. Evolution of arc systems in the western Pacific. In Annual Review of Earth and Planetary Sciences, 2, 51-75.

1975. Basin genesis in the Philippine Sea. In Karig, D. E. and Ingle, J. E., et al., Init. Repts. DSDP, 31: Washington (U. S. Govt. Printing Office), pp. 857-879.

Karig, D. E., Ingle, J. E., et al., 1975. Init. Repts. DSDP, 31: Washington (U. S. Govt. Printing Office).

Katsumata, M., and Sykes, L. R., 1969. Seismicity and tectonics of the western Pacific: Izu-Mariana-Caroline and Ryukyu-Taiwan region. J. Geophys. Res., 74, 5923-5948.

Kay, R. W., Hubbard, J. J., and Gast, P. W., 1970. Chemical characteristics and origin of oceanic ridge volcanic rocks. $J$. Geophys. Res., 75, 1585-1613.

Kobayashi, K., and Isezaki, N., 1976. Magnetic Anomalies in the Sea of Japan and the Shikoku Basin: Possible tectonic implications. In Sutton, G. H., Manghani, M. H., Moberly, R. (Eds.), The Geophysics of the Pacific Ocean Basin and Its Margin. Am. Geophys. Union Monogr., 19.

Kobayashi, K., and Nakata, M., 1977. Local magnetic anomaly profiles, Shikoku Basin, Northwestern Pacific Ocean. Contr. Geodynamics Project Japan, 77-2.

LaBrecque, J. L., Kent, D. V., and Cande, S. C., 1977. Revised magnetic polarity time scale for Late Cretaceous and Cenozoic time. Geology, 5, 330-335.

Leake, B. E., Hendry, G. L., Kemp, A., Plant, A. G., Harvey, P. K., Wilson, J. R., Coats, J. S., Aucott, J. W., Lunel, T., and Howarth, R. J., 1969. The chemical analysis of rock powders by automatic X-ray fluorescence. Chem. Geol., 5, 7-86.

Lloyd, F. E., and Bailey, P. K., 1975. Light element metasomatism of the continental mantle: the evidence and the consequences. In Ahrens, L. H., Press, F., Runcorn, S. K. and Urey, H. C. (Eds.), Physics and Chemistry of the Earth, (Vol. 9): New York (Pergamon Press), pp. 389-416. 
Ludden, J. N., and Thompson, G., 1979. An evaluation of the behaviour of the rare earth elements during the weathering of sea floor basalt. Earth. Planet. Sci. Lett., 43, 85-92.

Meunow, D. W., Lui, N. W. K., Garcia, M. O., and Saunders, A. D., in press. Volatiles in submarine volcanic rocks from the spreading axis of the East Scotia Sea back-arc basin. Earth Planet., Sci. Lett.

Mitchell, W. S., and Aumento, F., 1977. Uranium in oceanic rocks: Deep Sea Drilling Project Leg 37. Can. J. Earth Sci., 14, 794-808.

Mizuno, A., Okuda, Y., Tamaki, K., Kinoshita, Y., Nohara, M., Yuasa, M., Nakajima, M., Murakami, F., Terashima, S., and Ishibashi, K., 1975. Marine geology and geological history of the Daito Ridge area, northwestern Philippine Sea. Marine Sci., 7, 484-491, 543-548.

Mizuno, A., Okuda, Y., Nagumo, S., Kagami, H., and Nasu, N., in press. Subsidence of the Daito Ridge and associated basins, North Philippine Sea.

Moore, J. G., 1965. Petrology of deep-sea basalt near Hawaii. Am. J. Sci., 263, 40-52.

Murauchi, S., Asanuma, T. and Saki, K., 1976. Seafloor spreading in the Shikoku Basin, South of Japan. Abstr. Progr., Int. Ewing Symp., Harriman, New York.

Murauchi, S., Den, N., Asano, S., Hotta, H., Yoshii, T., Asanuma, T., Hagiwara, K., Ichikawa, K., Sata, R., Ludwig, W. J., Ewing, J. I., Edgar, N. T., and Houtz, R. E., 1968. Crustal structure of the Philippine Sea. J. Geophys. Res., 73, 3143-3171.

Neumann, H., Mead, J., and Vitaliano, C. J., 1954. Trace element variation during fractional crystallization as calculated from the distribution law. Geochim. Cosmochim. Ac$t a, 6,90-100$.

O'Hara, M. J., 1973. Non-primary magmas and the dubious mantle plume beneath Iceland. Nature, 243, 507-508. 1977. Geochemical evolution during fractional crystallization of a periodically refilled magma chamber. $\mathrm{Na}$ ture, 226, 503-507.

O'Nions, R. K., Hamilton, P. J., and Evensen, N. M., 1977. Variations in ${ }^{143} \mathrm{Nd} /{ }^{144} \mathrm{Nd}$ and ${ }^{87} \mathrm{Sr} / 86 \mathrm{Sr}$ ratios in oceanic basalts. Earth Planet. Sci. Lett., 34, 13-22.

O'Nions, R. K., Pankurst, R. J., and Gronvold, K., 1976. Nature and development of basalt magma sources beneath Iceland and the Reykjanes Ridge. J. Petrol., 17, 3-30.

Pearce, J. A., 1975. Basalt geocemistry used to investigate past tectonic environments on Cyprus. Tectonophysics, 25, 4167.

Pearce, J. A., and Cann, J. R., 1971. Ophiolite origin investigated by discriminant analysis using $\mathrm{Ti}, \mathrm{Zr}$ and $\mathrm{Y}$. Earth Planet. Sci. Lett., 12, 339-349.

1973. Tectonic setting of basic volcanic rocks using trace element analyses. Earth Planet. Sci. Lett., 19, 290300.

Pearce, J. A., and Norry, M. J., 1979. Petrologic implications of $\mathrm{Ti}, \mathrm{Zr}, \mathrm{Y}$ and $\mathrm{Nb}$ variations in volcanic rocks. Contrib. Mineral. Petrol., 57.

Reynolds, R. C., 1967. Estimation of mass absorption coefficients by Compton scattering: improvements and extensions of the method. Am. Mineral., 52, 1493-1502.

Rhodes, J. M., Blanchard, D. P., Rodgers, K. V., Jacobs, J. W., and Brannon, J. C., 1976. Petrology and chemistry of basalts from the Nazca Plate: Part 2-Major and trace element chemistry. In Hart, S. R., Yeats, R. S., et al., Init. Repts. DSDP, 34: Washington (U. S. Govt. Printing Office), pp. 239-244.

Saunders, A. D., and Tarney, J., 1979. The geochemistry of basalts from a back-arc spreading centre in the East Scotia Sea. Geochim. Cosmochim. Acta, 43, 555-572.
Saunders, A. D., Tarney, J., Stern, C., and Dalziel, I. W. D., 1979. Geochemistry of Mesozoic marginal basin floor igneous rocks from southern Chile. Geol. Soc. Amer. Bull., 90, 237-258.

Saunders, A. D., Tarney, J., and Weaver, S. D., in press. Transverse variations in chemistry across the Antartic Peninsula: implications for calc-alkaline magma genesis. Earth Planet. Sci. Lett.

Schilling, J.-G., 1971. Seafloor evolution: rare earth evidence. Phil. Trans. Roy. Soc. Lond., A268, 663-706. 1973. Iceland mantle plume: geochemical study of Reykjanes Ridge. Nature, 242, 565-575.

1975a. Azores mantle blob: rare earth evidence. Earth Planet. Sci. Lett., 25, 103-115.

1975 b. Rare earth variations across "normal segments" of the Reykjanes Ridge, 60 N-53 N, Mid-Atlantic Ridge, 29 S, and East Pacific Rise, 2 S-19 S, and evidence on the composition of the Low-velocity layer. J. Geophys. Res., 80, 1459-1473.

Shido, F., Miyashiro, A., and Ewing, M., 1971. Crystallization of abyssal tholeiites. Contr. Mineral. Petrol., 31, 251-266.

Sun, S.-S., Nesbitt, R. W., and Sharaskin, A. Ya., 1979. Chemical characteristics of mid-ocean ridge basalts. Earth Planet. Sci. Lett., 29.

Tarney, J., Saunders, A. D., Weaver, S. D., Donnellan, N. C. B., and Hendry, G. L., 1978. Minor element geochemistry of basalts from Leg 49, North Atlantic Ocean. In Luyendyk, B. P., Cann, J. R., et al., Init. Repts. DSDP, 49: Washington (U. S. Govt. Printing Office), pp. 657-691.

Tarney, J., Wood, D. A., Saunders, A. D., Varet, J., and Cann, J. R., 1979. Nature of mantle heterogeneity in the North Atlantic: evidence from Leg 49. In Talwani, M. (Ed.), Results of Deep Sea Drilling in the Atlantic. Maurice Ewing Series 2 (Amer. Geophys. Union).

Tarney, J., Wood, D. A., Saunders, A. D., and Cann, J. R., in press. Nature of mantle heterogeneity in the North Atlantic: evidence from deep sea drilling. Phil. Trans. Roy. Soc. London.

Tomada, Y., Kobayashi, K., Segawa, J., Nomura, M., Kimura, K., and Saki, T., 1975. Linear magnetic anomalies in the Shikoku Basin, northeastern Philippine Sea. J. Geomagnet. Geoelec., 28, 47-56.

Watts, A. B., and Weissel, J. K., 1975. Tectonic history of the Shikoku marginal basin. Earth Planet. Sci. Lett., 25, 239-250.

Watanabe, T., Epp, D., Uyeda, S., Langseth, M., and Yasui, M., 1970. Heat flow in the Philippine Sea. Tectonophysics, $10,205-224$.

Weaver, S. D., Saunders, A. D., Pankhurst, R. J. and Tarney, J., 1979. A geochemical study of magmatism associated with the initial stages of back-arc spreading. The quaternary volcanics of Bransfield Strait, from South Shetland Islands. Contr. Mineral. Petrol., 68, 151-169.

Weaver, S. D., Sceal, J. S. C., and Gibson, I. L., 1972. Traceelement data relevant to the origin of trachytic and pantelleritic lavas in the East African Rift system. Contr. Mineral. Petrol., 36, 181-194.

White, W. M., and Schilling, J.-G., 1978. The nature and origin of geochemical variations in Mid Atlantic Ridge basalts from the central North Atlantic. Geochim. Cosmochim. Acta, 42, 1501-1516.

White, W. M., Schilling, J.-G. and Hart, S. R., 1976. Evidences for the Azores mantle plume from strontium isotope geochemistry of the Central North Atlantic. Nature, 263, 659-663. 
Wood, D. A., Tarney, J., Varet, J., Saunders, A. D., Bougault, H., Joron, J.-L., Treuil, M., and Cann, J. R., 1979. Geochemistry of basalts drilled in the North Atlantic by IPOD Leg 49: implications for mantle heterogeneity. Earth Planet. Sci. Lett., 42, 77-97.
Wood, D. A., Joron, J.-L., Treuil, M., and Tarney, J., in press. Elemental and $\mathrm{Sr}$ isotope variations in basic lavas from Iceland and the surrounding ocean floor: the nature of mantle source inhomogeneities. Contr. Mineral. Petrol. 